NBSIR 80-2032

\title{
Kinetic and Photochemical Data for Atmospheric Chemistry Reactions of the Nitrogen Oxides
}

R. F. Hampson, Jr.

Center for Thermodynamics and Molecular Science

National Bureau of Standards

U.S. Department of Commerce

Washington, DC 20234

Jan. - Dec. 1979

Final

Issued May 1980

Prepared for

Federal Aviation Administration, Washington, DC 20591

NACA Washington, DC 20546 and

$-Q C=$ Standard Reference Data, NBS, Washington, DC 20234

100

.456

No. $80-2032$

1979

c. 2 
KINETIC AND PHOTOCHEMICAL

DATA FOR ATMOSPHERIC CHEMISTRY REACTIONS OF THE NITROGEN OXIDES

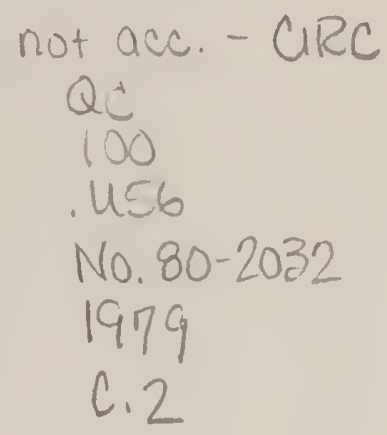

R. F. Hampson, Jr.

Center for Thermodynamics and Molecular Science National Bureau of Standards

U.S. Department of Commerce

Washington, DC 20234

Jan. - Dec. 1979

Final

Issued May 1980

Prepared for

Federal Aviation Administration, Washington, DC 20591 NASA, Washington, DC 20546 and

Office of Standard Reference Data, NBS, Washington, DC 20234

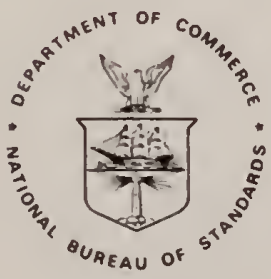

U.S. DEPARTMENT OF COMMERCE, Philip M. Klutznick, Secretary Luther H. Hodges, Jr., Deputy Secretary Jordan J. Baruch, Assistant Secretary for Productivity. Technology, and Innovation NATIONAL BUREAU OF STANDARDS, Ernest Ambler, Director 
1 


$$
\begin{aligned}
& \text { Kinetic and Photochemical Data for } \\
& \text { Atmospheric Chemistry Reactions of the Nitrogen Oxides }
\end{aligned}
$$

R. F. Hampson

\author{
Cherical Kinetics Division \\ National Measurement Laboratory \\ National Bureau of Standards \\ Washington, DC 20234
}

\title{
Prepared for
}

Federal Aviation Administration, Washington, DC 20591, NASA Washington, DC 20546 and Office of Standard Reference Data, NBS, Washington, DC 20234. 
Abstract . . . . . . . . . . . . 1

1. Introduction............ . 2

2. Guide to the Data Sheets ......... 2

2.1 Thermal Reactions . . . . . . . . 2

2.2 Photochemical Reactions....... 4

2.3 Assignment of Errors . . . . . . . 4

3. Data Sheets

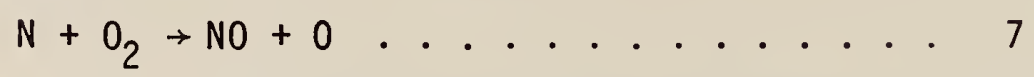

$\mathrm{N}+\mathrm{O}_{3} \rightarrow \mathrm{NO}+\mathrm{O}_{2} \cdot . . . . . . . . . .10$

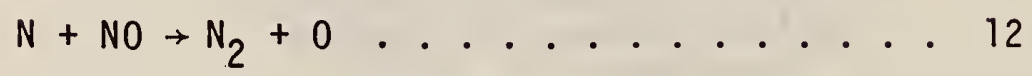

$\mathrm{N}+\mathrm{NO}_{2} \rightarrow$ products . . . . . . . . . . 14

$\mathrm{O}+\mathrm{NO}_{2} \rightarrow \mathrm{NO}+\mathrm{O}_{2}$. . . . . . . . . . 17

$\mathrm{O}+\mathrm{NO}_{3} \rightarrow \mathrm{O}_{2}+\mathrm{NO}_{2}$............ 20

$\mathrm{O}+\mathrm{N}_{2} \mathrm{O}_{5} \rightarrow$ products . . . . . . . . . 22

$O\left({ }^{1} D\right)+O_{2} \rightarrow O\left({ }^{3} P\right)+O_{2}$............... 24

$O\left({ }^{\top} D\right)+N_{2} \rightarrow O\left({ }^{3} P\right)+N_{2}$............. 28

$O\left({ }^{1} \mathrm{D}\right)+\mathrm{N}_{2} \mathrm{O} \rightarrow$ products ......... 32

$\mathrm{HO}+\mathrm{HNO}_{3} \rightarrow \mathrm{H}_{2} \mathrm{O}+\mathrm{NO}_{3}$. . . . . . . . . 37

$\mathrm{HO}+\mathrm{HO}_{2} \mathrm{NO}_{2} \rightarrow$ products . . . . . . . . 40

$\mathrm{HO}_{2}+\mathrm{NO} \rightarrow \mathrm{HO}+\mathrm{NO}_{2} \cdot$. . . . . . . . . 41

$\mathrm{NO}+\mathrm{O}_{3} \rightarrow \mathrm{NO}_{2}+\mathrm{O}_{2} \cdot . . . . . . . . . .46$

$\mathrm{NO}+\mathrm{NO}_{3} \rightarrow 2 \mathrm{NO}_{2}$. . . . . . . . . . . 50

$\mathrm{NO}_{2}+\mathrm{O}_{3} \rightarrow \mathrm{NO}_{3}+\mathrm{O}_{2}$............ 53

$\mathrm{NO}+h_{\nu}$. . . . . . . . . . . . . . 57

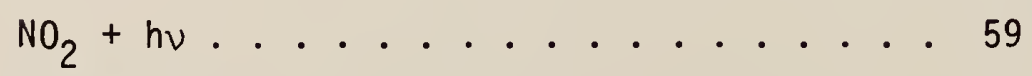

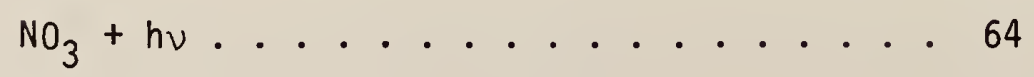

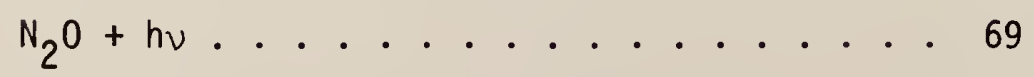

$\mathrm{N}_{2} \mathrm{O}_{5}+h \nu$. . . . . . . . . . . . 73

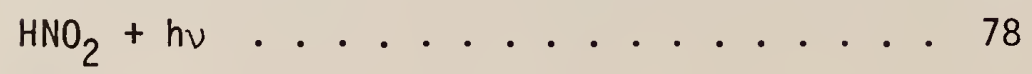

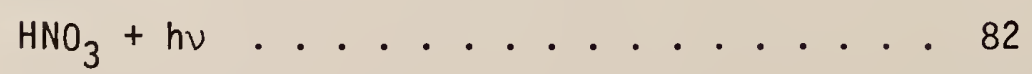

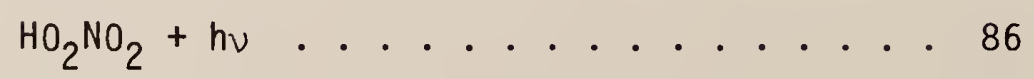




\title{
Kinetic and Photochemical Data for \\ Atmospheric Chemistry Reactions of the Nitrogen Oxides
}

\author{
R. F. Hampson
}

This report contains 24 individual data sheets for thermal and photochemical reactions of importance in the atmospheric chemistry of the nitrogen oxides. For each reaction the available experimental data are summarized and critically evaluated, and a preferred value of the rate coefficient is given. The selection of the preferred value is discussed and an estimate of its accuracy is given. For the photochemical process the data are summarized, and preferred values for the photoabsorption cross section and primary quantum yields are given. These data sheets were prepared by the author for the evaluation by the CODATA Task Group on Chemical Kinetics to be published in the Journal of Physical and Chemical Reference Data.

This work was supported in part by the High Altitude Pollution Program of the Office of Environmental Quality, Federal Aviation Adminstration and the Upper Atmospheric Physics Section of the National Aeronautics and Space Administration. It was also supported in part by the office of Standard Reference Data, NBS.

Key words: Atmospheric chemistry, chemical kinetics, data evaluation, nitrogen oxides, photoabsorption cross section, photochemistry, quantum yield, rate coefficient. 


\section{Introduction}

This report contains individual data sheets for 16 chemical reactions and 8 photochemical processes of importance in the atmospheric chemistry of the nitrogen oxides. These data sheets were prepared by the author for the evaluation by the CODATA Task Group on Chemical Kinetics to be published in the Journal of Physical and Chemical Reference Data. The Task Group convened to prepare this evaluation in a series of working sessions, the last held in May 1979 to put the manuscript in final form prior to submission for publication. The last meeting at which new data was considered for evaluation was held in December 1978. At that time the Task Group restricted its consideration to data published in the scientific literature, preprints of journal articles and significant new results presented at open scientific meetings. The evaluations contained in this report reflect the combined judgment of the entire Task Group whose membership consists of: J. A. Kerr (Chairman), D. L. Baulch, R. A. Cox, R. F. Hampson, J. Troe and R. T. Watson.

\section{Guide to the Data Sheets}

The data sheets are of two types, (i) those for the thermal reactions and $(i i)$ those for the photochemical reactions.

\subsection{Thermal Reactions}

The data sheets begin with a statement of the reactions including all pathways which are considered feasible. This is followed by the corresponding enthalpy changes at $298 \mathrm{~K}$.

The available kinetic data on the reactions are summarized under three headings, (i) Absolute Rate Coefficients, (ii) Relative Rate Coefficients 
and ( $i j i)$ Reviews and Evaluations. Some of the earlier data summarized in previous evaluations have been omitted, except where the data have a direct bearing on the preferred data selected here. Under all three of the headings above the data are presented as absolute rate coefficients. If the temperature coefficient has been measured the results are given in a temperature-dependent form over a stated range of temperatures. For bimolecular reactions the temperature dependence is expressed in the normal Arrhenius form, $k=A \exp (-C / T)$ where $C=E / R$.

Single temperature data are presented as such and wherever possible the rate coefficient at $298 \mathrm{~K}$ is quoted directly as measured by the original authors. This means that the listed rate coefficient at $298 \mathrm{~K}$ may differ slightly from that calculated from the Arrhenius parameters determined by the same authors. Rate coefficients at $298 \mathrm{~K}$ marked with an asterisk indicate that the value was calculated by extrapolation of a measured temperature range which did not include $298 \mathrm{~K}$. The data listed under Reviews and Evaluations are from the most recent source if that source contains the same recommendations as earlier reviews.

The tables of data are supplemented by a series of comments summarizing the experimental details. For measurements of relative rate coefficients the comments contain the actual measured ratio of rate coefficients together with the rate coefficient of the reference reaction used to calculate the absolute rate coefficient listed in the data table. The absolute value of the rate coefficient given in the table may be different from that reported by the original author owing to a different choice of rate coefficient of the reference reaction. Whenever possible the reference rate data is that preferred in the present evaluation.

The preferred rate coefficients are presented, (i) at a temperature of $298 \mathrm{~K}$ and $(\mathrm{i} i$ ) in temperature-dependent form over a stated range of 
temperatures. This is followed by a statement of the error limits in $\log k$ at $298 \mathrm{~K}$ and in $(E / R)$ for the temperature range of the preferred rate coefficient. Some comments on the assignment of errors are given later in this Introduction.

The "Comments" on the preferred values describe how the selection was made and give any other relevant information. The extent of the comments depends upon the present state of our knowledge of the particular reaction in question. The data sheets are concluded with a list of the relevant references.

\subsection{Photochemical Reactions}

The data sheets begin with a list of feasible primary photochemical transitions for wavelengths usually down to $170 \mathrm{~nm}$, along with the corresponding enthalpy changes at $0 \mathrm{~K}$ and calculated threshold wavelengths. This is followed by tables summarizing the avilable experimental data on, ( $i$ ) absorption cross sections and (ii) quantum yields. These data are supplemented by a series of comments.

The next table lists the preferred absorption cross section data and the preferred quantum yields at wavelength intervals of $5 \mathrm{~nm}$ where possible. The comments again describe how the preferred data were selected and include any other relevant points. The photochemical data sheets are also concluded with a list of references.

\subsection{Asignment of Errors}

Under the heading "reliability", estimates have been made of the absolute accuracies of the preferred values of $k$ at $298 k$ and of the preferred values of $E / R$ over the quoted temperature range. The accuracy of the 
preferred rate coefficient at $298 \mathrm{~K}$ is quoted as the term $\Delta \log \mathrm{k}$, where $\Delta \log$ $k=D$ and $D$ is defined by the equation, $\log _{10} k=C \pm D$. This is equivalent to the statement that $k$ is uncertain to a factor of $F$ where $D=\log _{10} F$. The accuracy of the preferred value of $E / R$ is quoted as the term $\Delta(E / R)$, where $\Delta(E / R)=G$ and $G$ is defined by the equation $E / R=H \pm G$.

The assignment of these absolute error limits in $k$ and $E / R$ is a subjective assessment of the evaluators. Experience shows that for rate measurements of atomic and free radical reactions in the gas phase, the precision of the measurements, i.e. the reproducibility, is usually good. Thus, for single studies of a particular reaction involving one technique, standard deviations, or even $90 \%$ confidence limits, of $\pm 10 \%$ or less are frequently reported in the literature. Unfortunately, when evaluators come to compare data for the same reaction studied by more than one group of investigators and involving different techniques, the rate coefficients often differ by a factor of two or even more. This can only mean that one or more of the studies has involved large systematic errors which are difficult to detect. This is hardly surprising since, unlike say molecular reactions, it is not possible to study atomic and free radical reactions in isolation and consequently mechanistic and other difficulties frequently arise.

The arbitrary assignment of errors made here is based mainly on our state of knowledge of a particular reaction which is dependent upon factors such as the number of independent investigations made and the number of different techniques used. On the whole, our assessment of error limits errs towards the cautious side. Thus, in the case where a rate coefficient has been measured by a single investigation using one particular technique and is unconfirmed by independent work, we suggest that minimum 
error limits of $\pm 100 \%$ are appropriate. At the present time we do not feel justified in assigning error limits to the parameters reported for the photochemical reactions. 


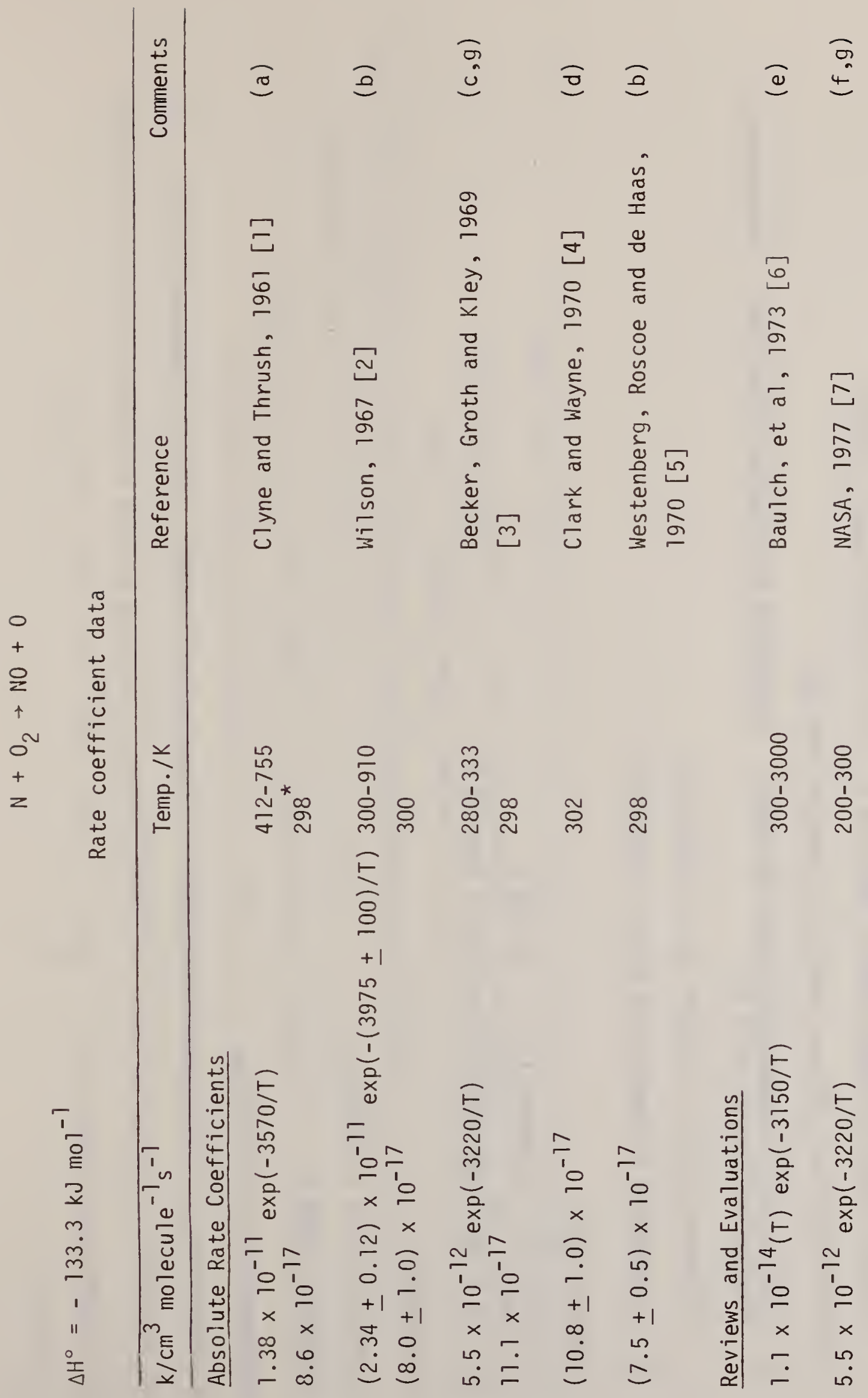




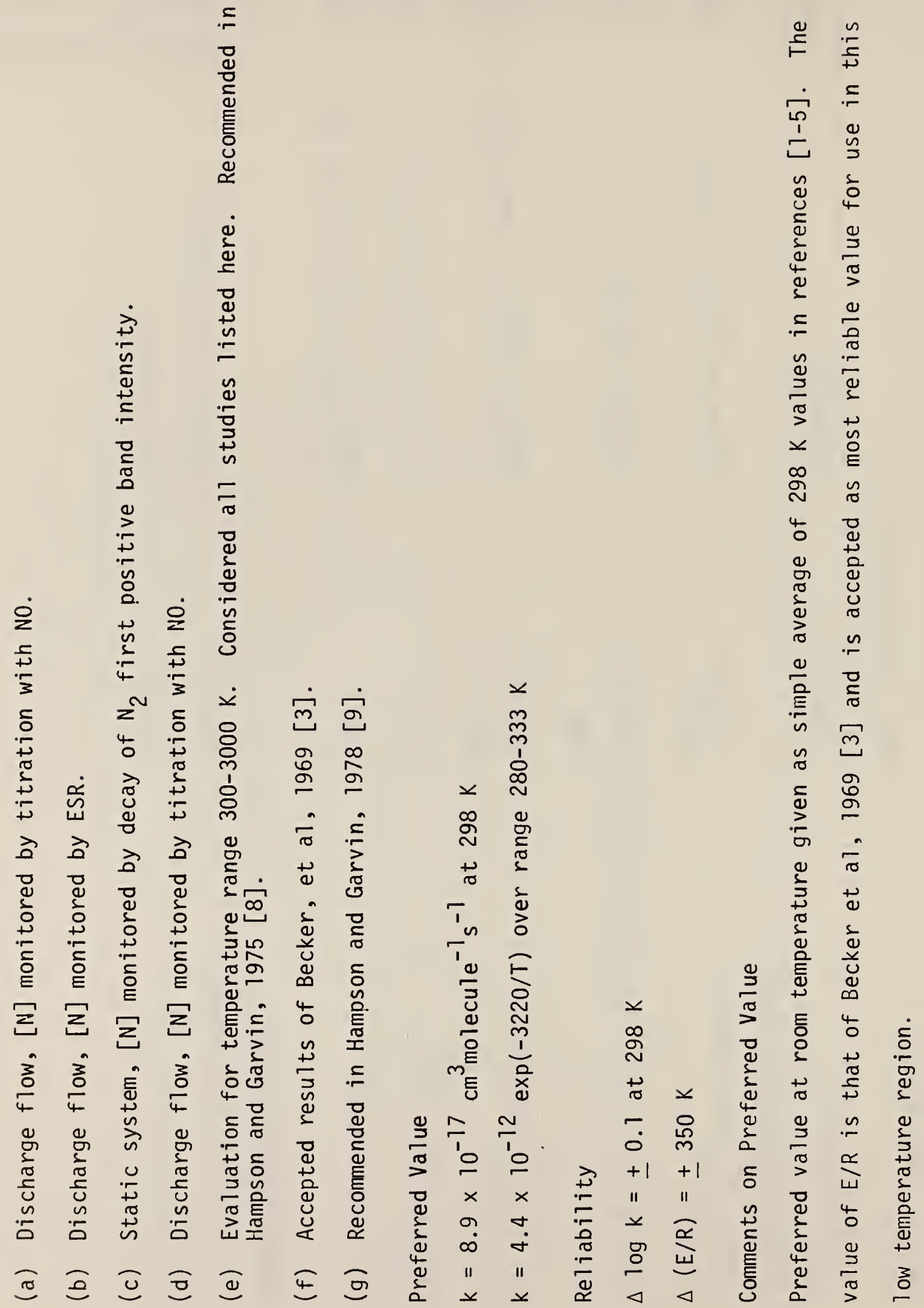




$$
\begin{aligned}
& \stackrel{\dot{\Omega}}{\stackrel{\rho}{\varrho}}
\end{aligned}
$$

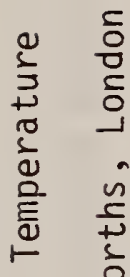

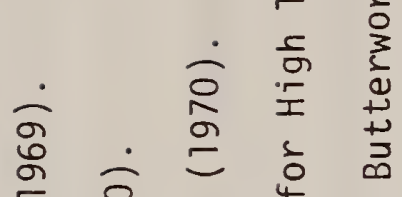

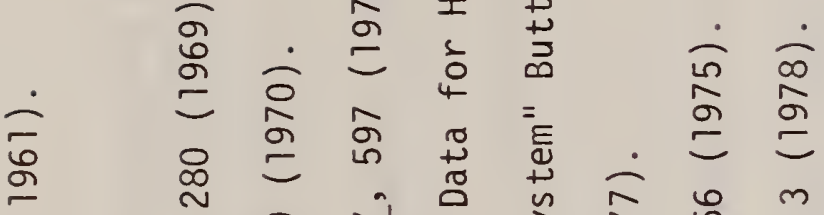

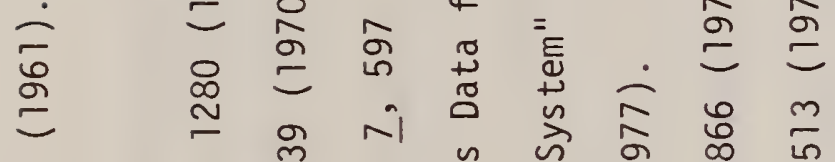

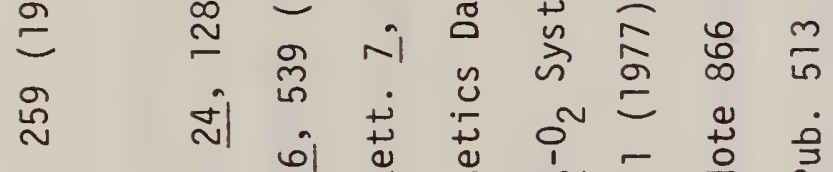

$$
\begin{aligned}
& \text { 兽 }
\end{aligned}
$$

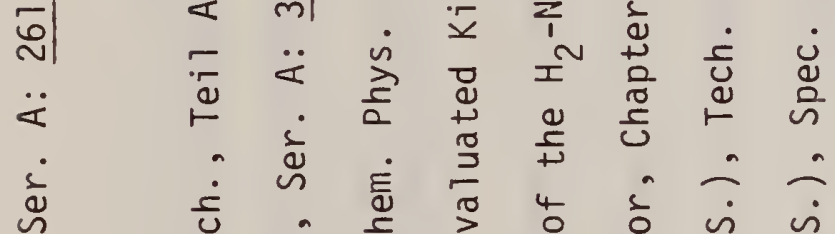

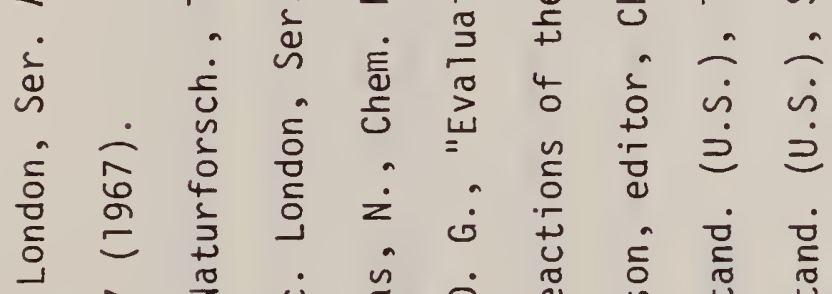

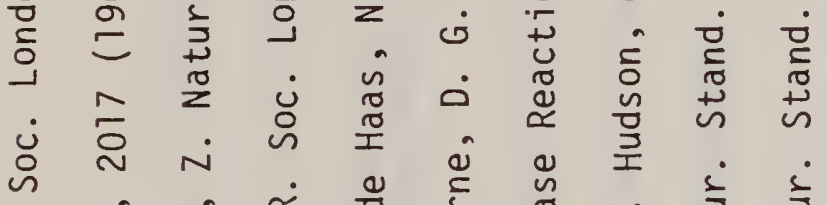

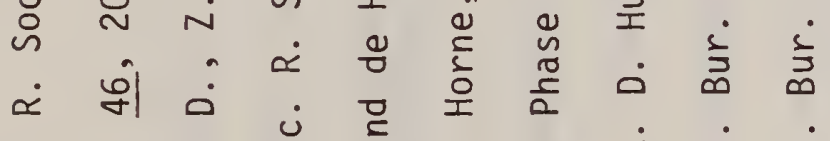

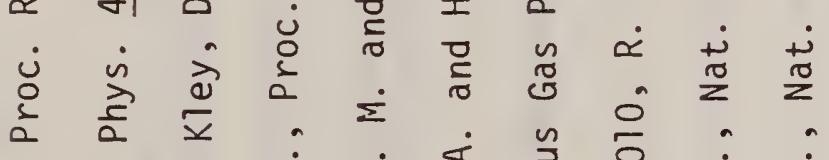

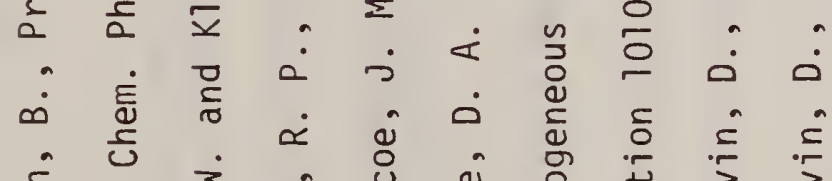

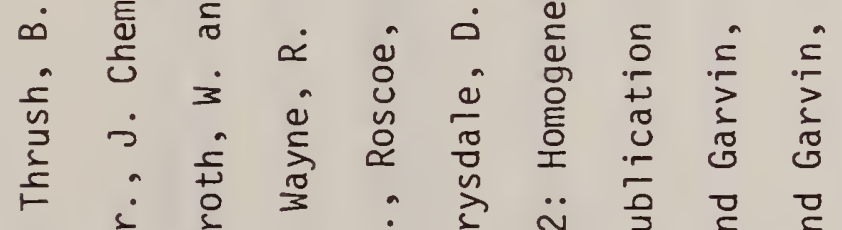

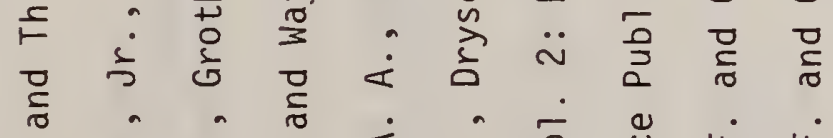

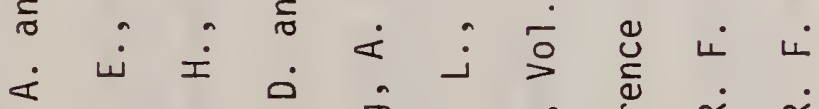

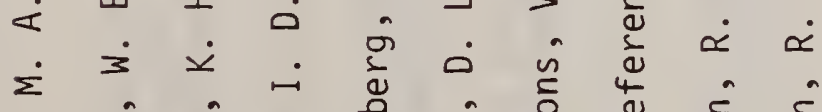

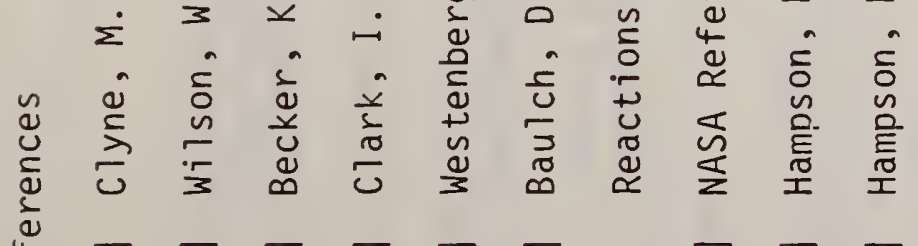

$$
\begin{aligned}
& \text { 这 } \Xi \Xi \Xi \Xi \Xi \Phi \Xi
\end{aligned}
$$




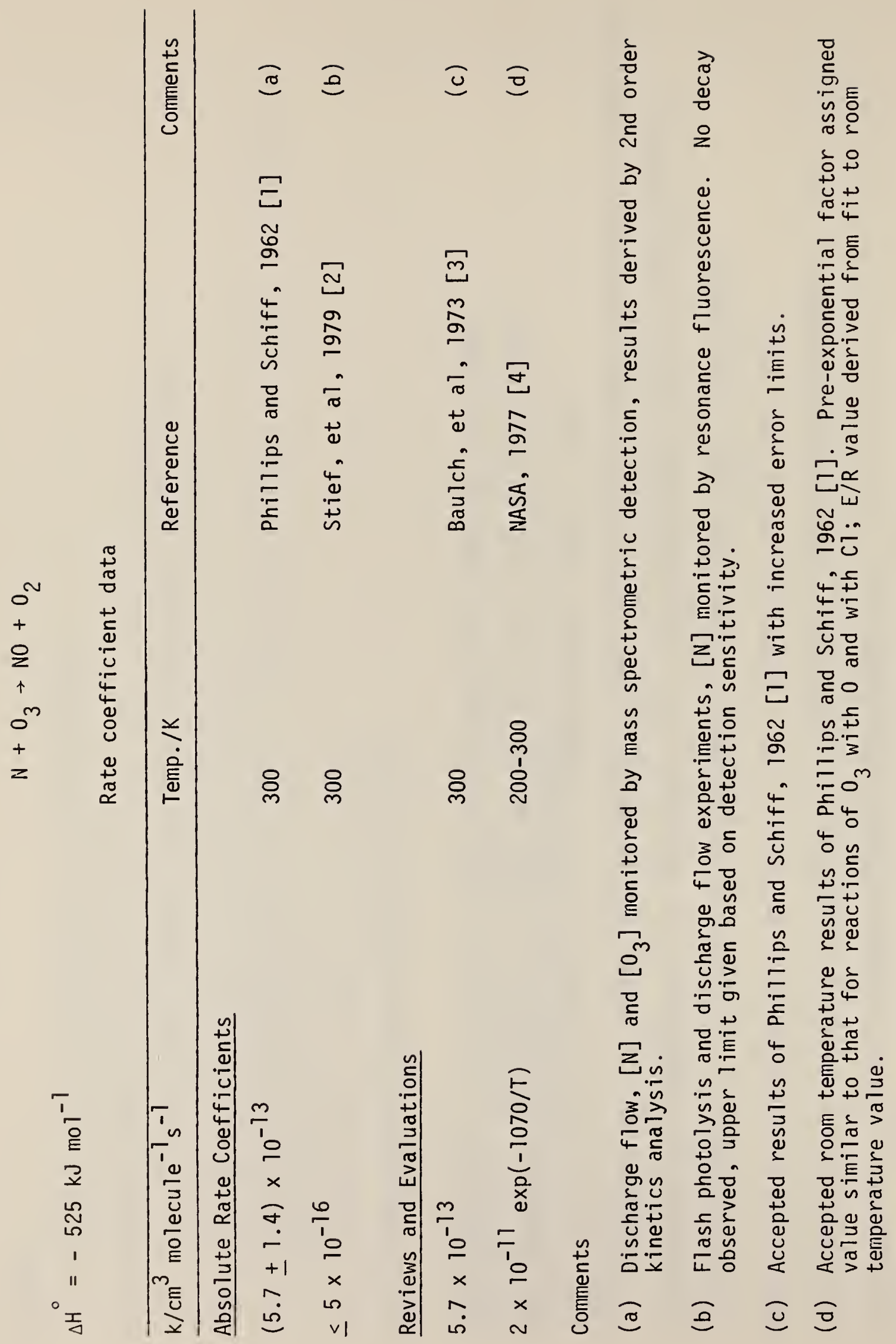




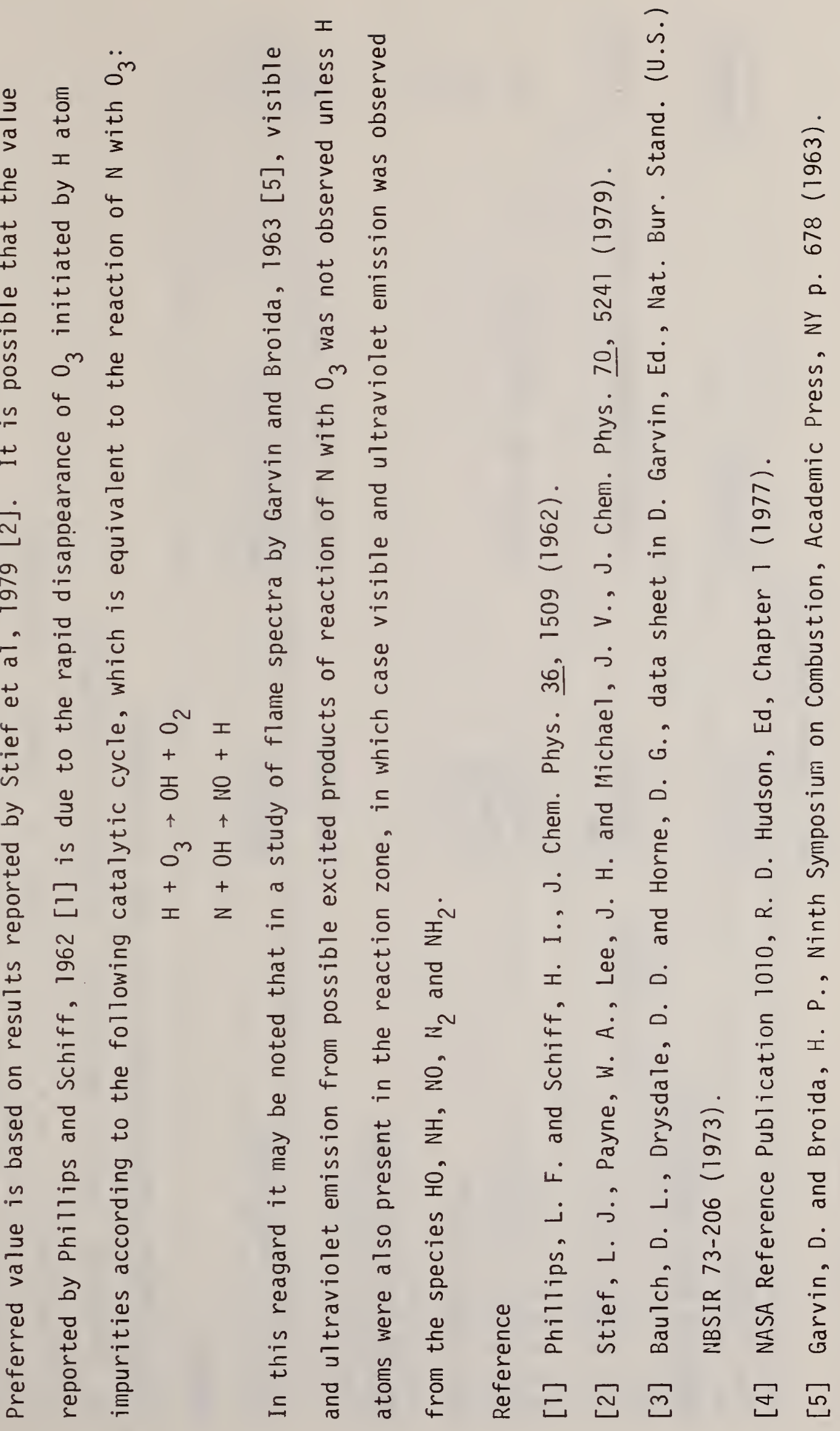




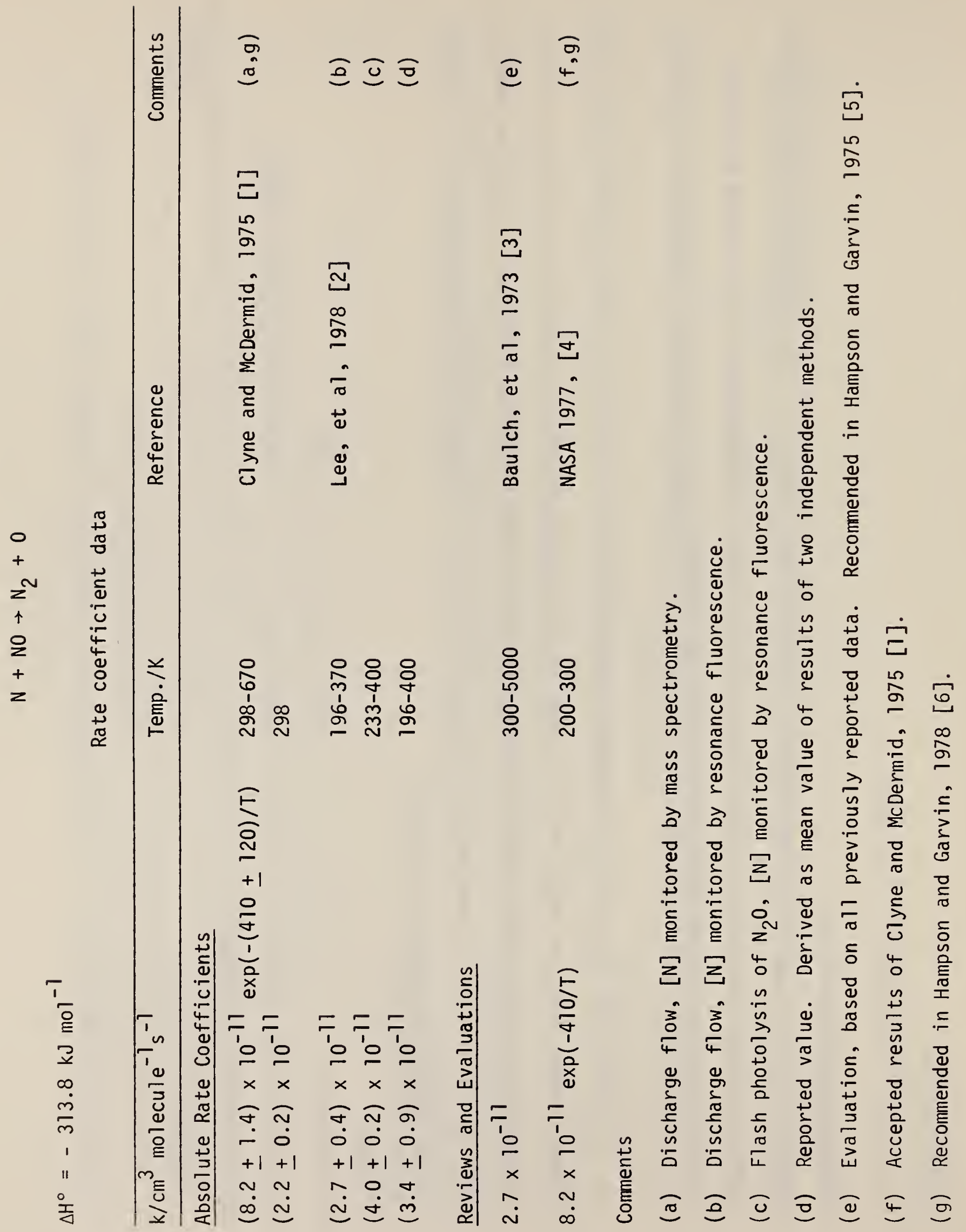




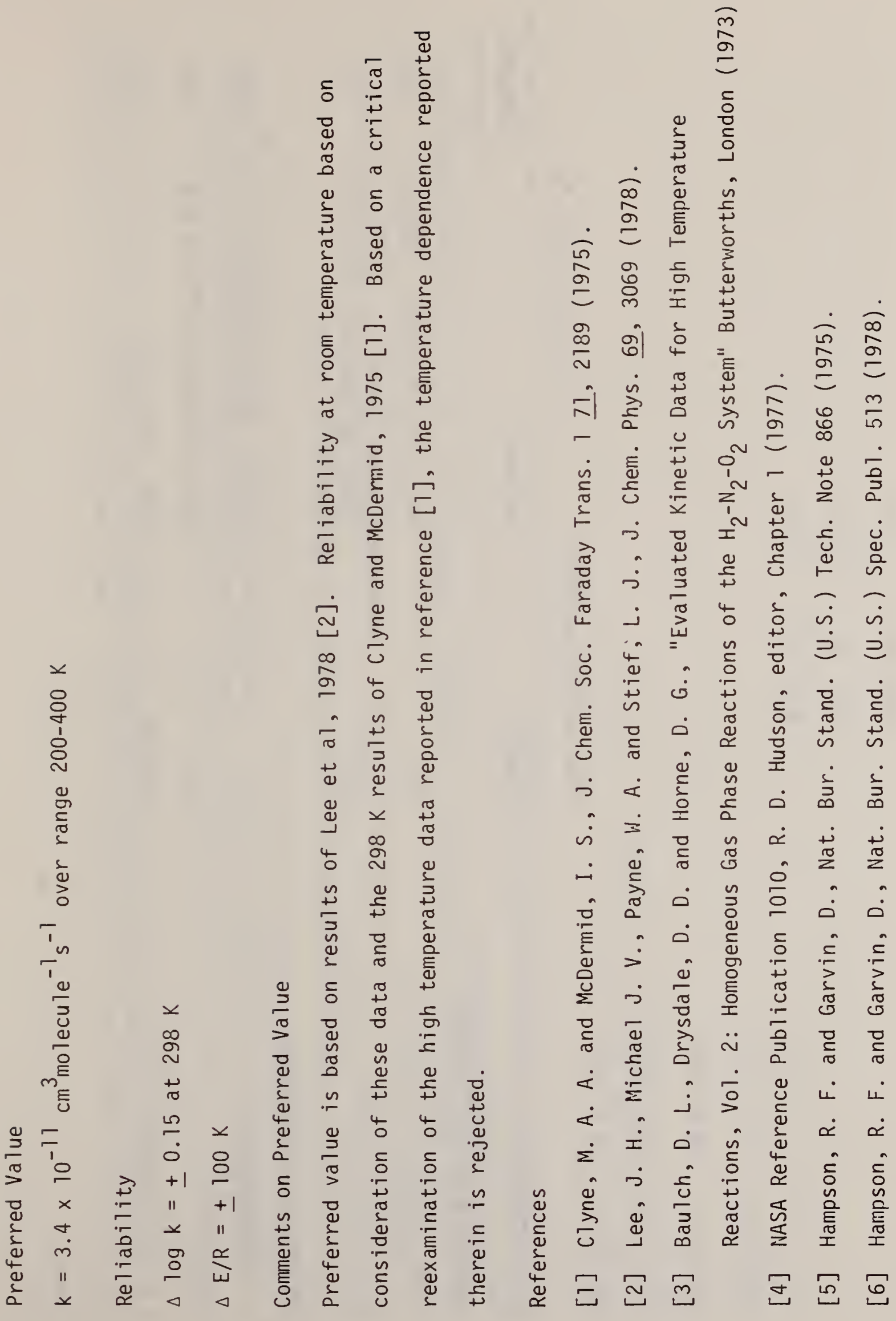




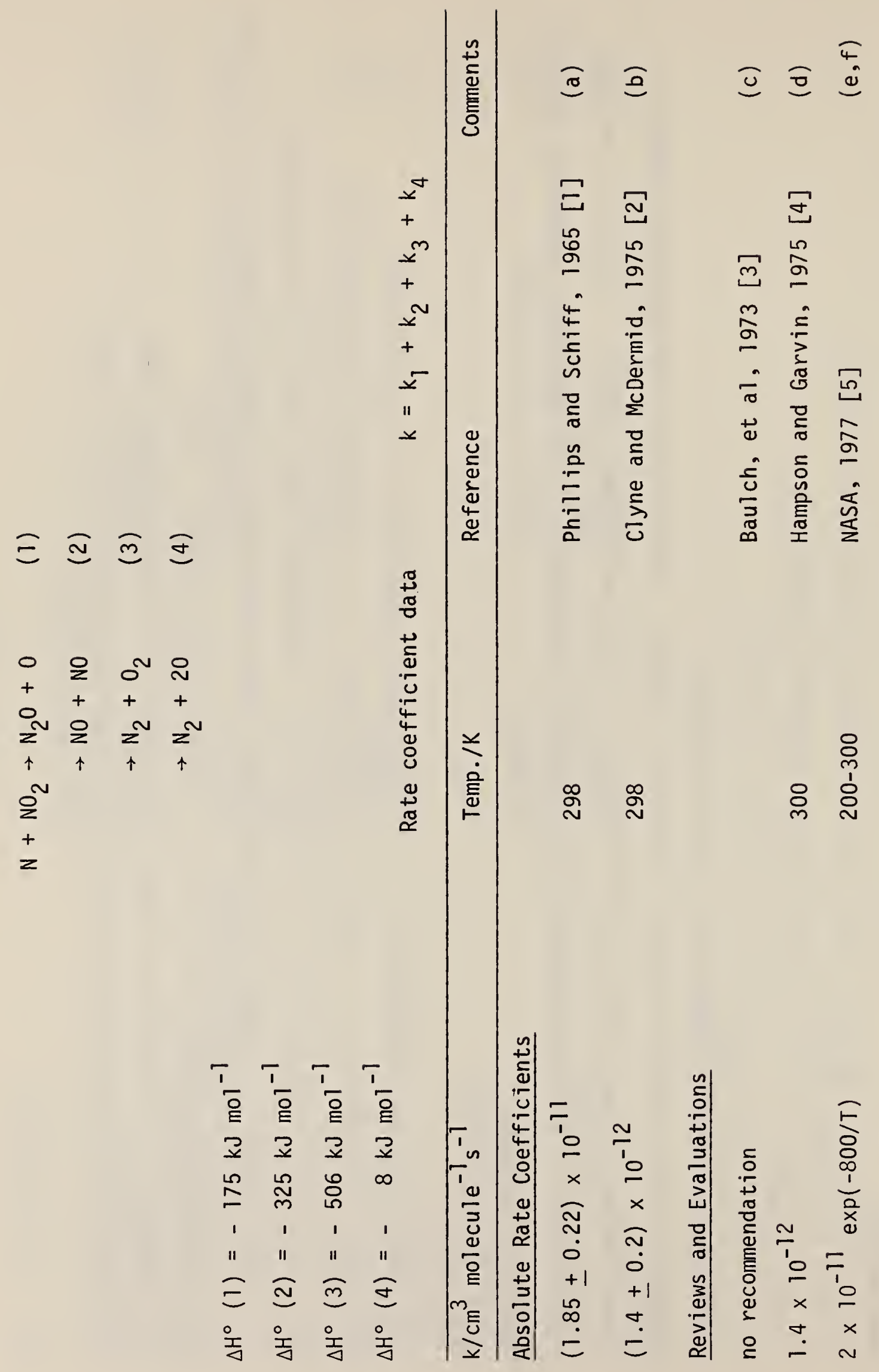


$\frac{0}{2}$

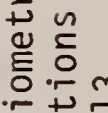

它节官

등 대

$+\frac{2}{+2}$

등 z

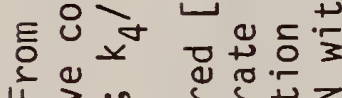

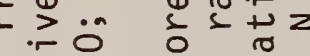

.

之 $\frac{0}{0}$ 등 릉

凹

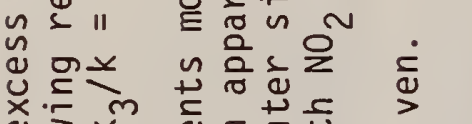

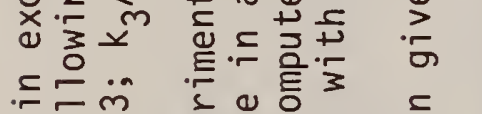

. $5 \mathrm{~m}$ \& थ

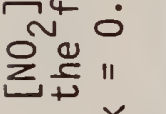

प्ष

¿ $\frac{5}{0} x$

$+\underline{w} \dot{m}$

들 등

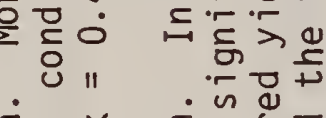

ब 仓

ه.

$\frac{\pi}{2}+40$

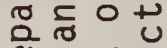

ง $\frac{1}{4} \frac{0}{8}$

है

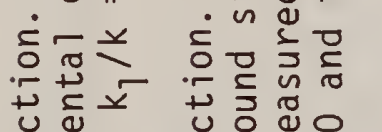

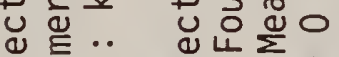

世ै

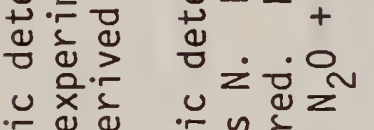

$\dot{z} \dot{0}$

당

屯ै

言 ¿े

कैष

近

जิ

$\sim \frac{1}{\Phi} \frac{c}{\sigma}$

苋放

है잉

离

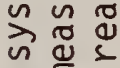

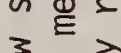

造

$\underline{U}=\frac{1}{3} 0$

जे त्राए

는 造

믈 을

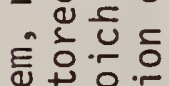

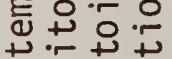

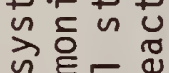

ज ह

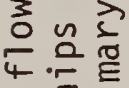

政

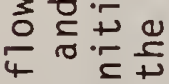

ه 들

응

유유 늘

它 n

它先岳

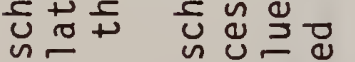

艺

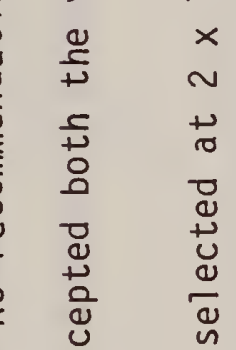

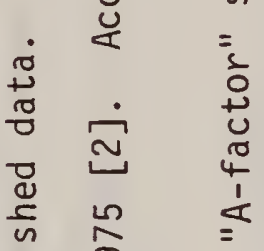

i

의

त हैं है

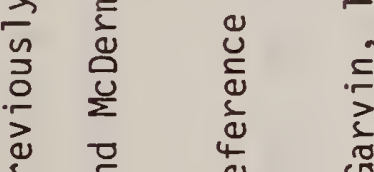

हे एँ

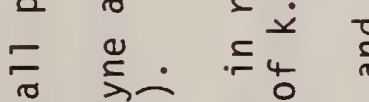

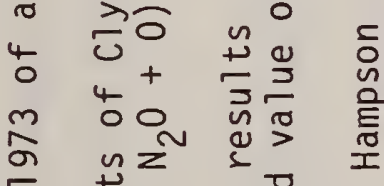

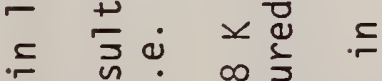

¿

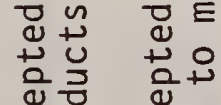

D

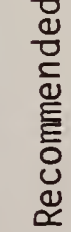

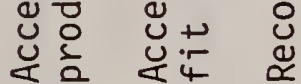

$\stackrel{0}{\frac{0}{2}} \frac{0}{1}$

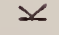

$\stackrel{\infty}{\sim}$

$+$

T'

$\frac{\frac{0}{3}}{\frac{0}{0}}$

$\stackrel{\infty}{\sim}$

마 $\times+$

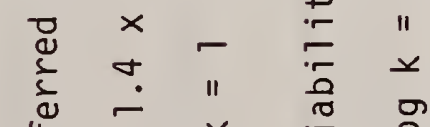

శ

อ

ฮ

a 


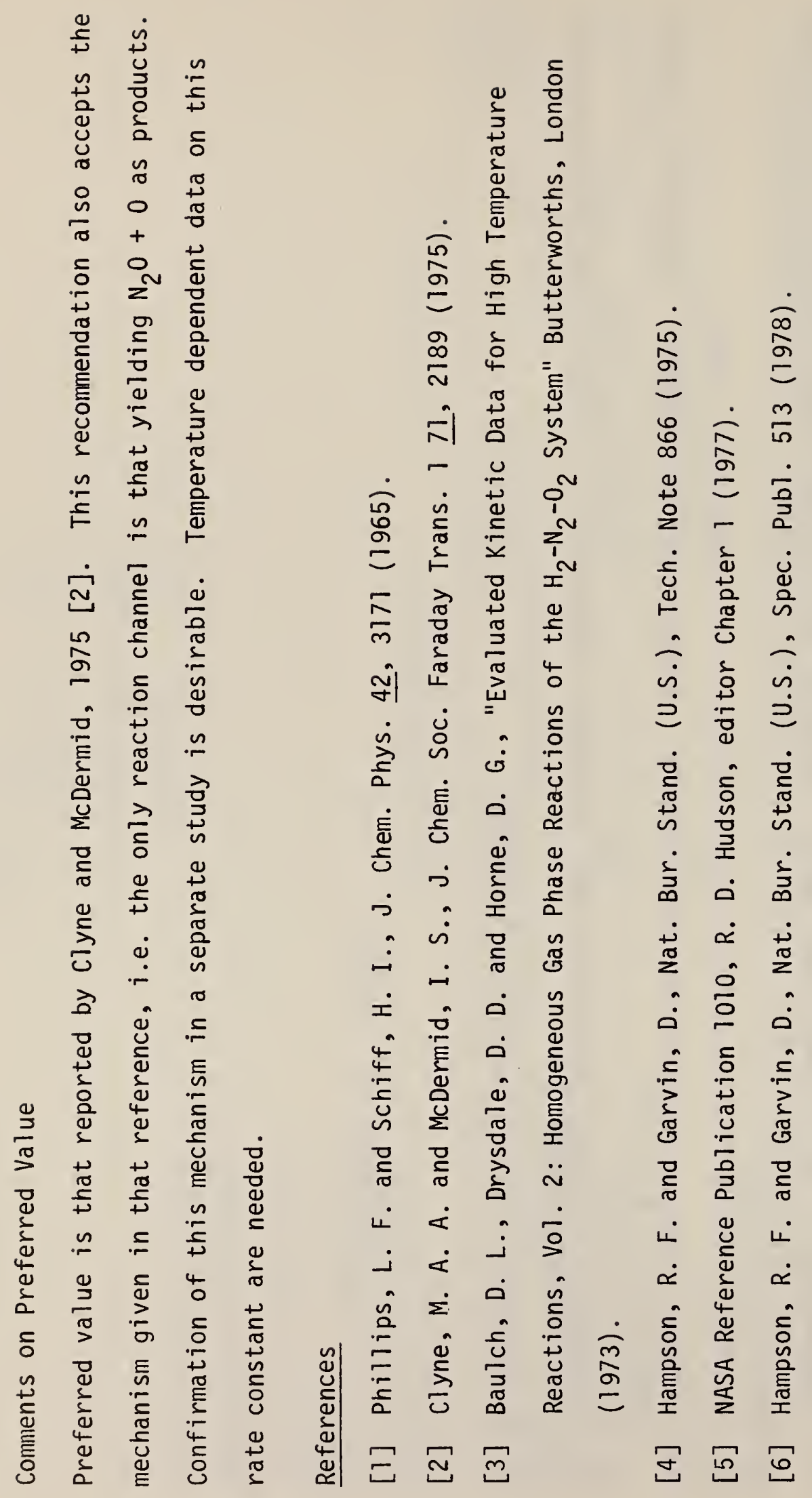




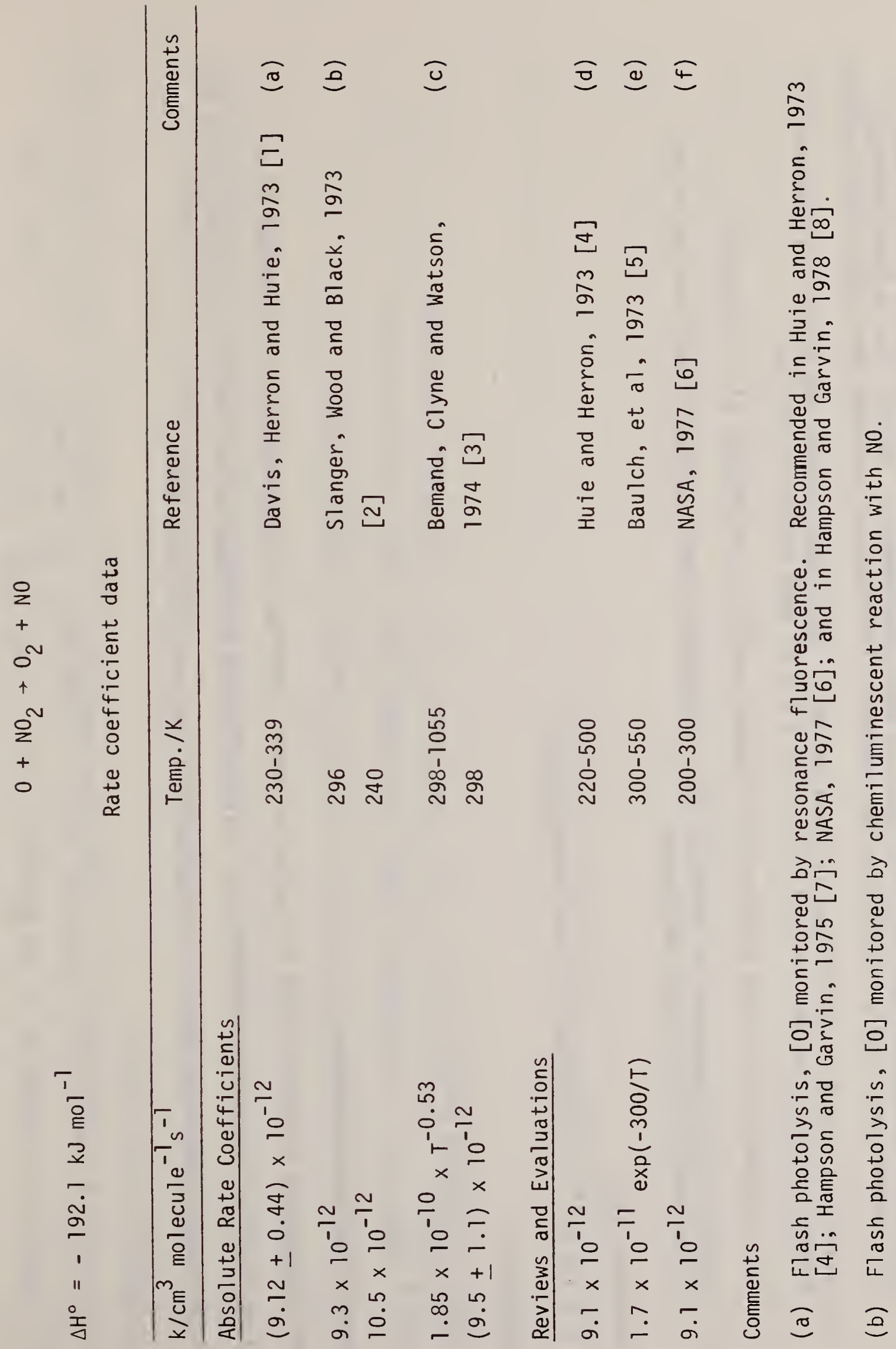




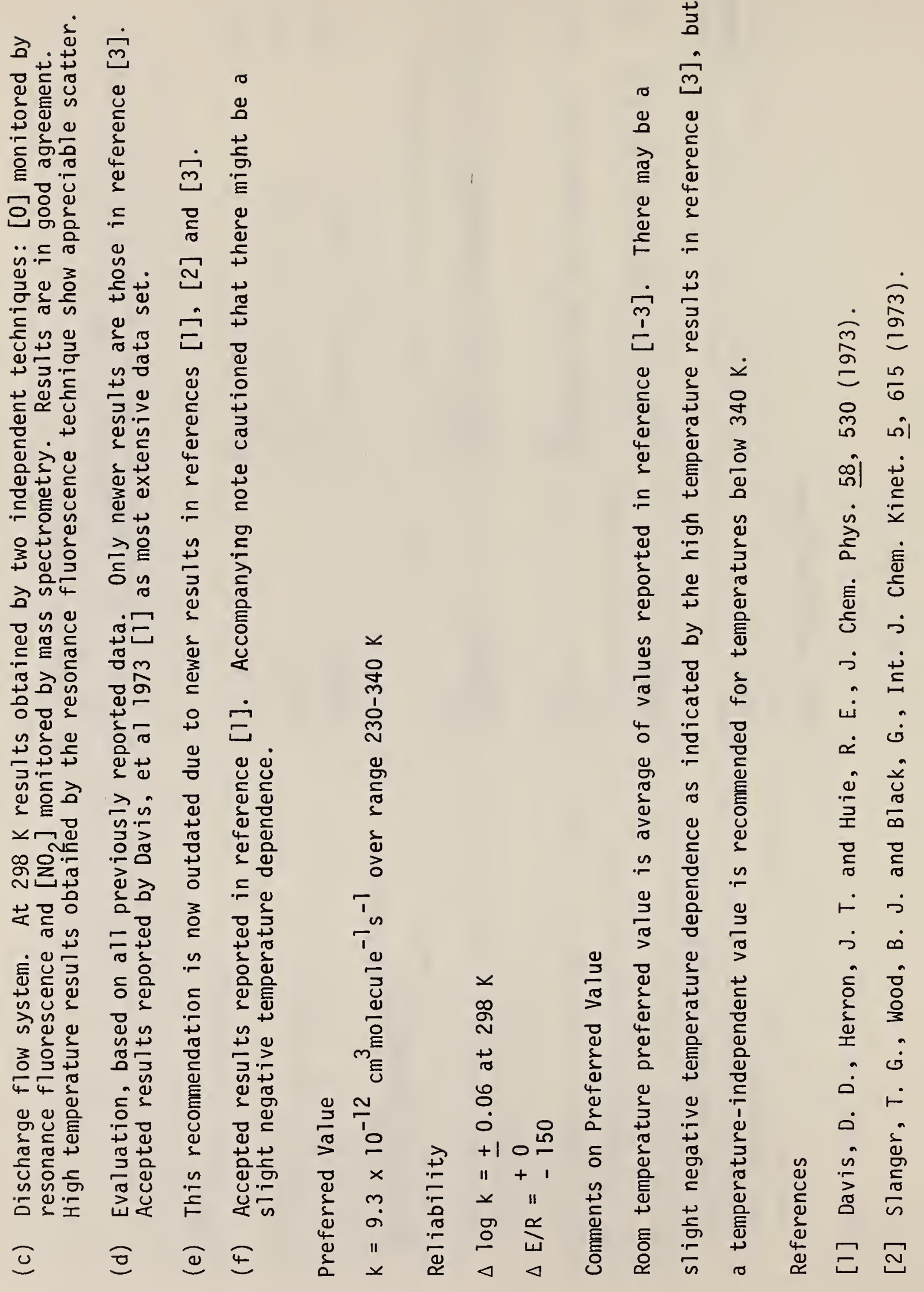




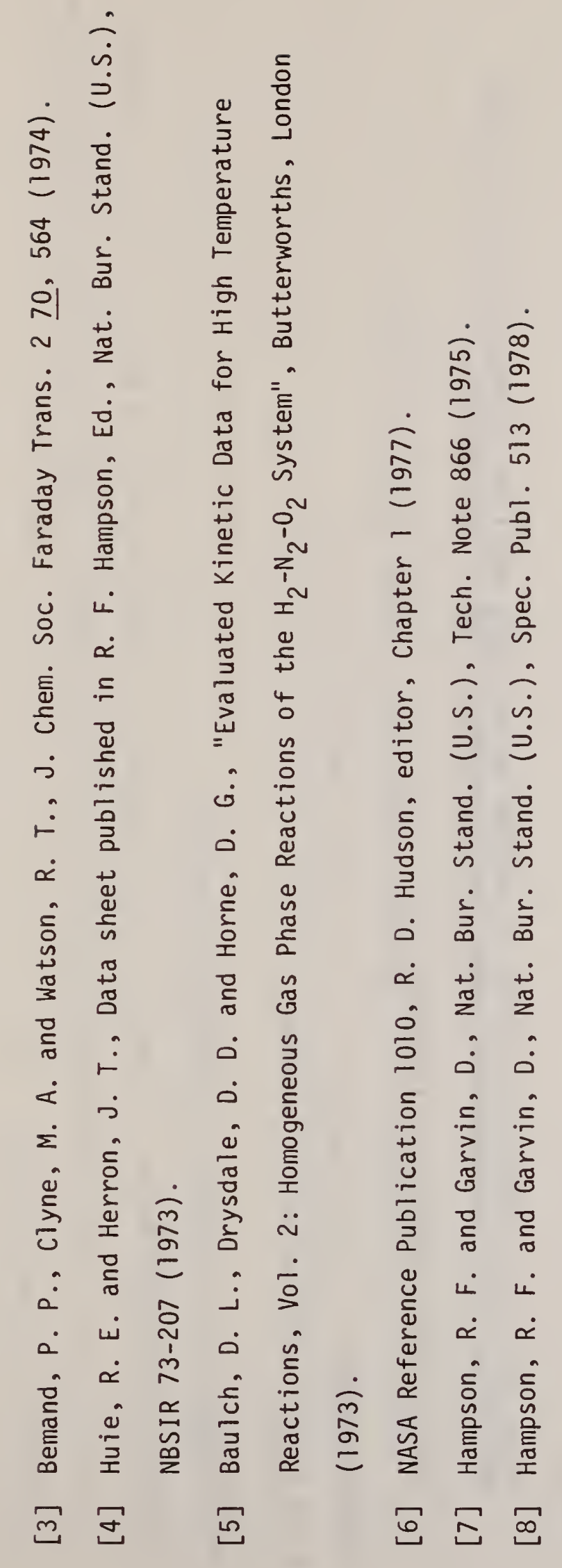




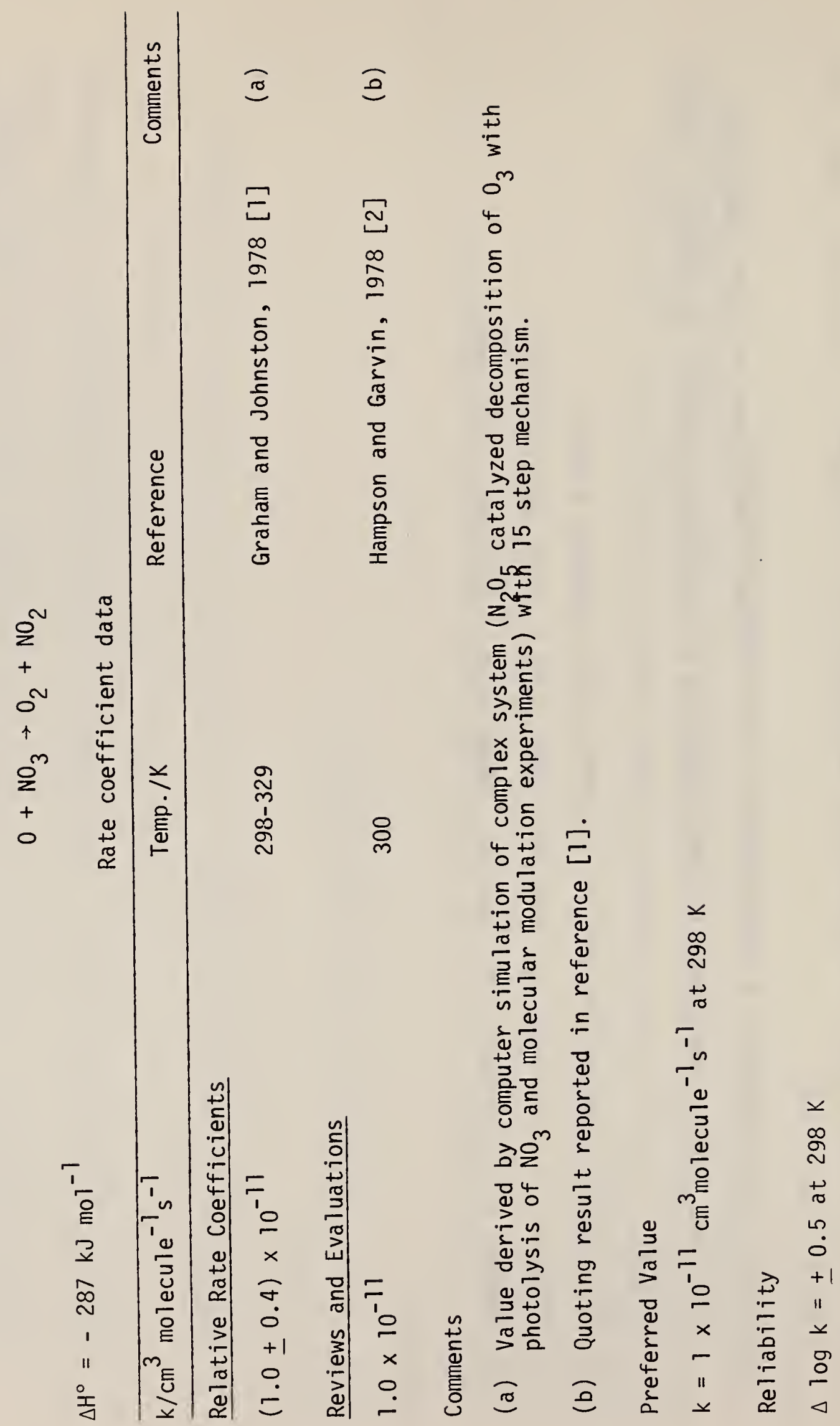




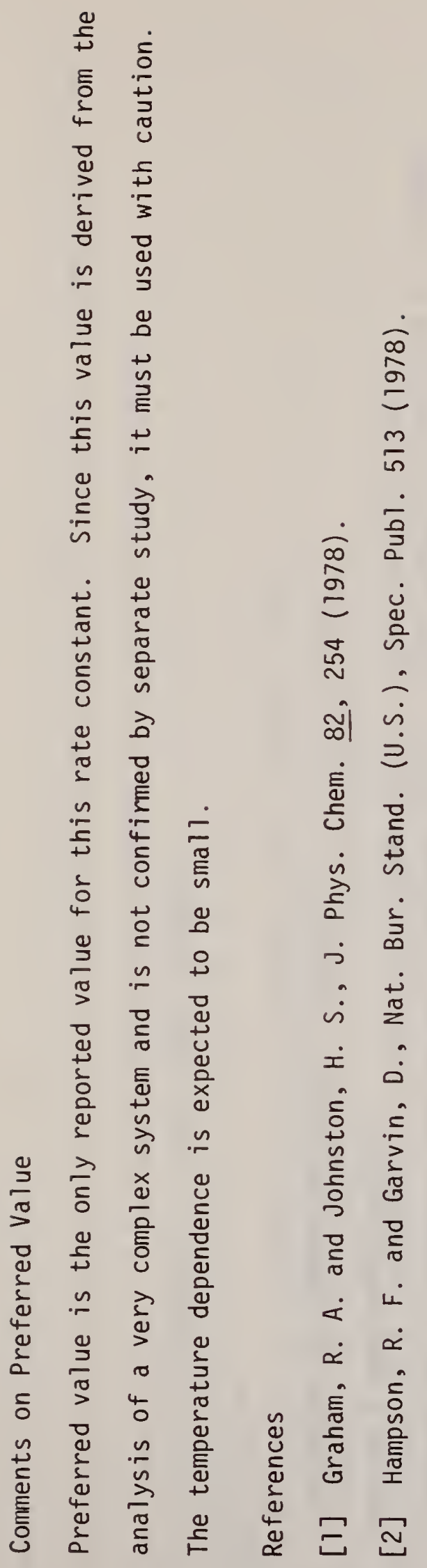




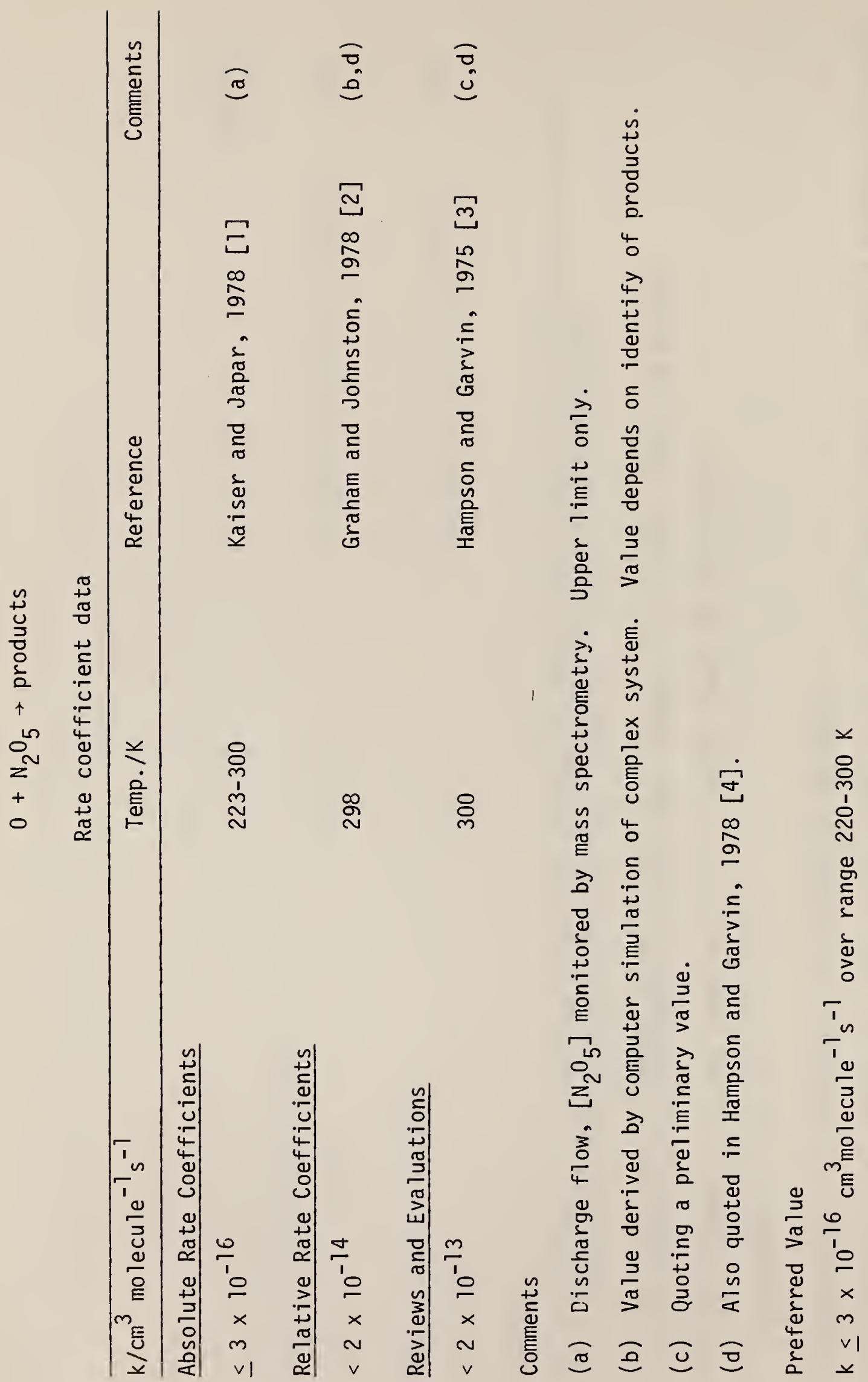




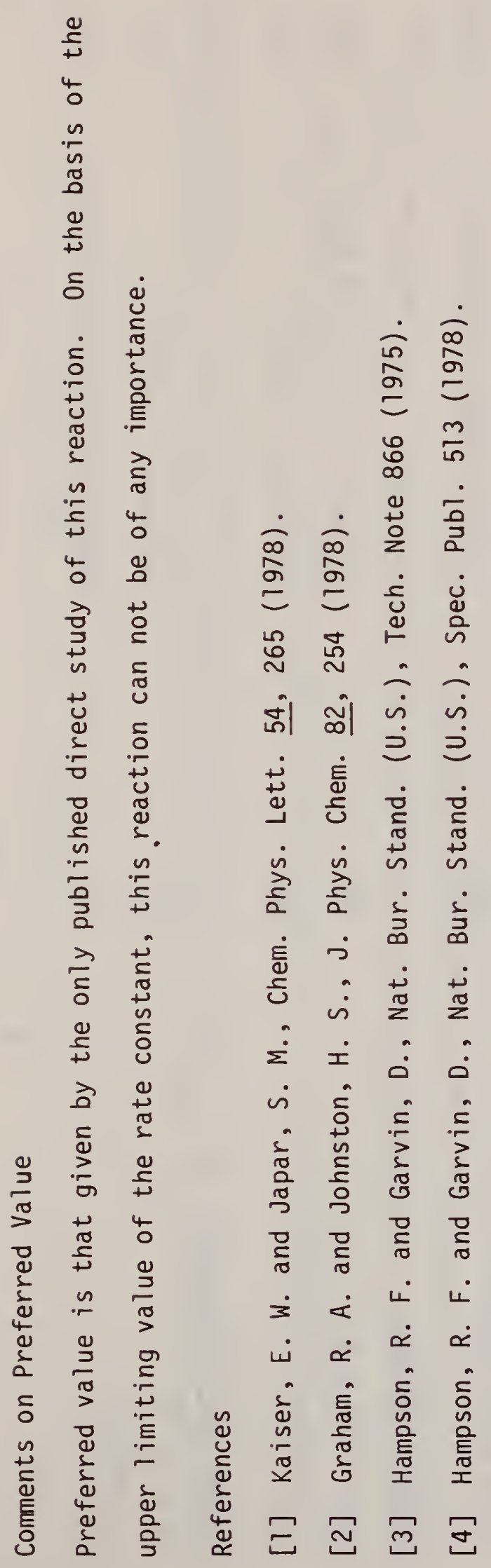




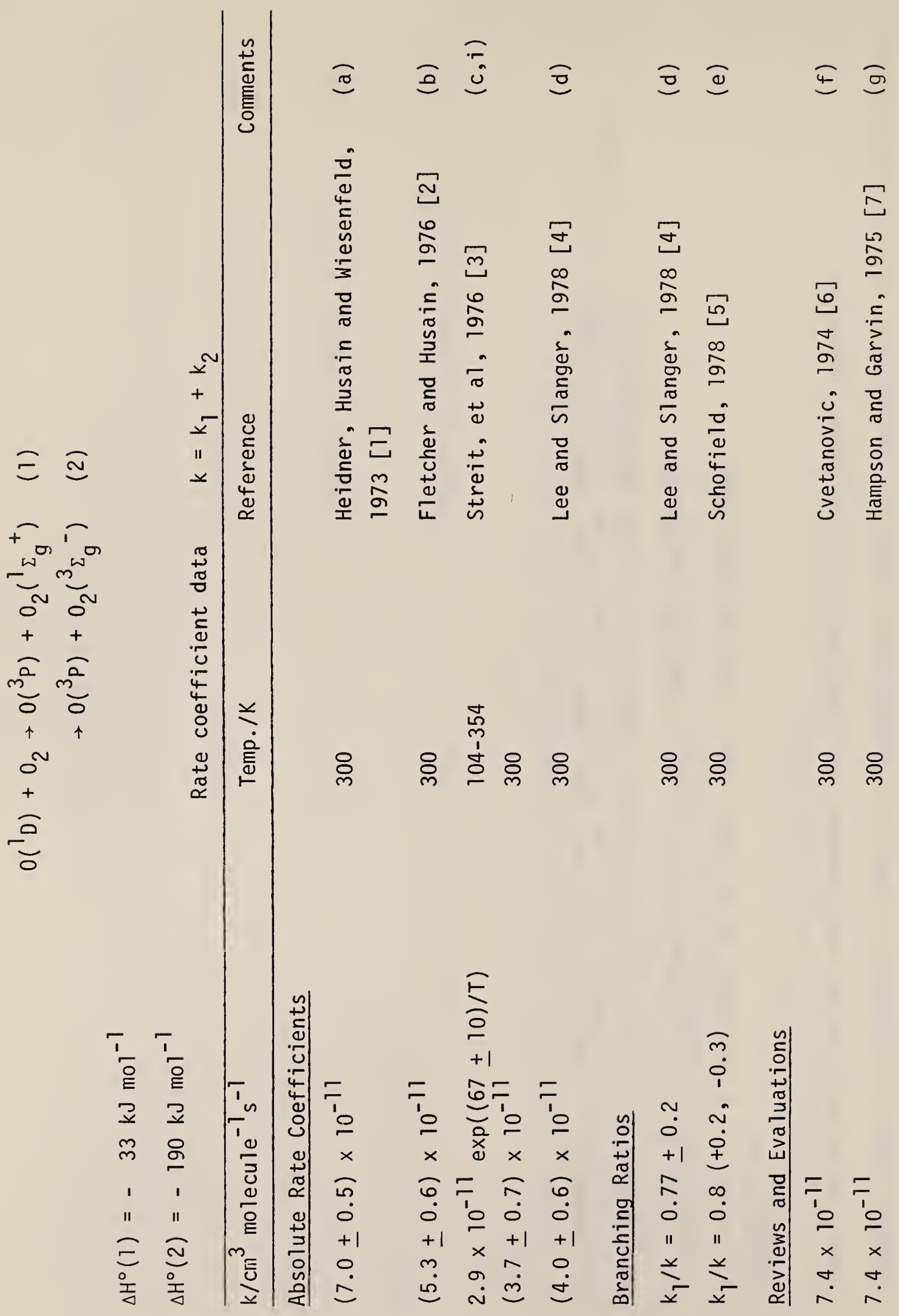




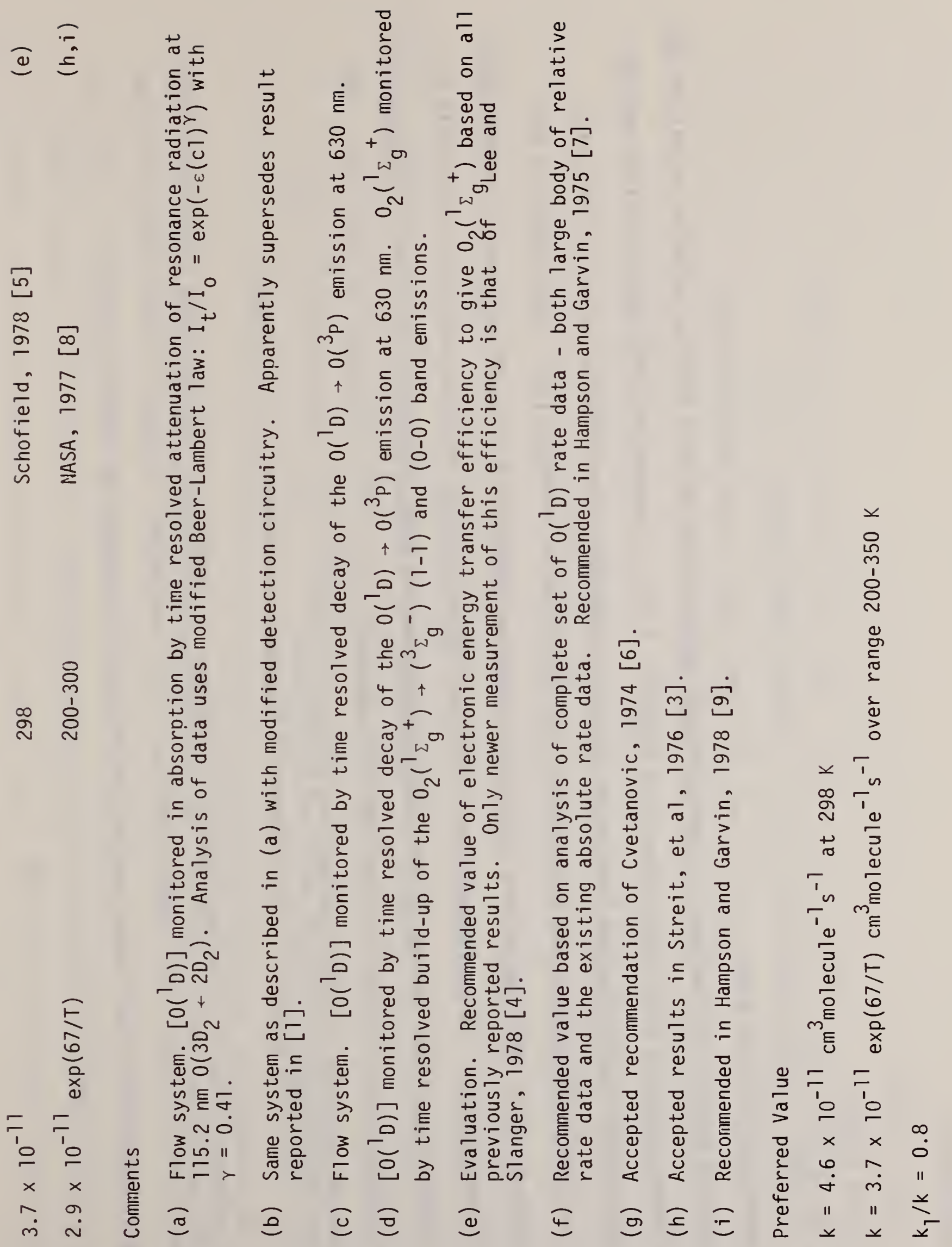




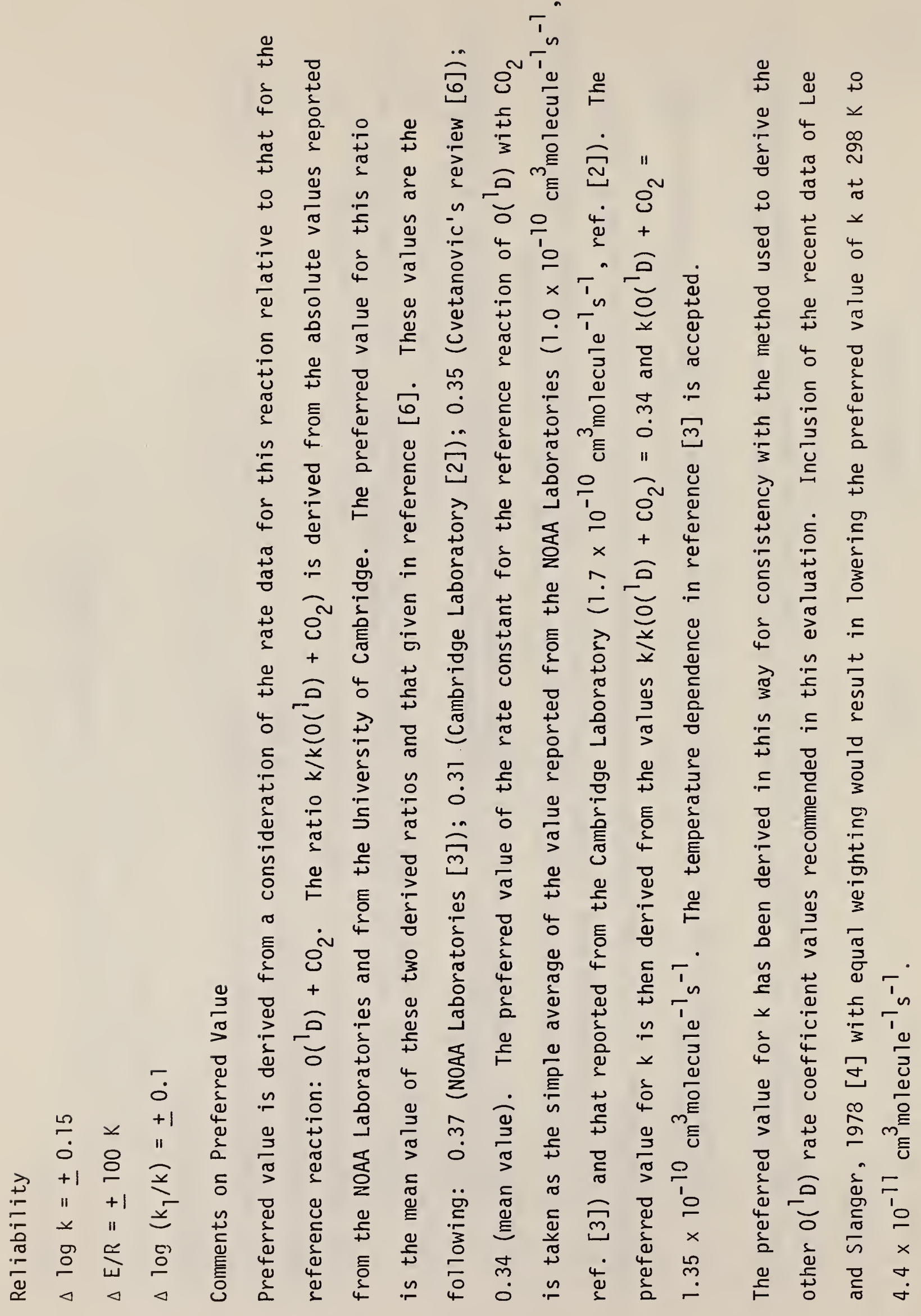




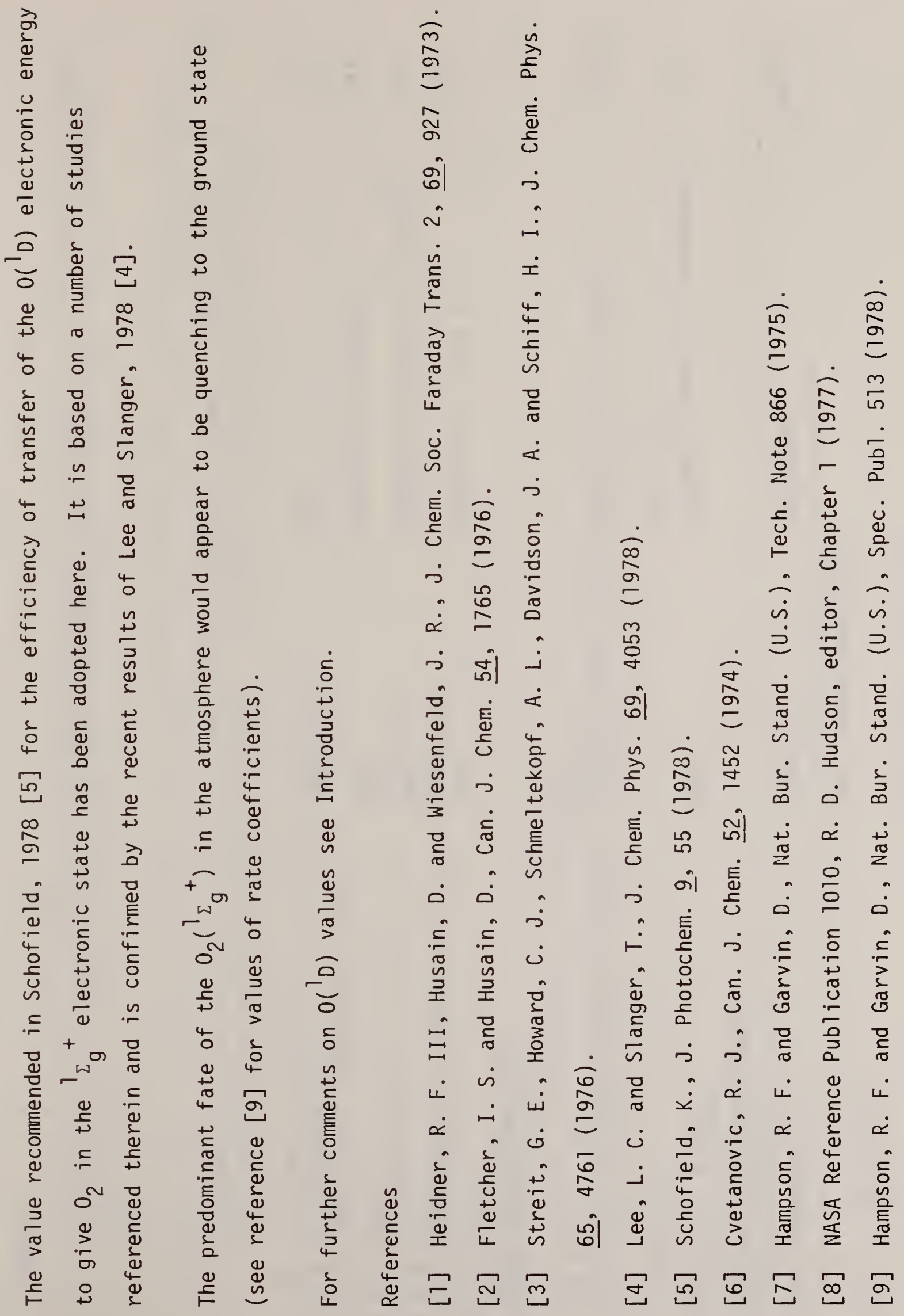




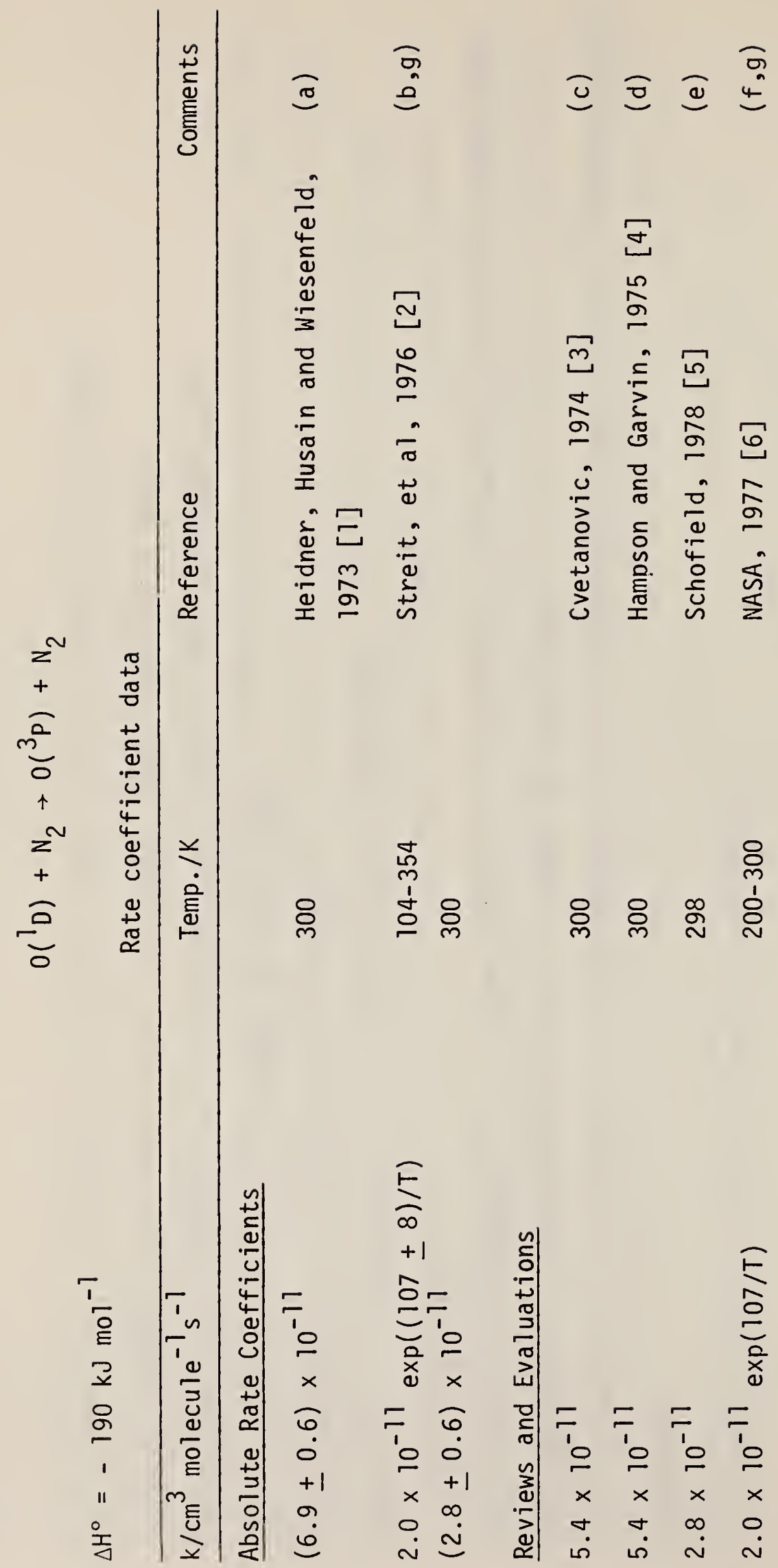




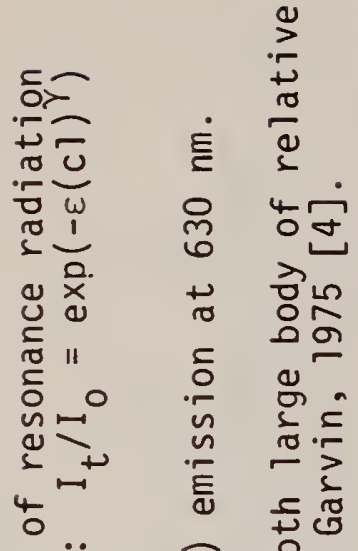

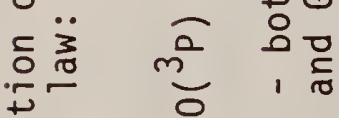

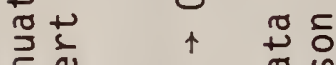

$$
\begin{aligned}
& \text { 可 } \\
& \text { 范 }
\end{aligned}
$$

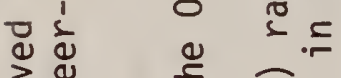

$$
\begin{aligned}
& \geqslant \infty
\end{aligned}
$$

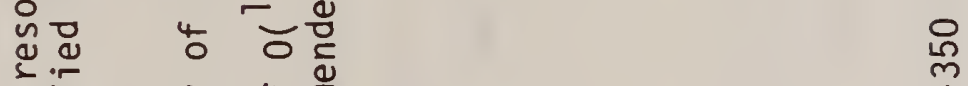

$$
\begin{aligned}
& \text { 至 }
\end{aligned}
$$

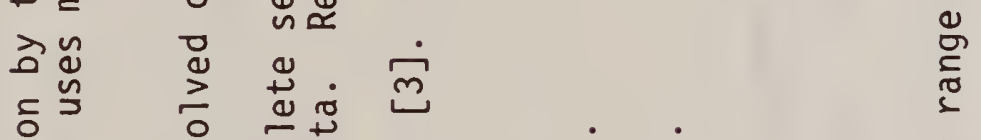

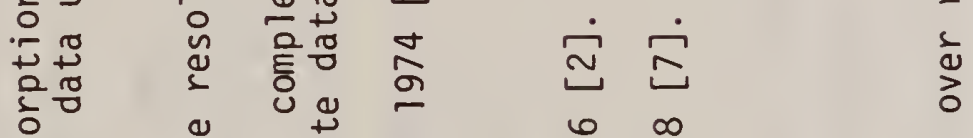

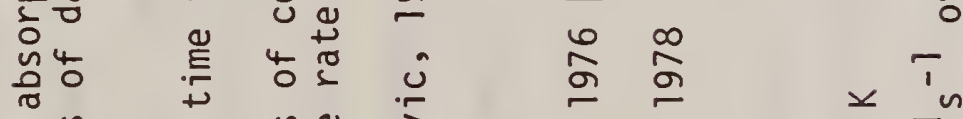

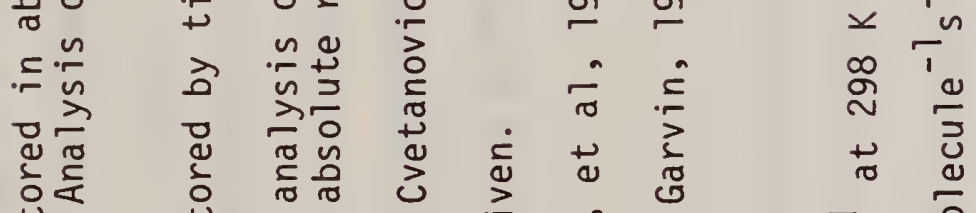

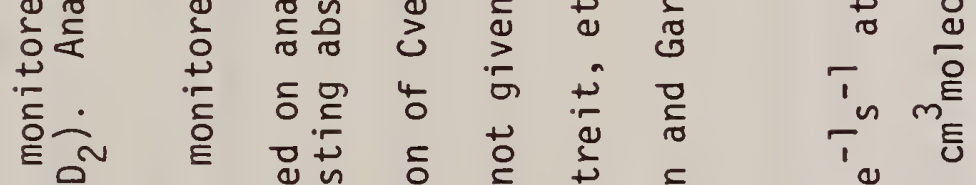

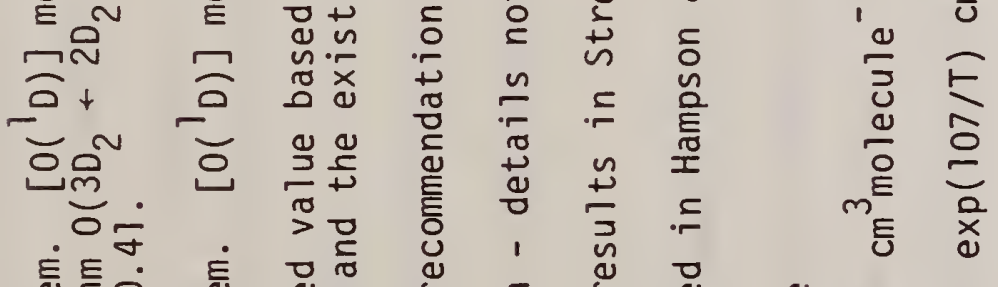

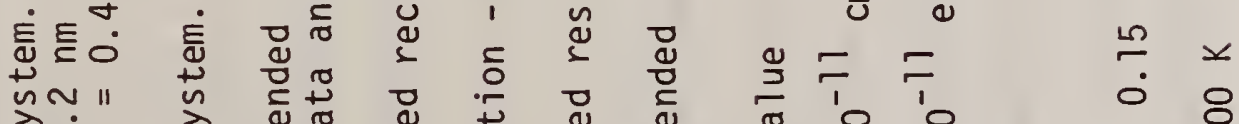

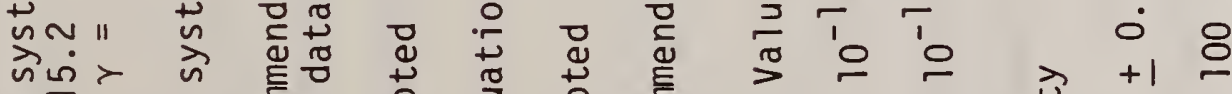

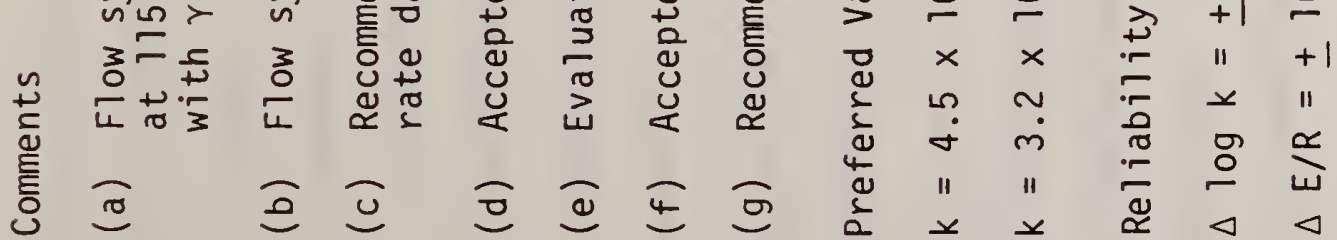




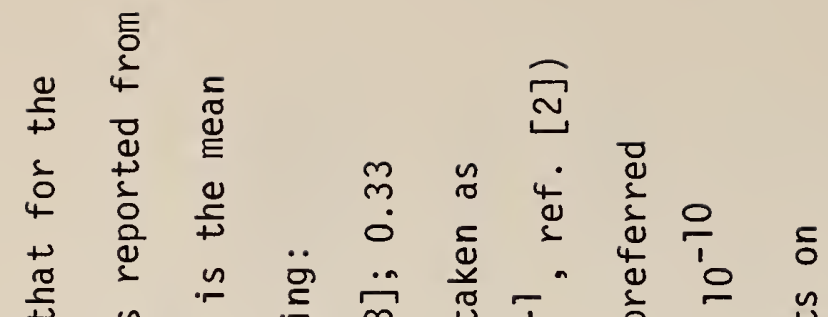

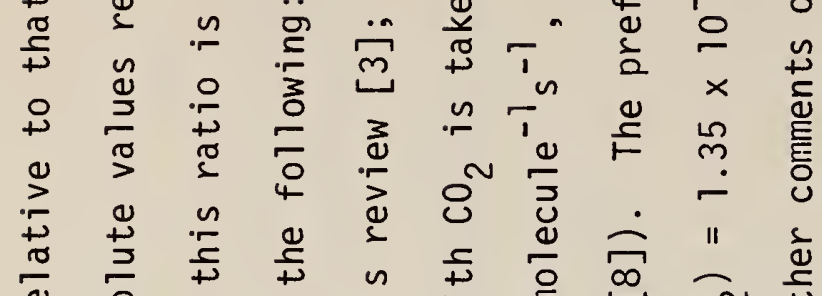

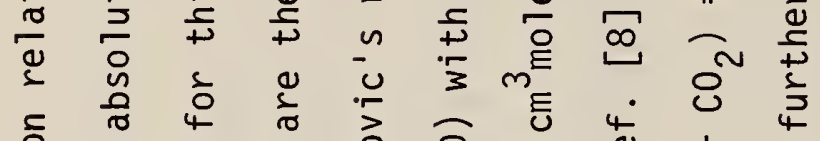
ᄃ 峁

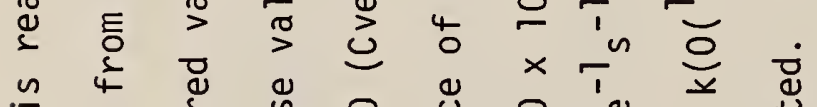

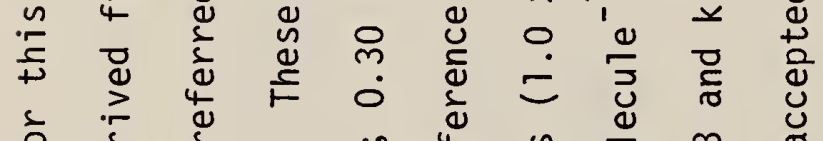

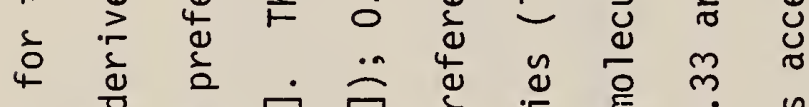

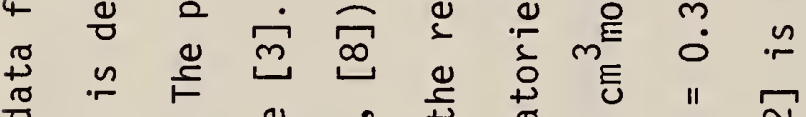

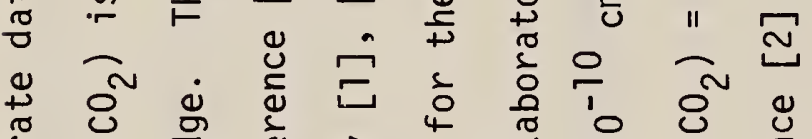

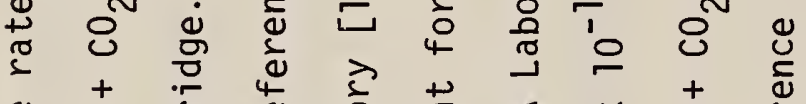

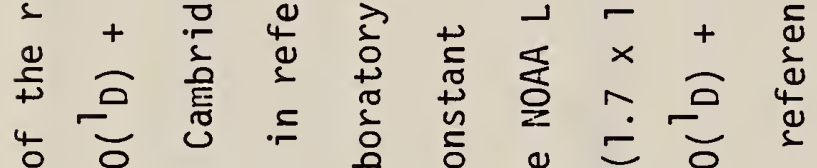

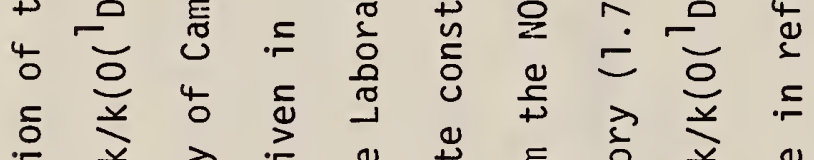

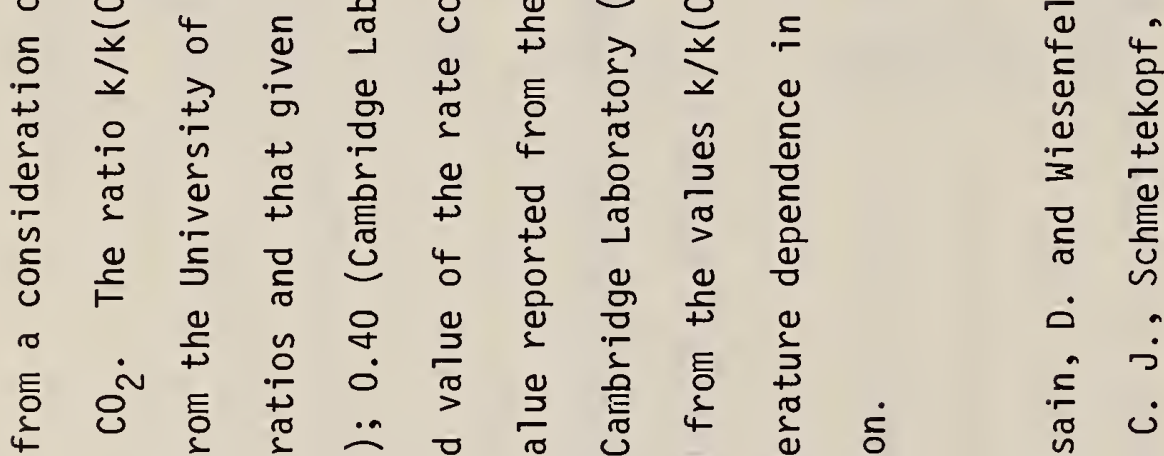

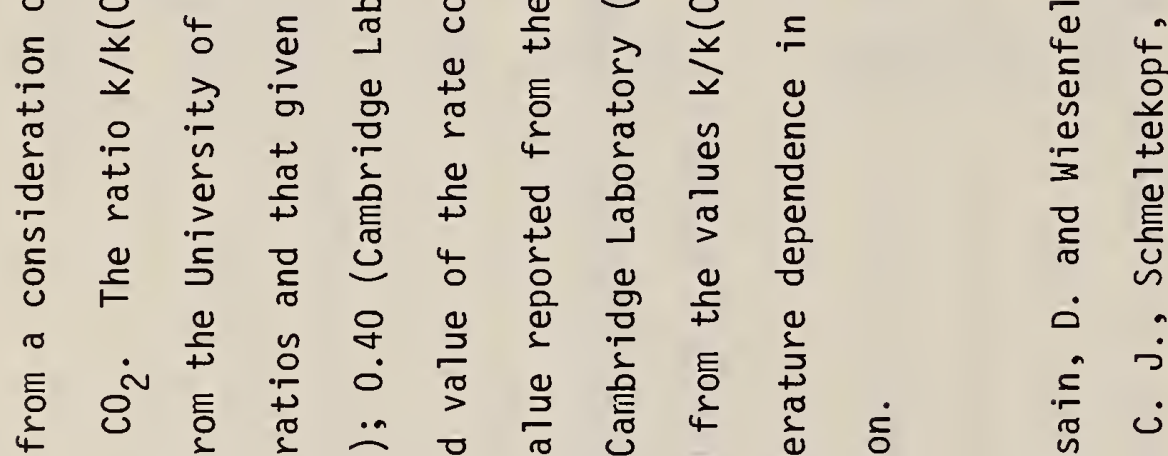

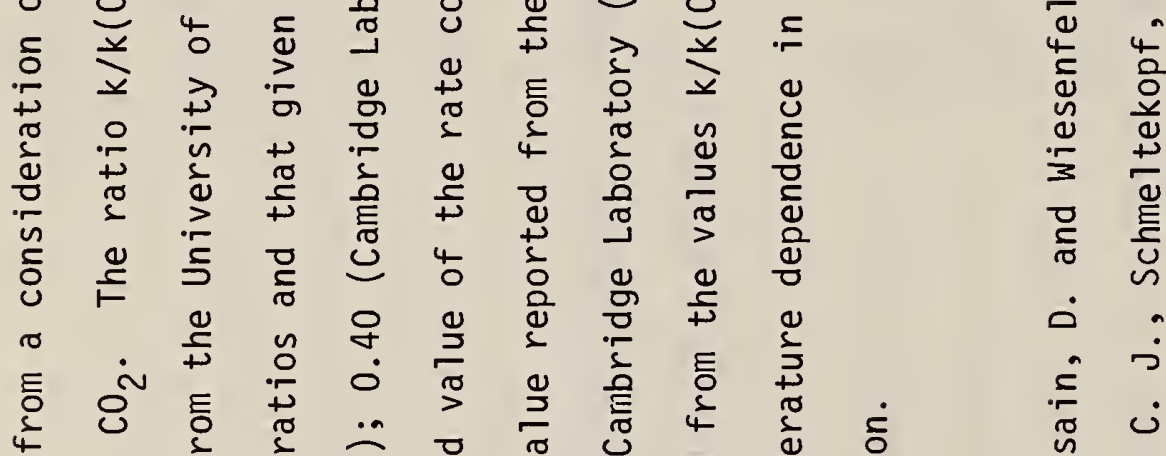

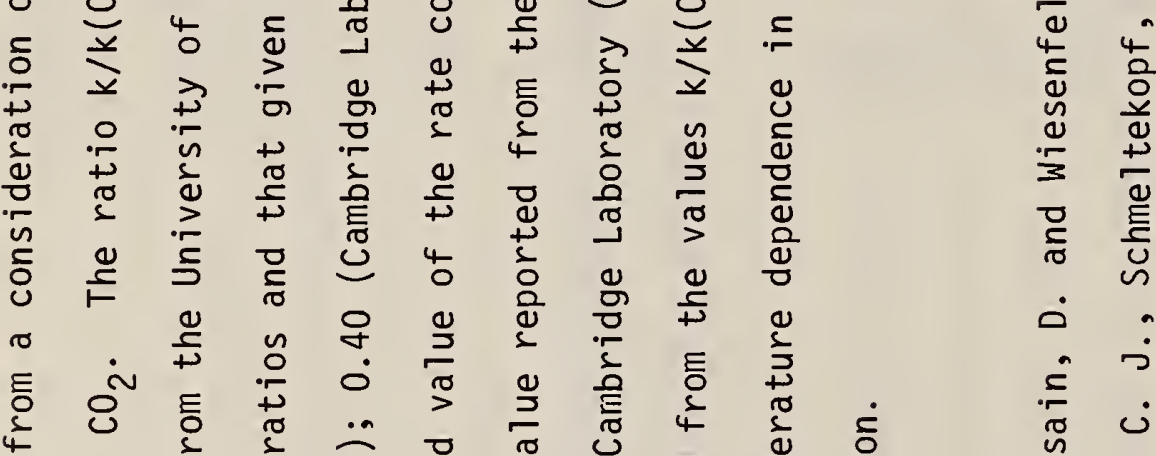

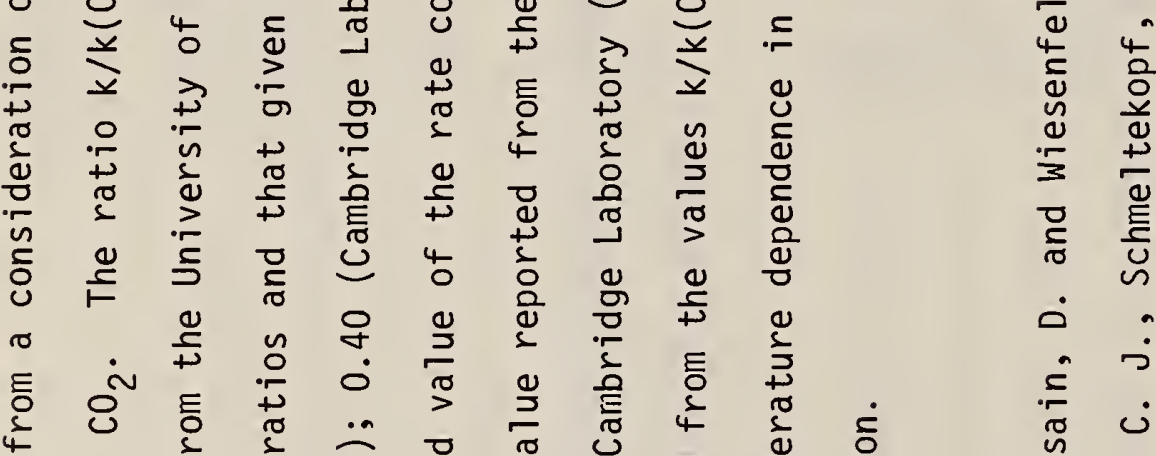

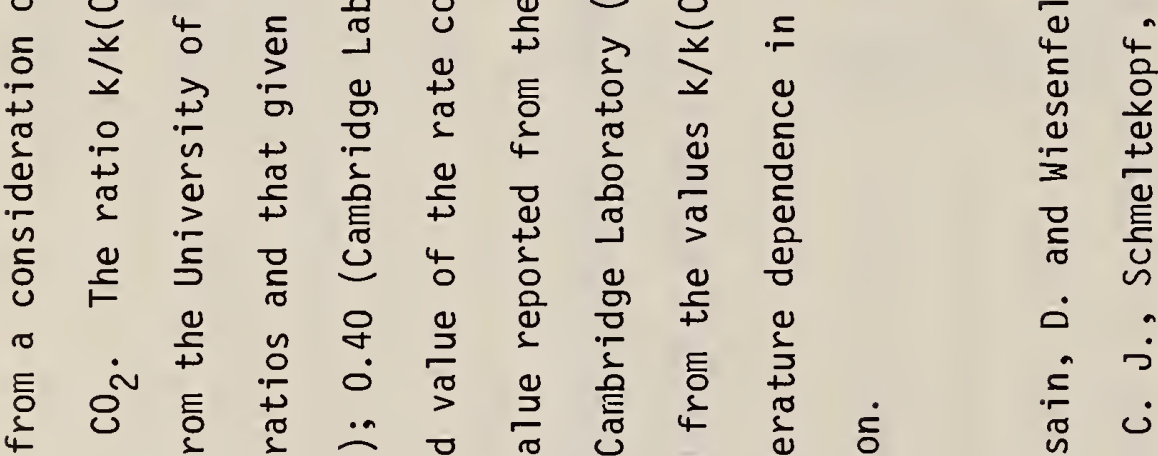

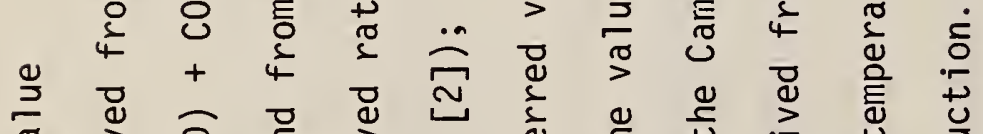

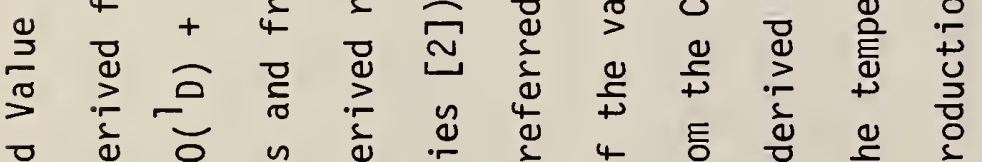

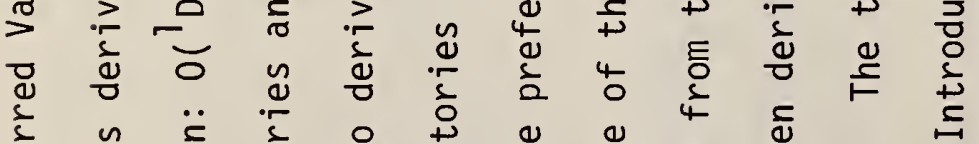

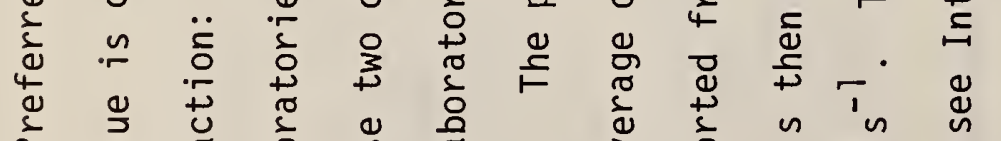

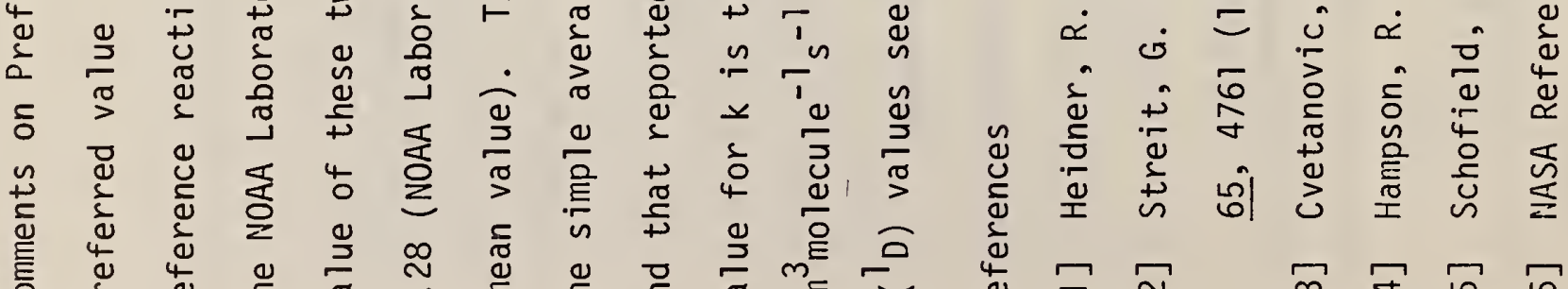

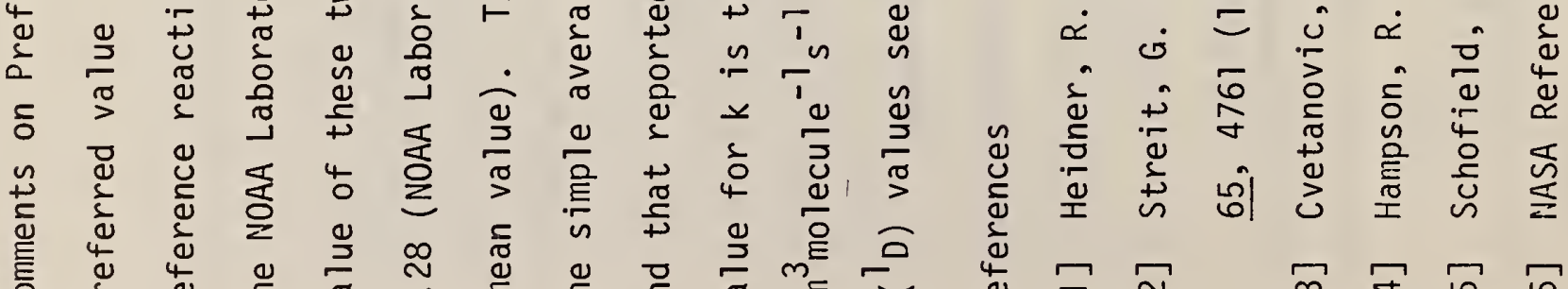

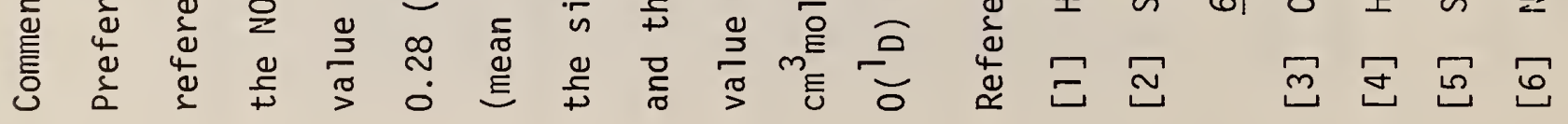




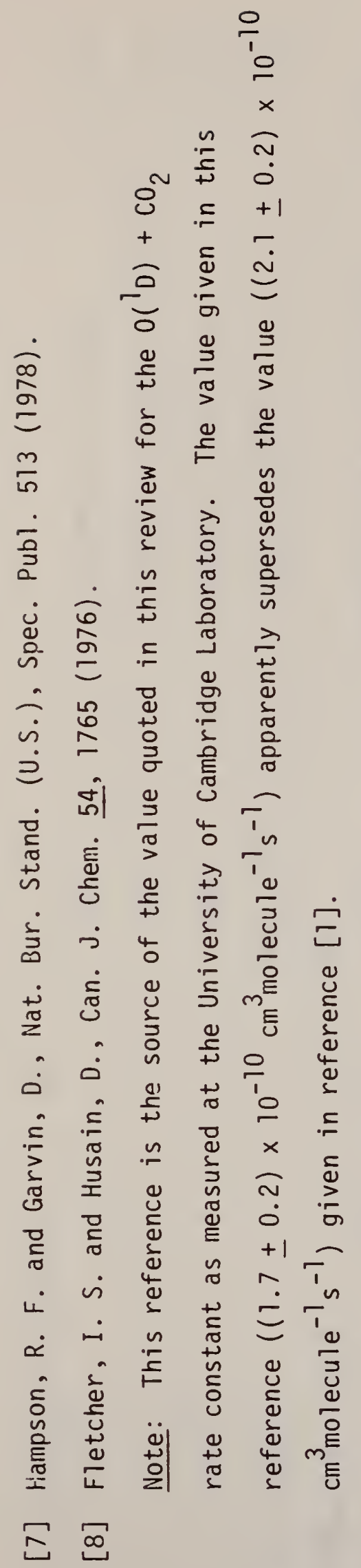




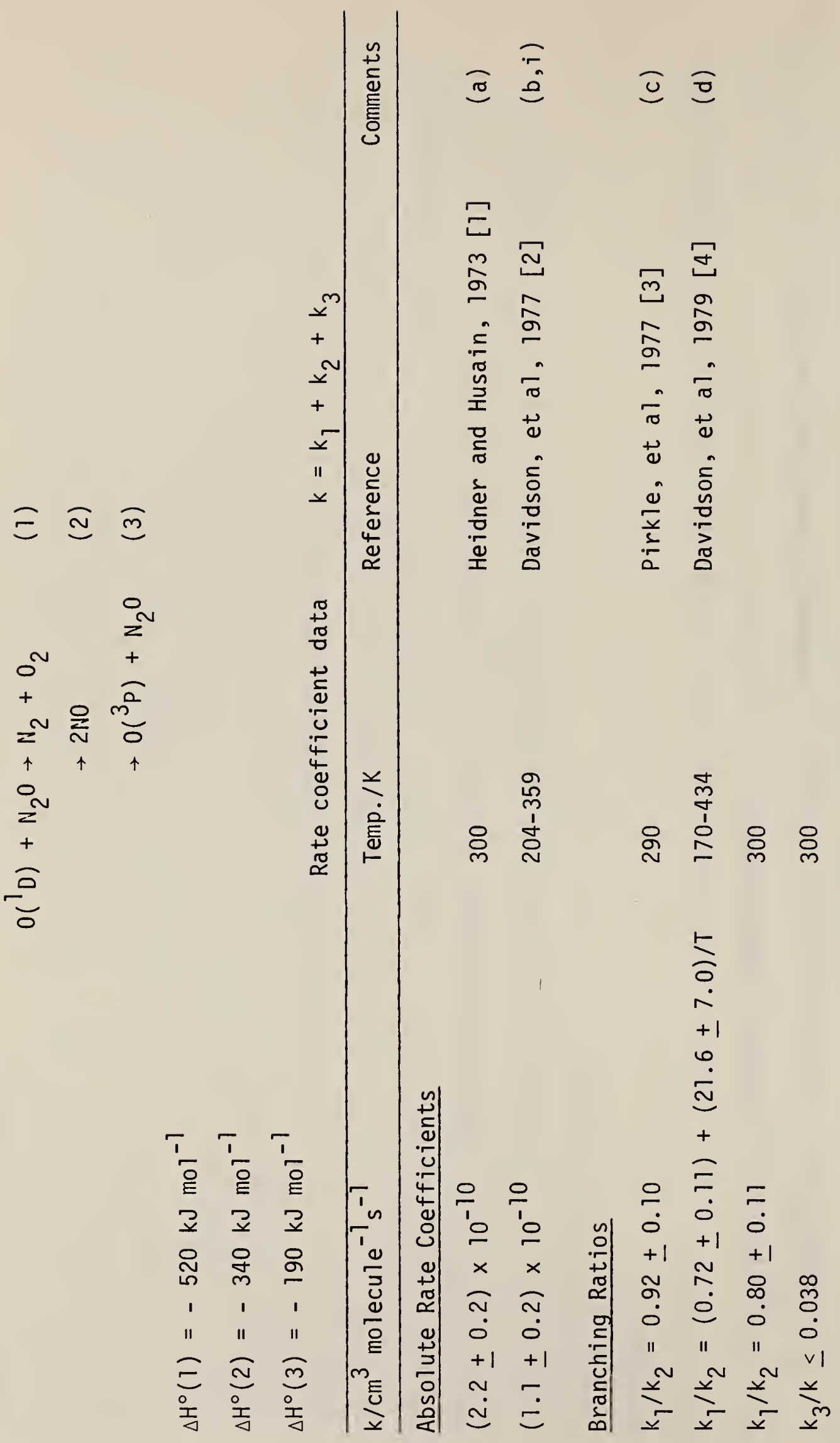




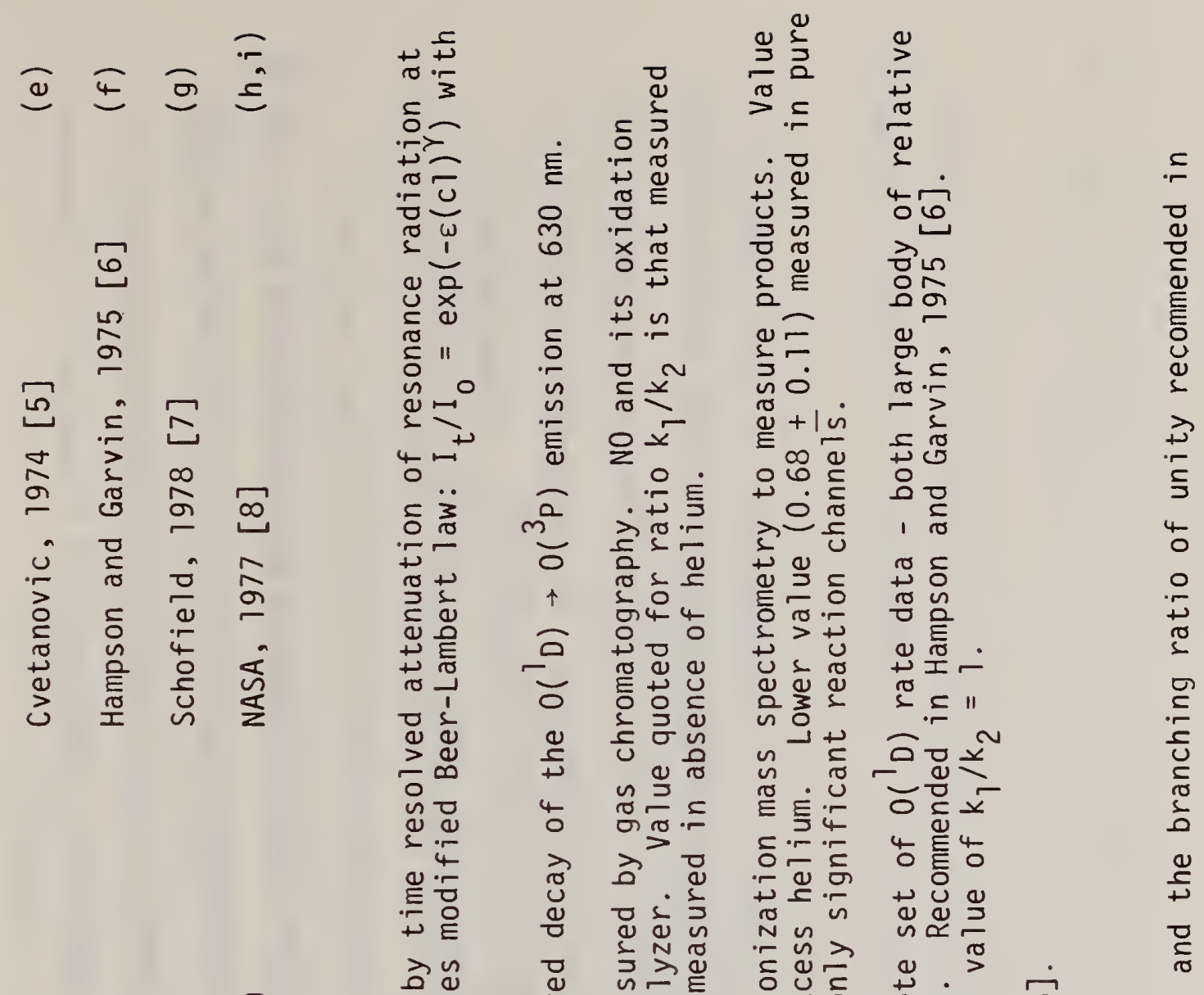

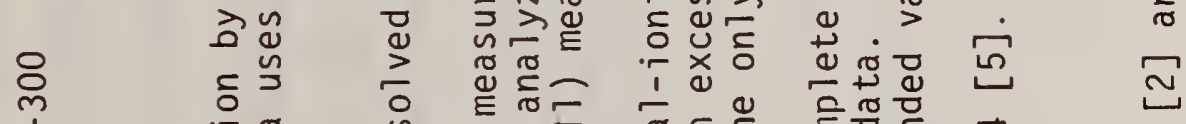

양 욤 品

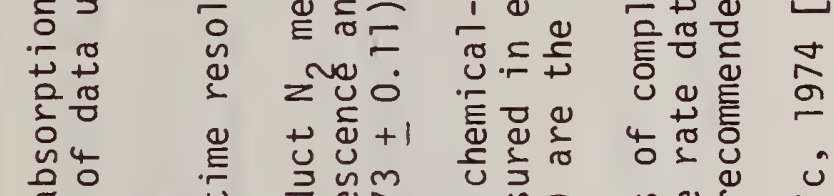

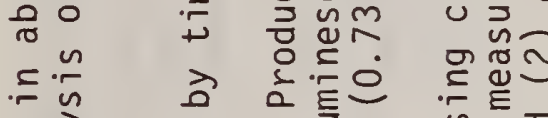

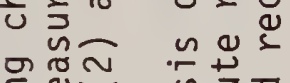

$\approx \cong$ D व

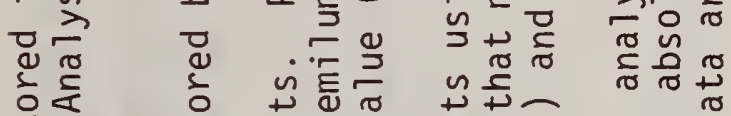

우 운

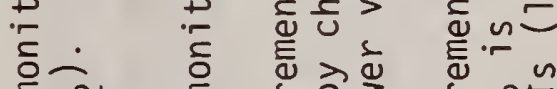

ह

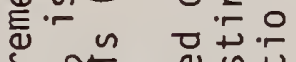

ปे

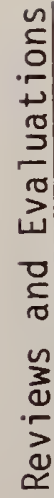

ㅁำ ㄱำ

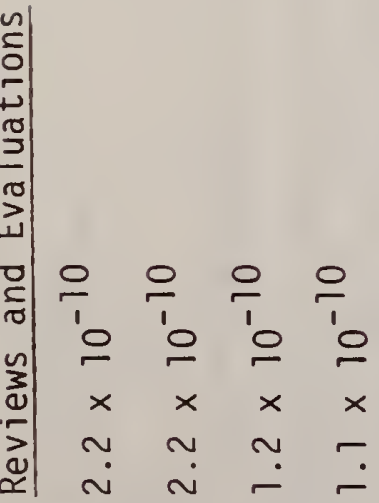

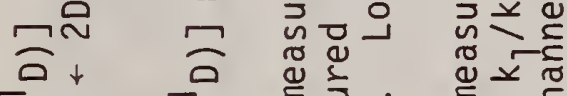

ñ

ริ่

ه व

要过

రํำ 인 유

हं

.

tน

$>$ 든

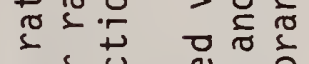

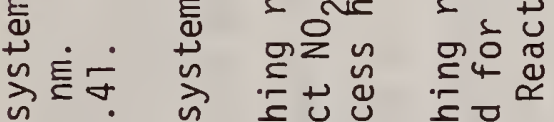

궁

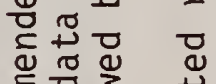

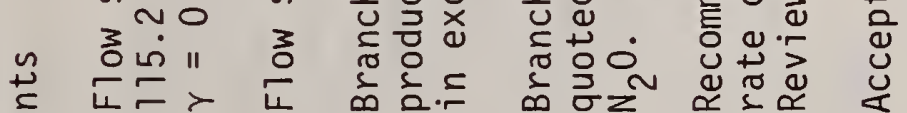

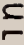

造

¿

4

$\stackrel{0}{+}$

$\frac{9}{c}$
$\frac{0}{0}$
$\frac{5}{0}$
$\frac{1}{2}$
$\frac{1}{4}$

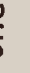




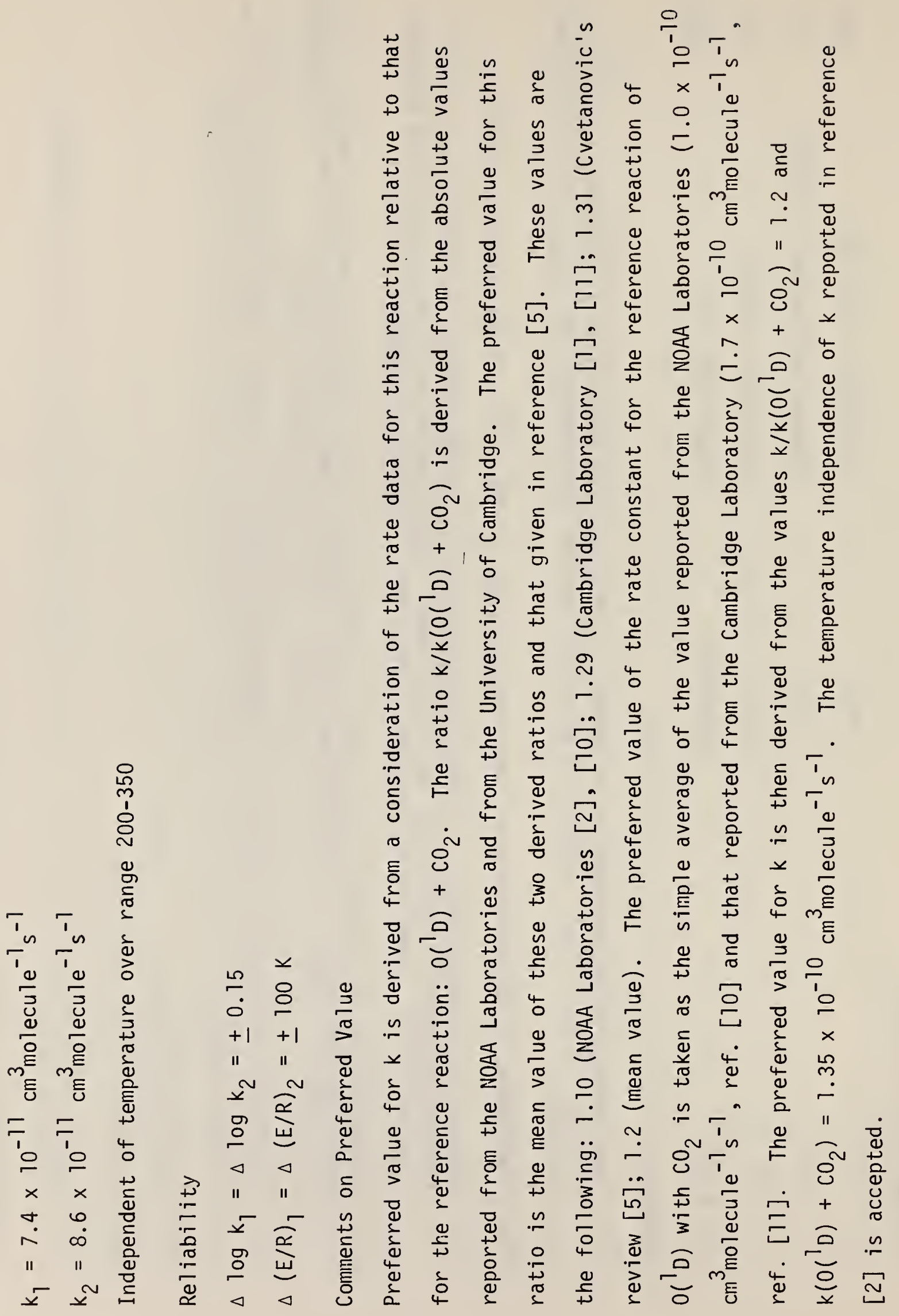




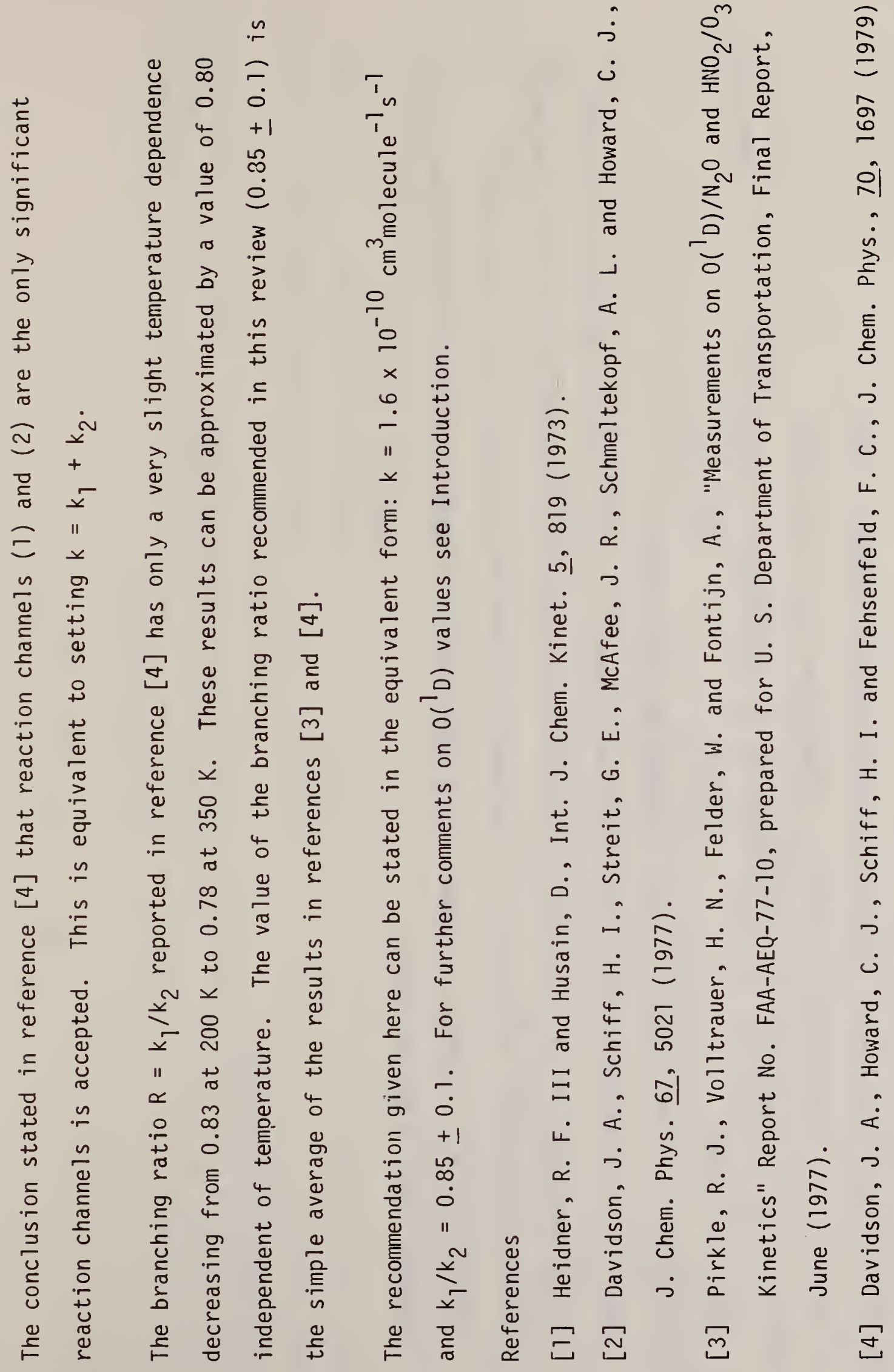




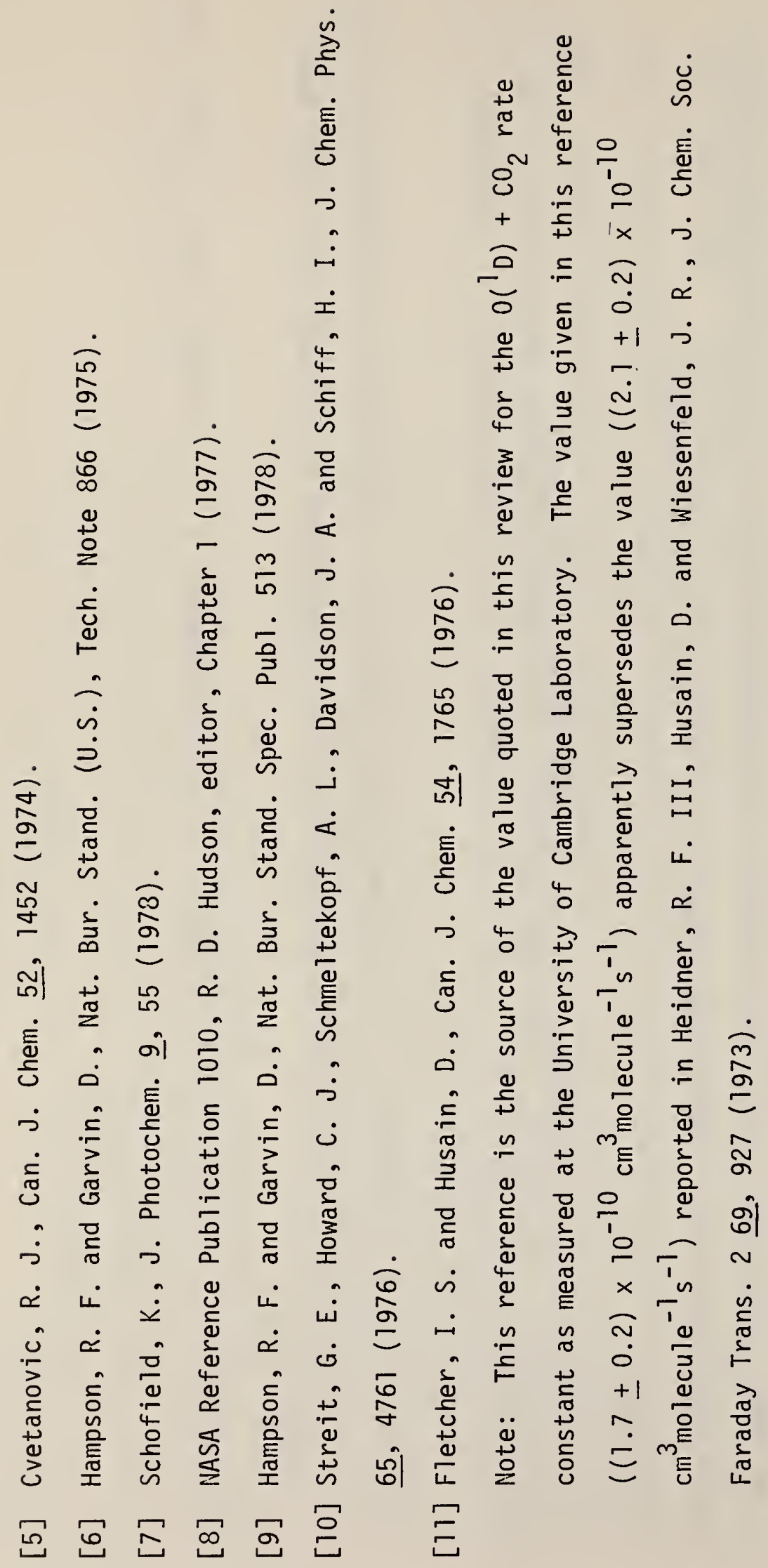




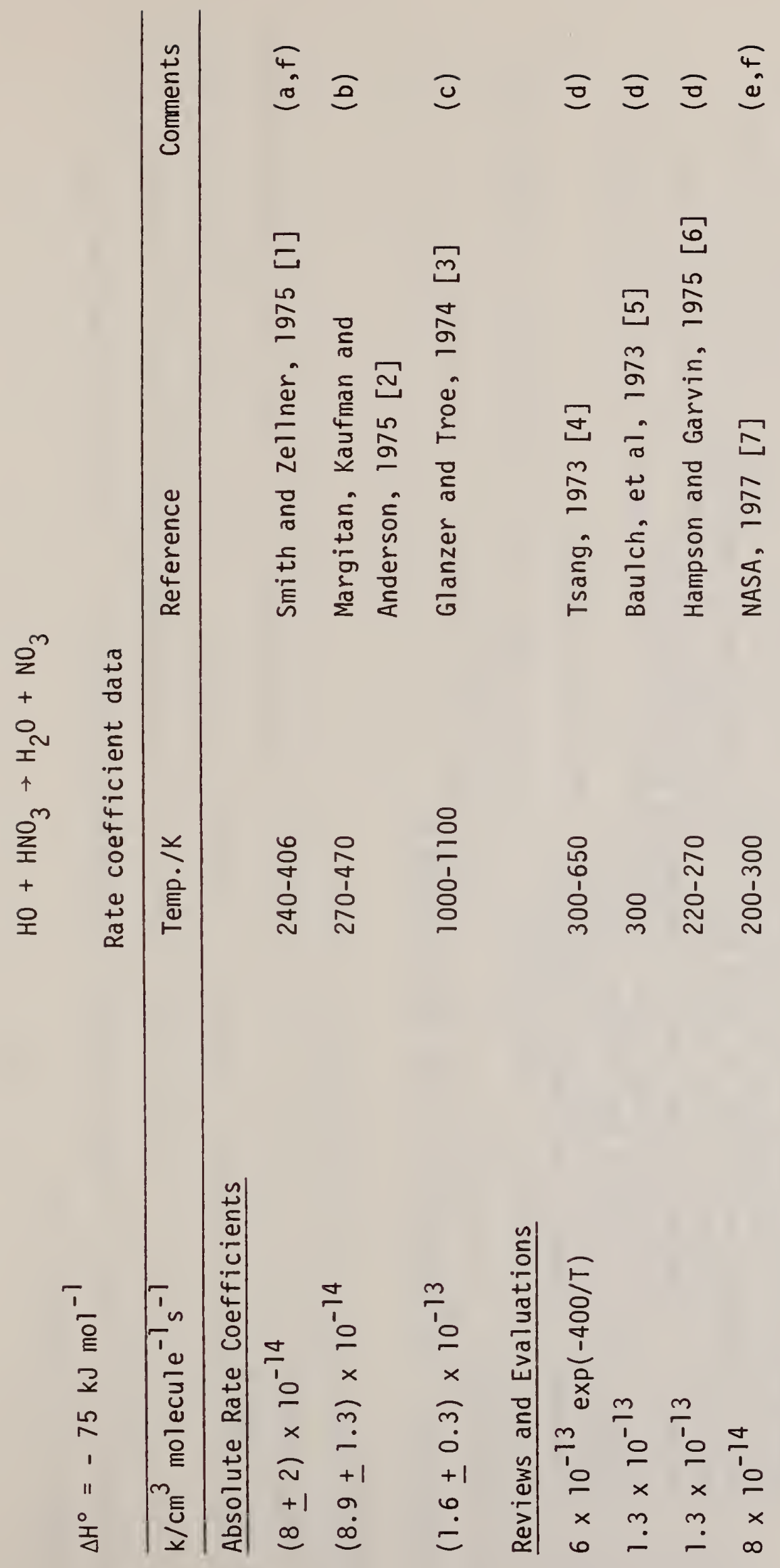




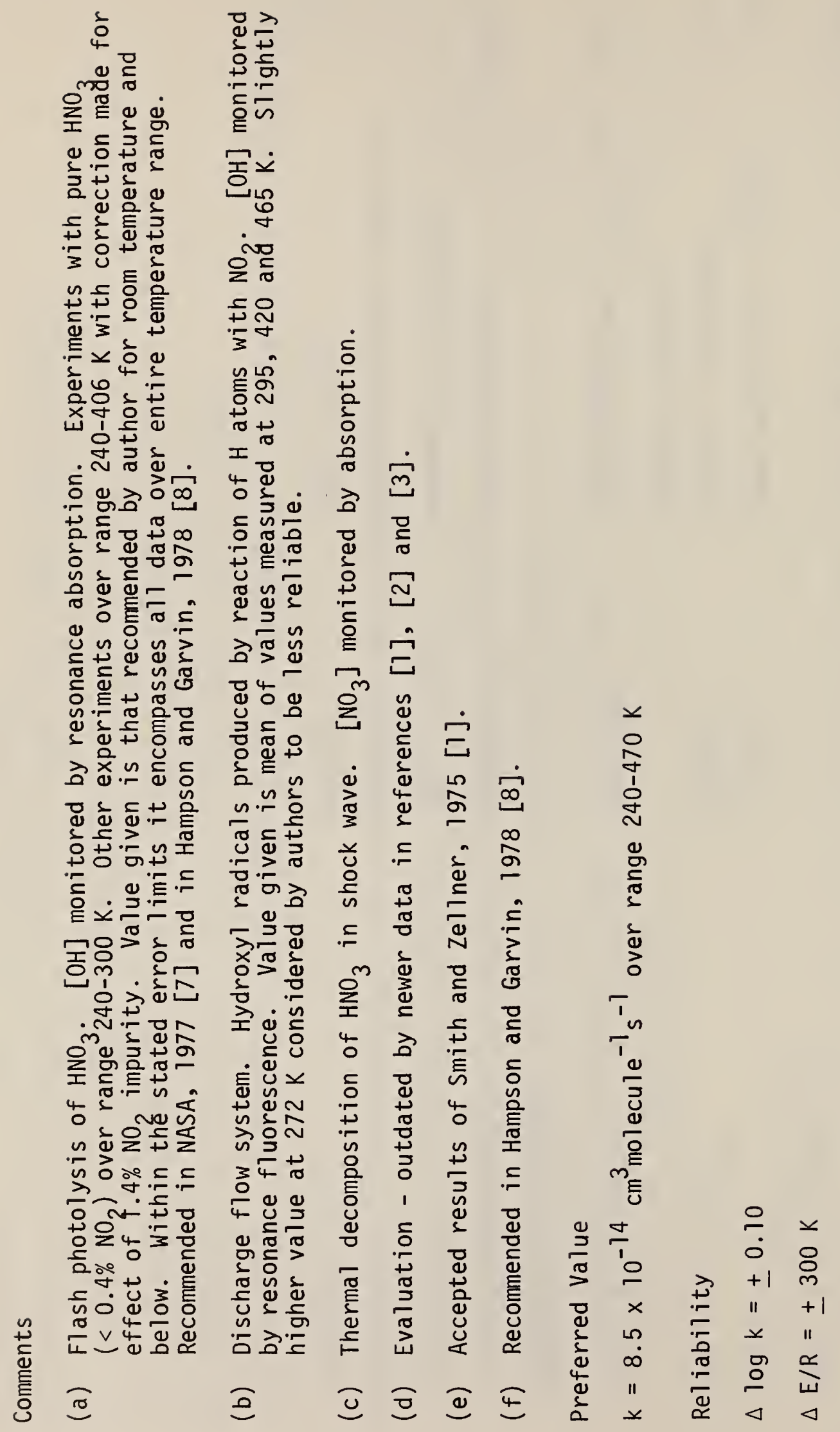




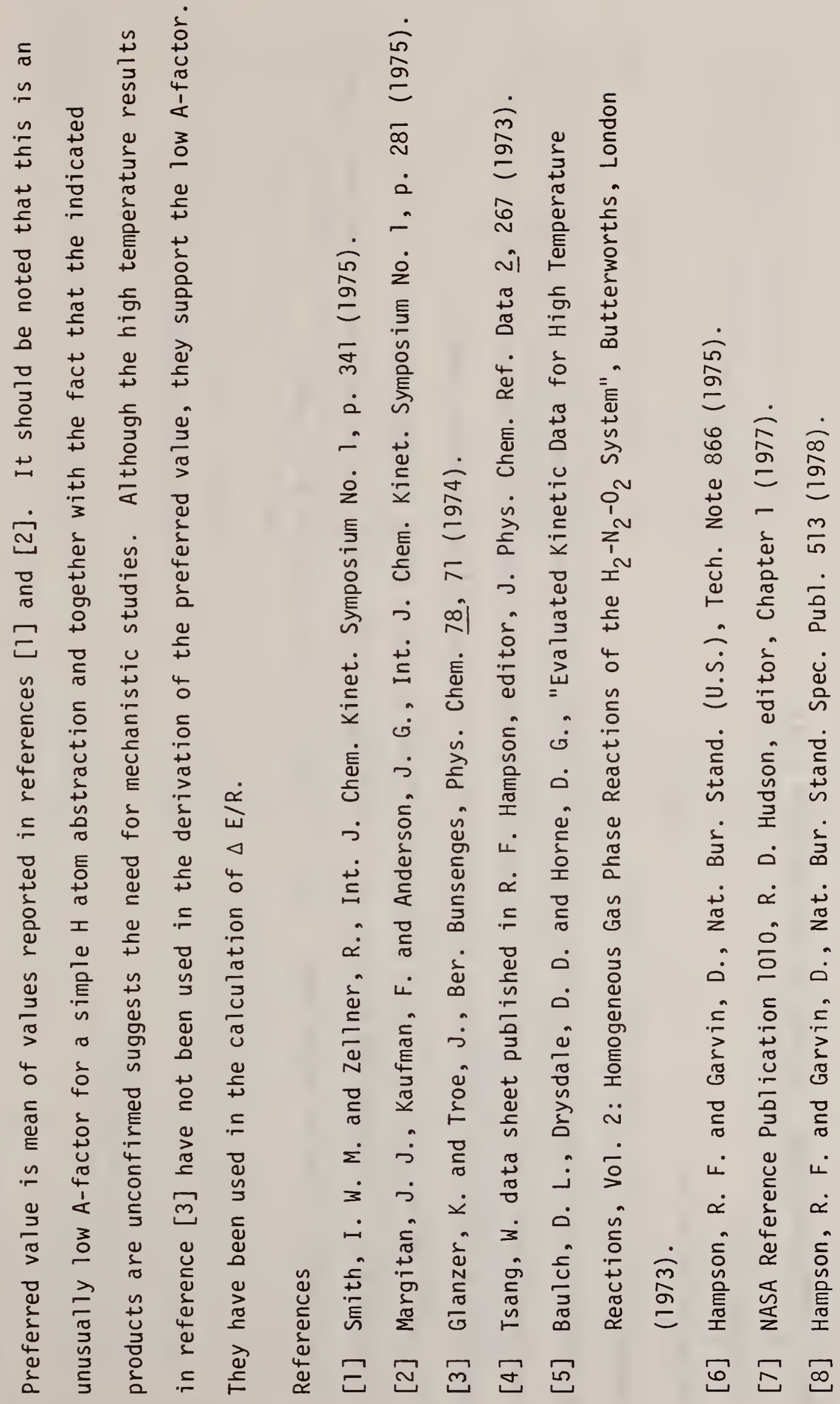




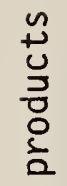

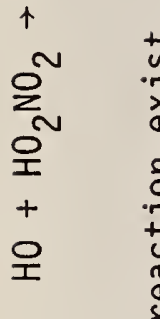




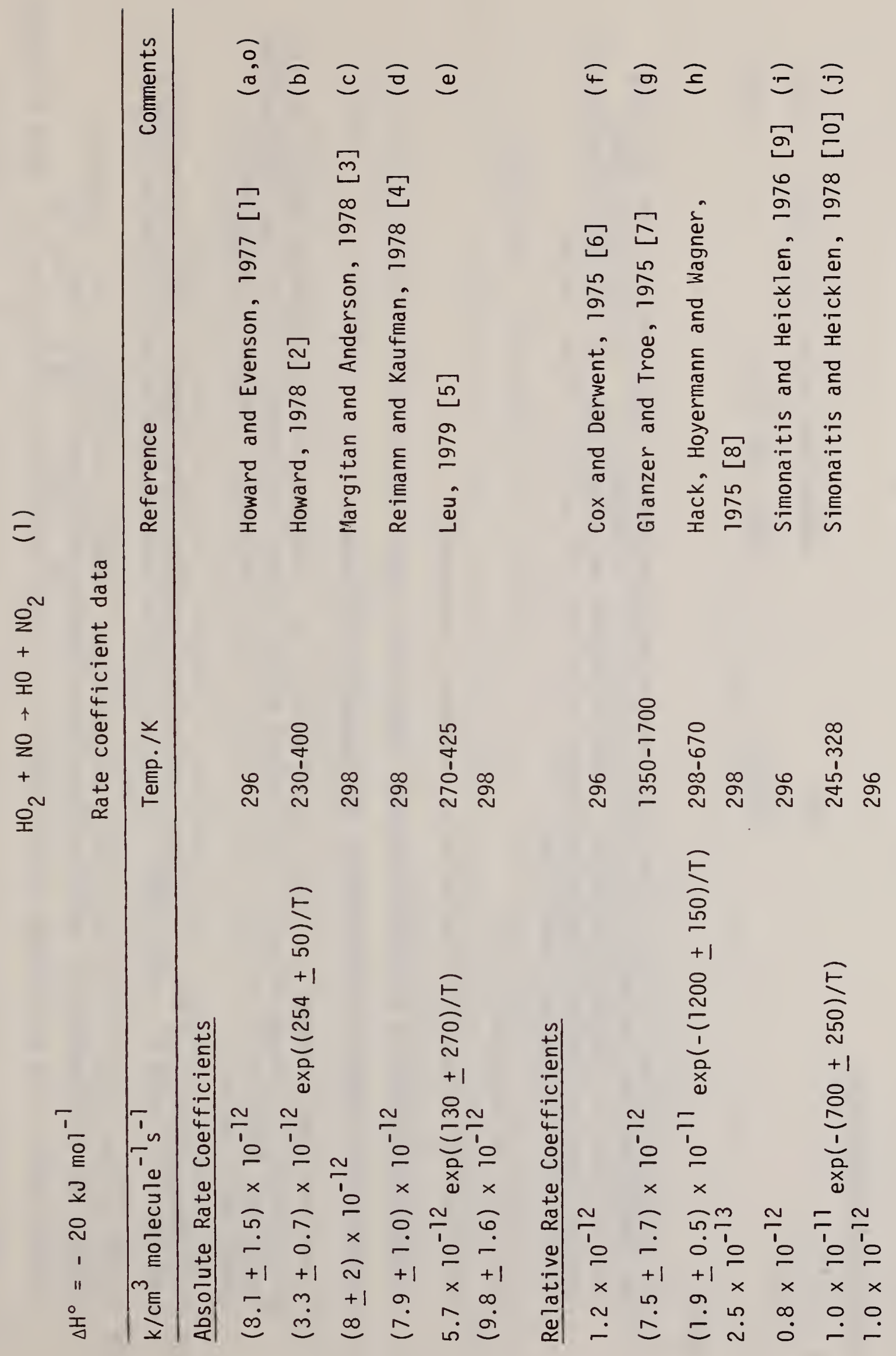




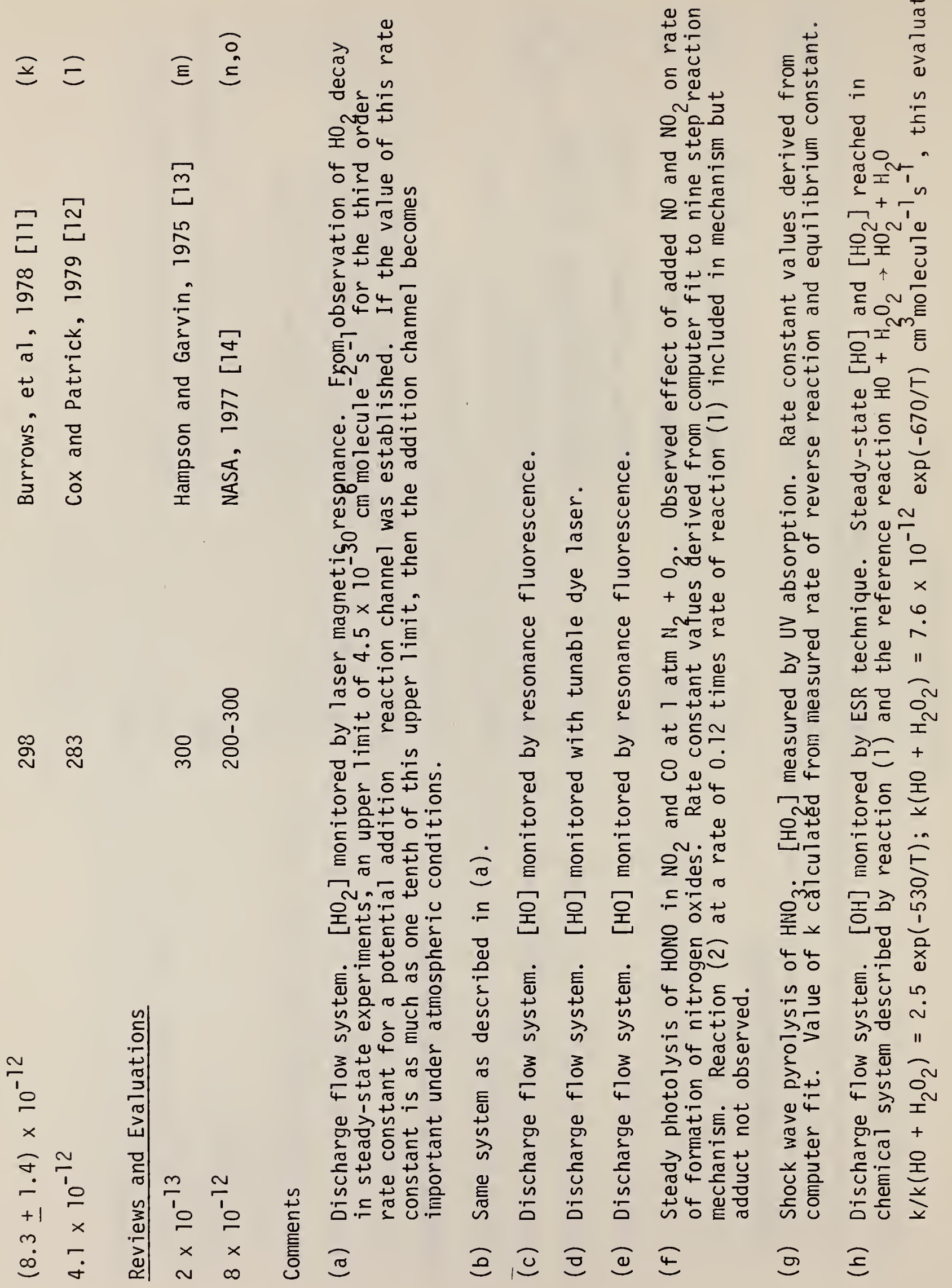




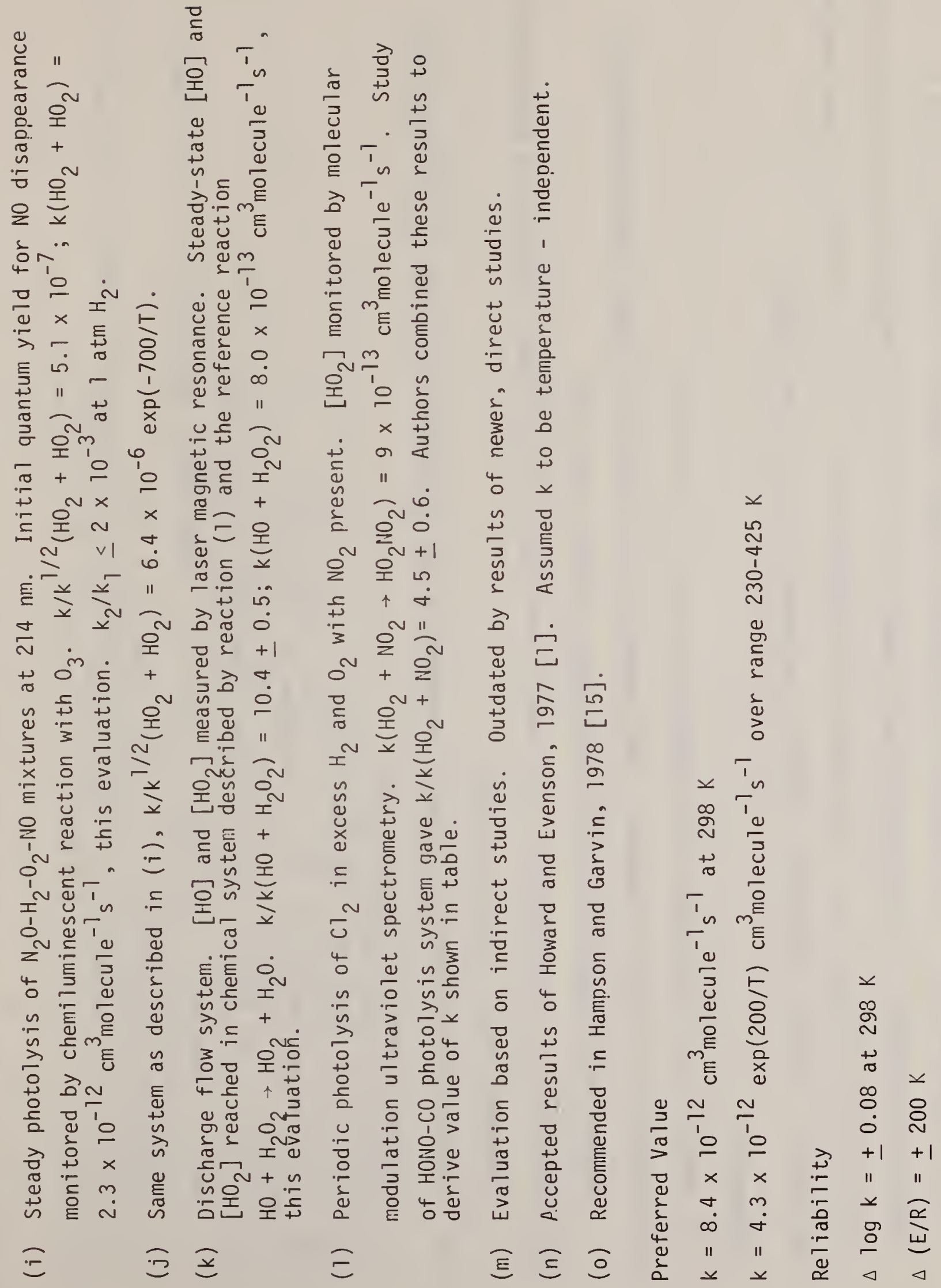




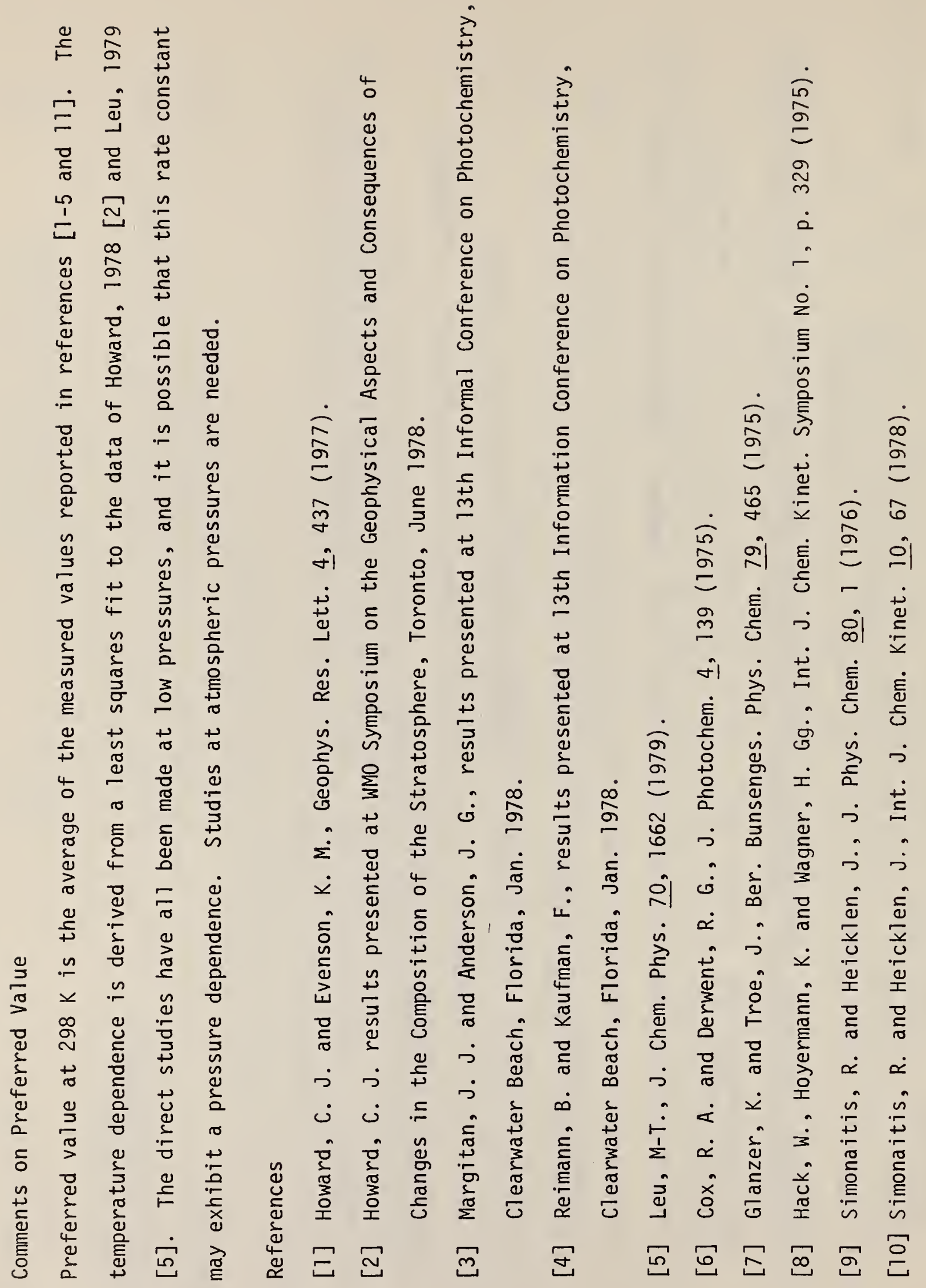




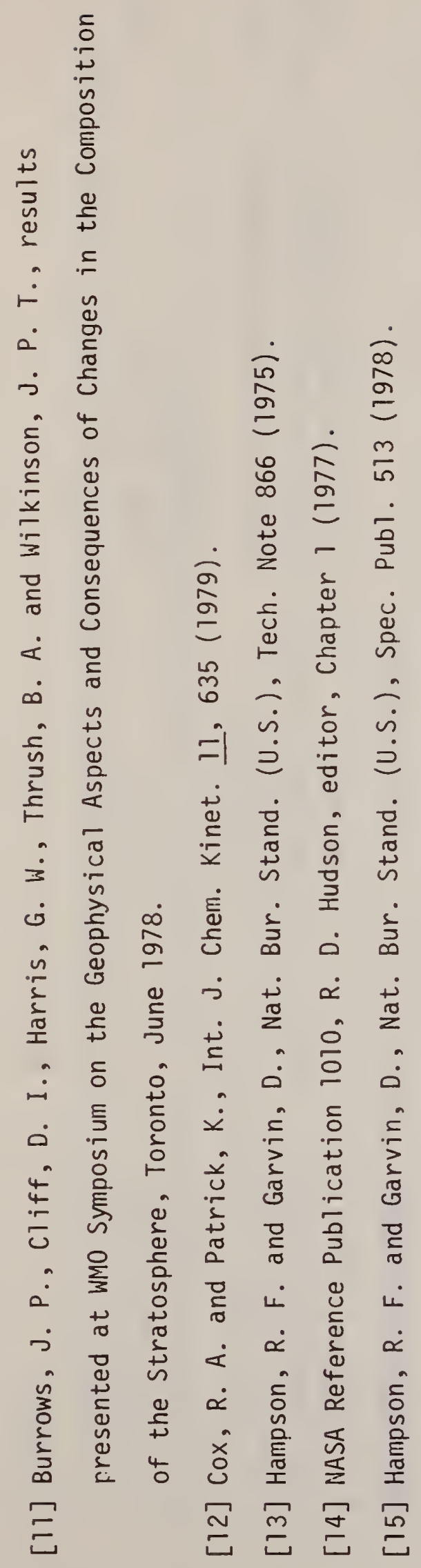




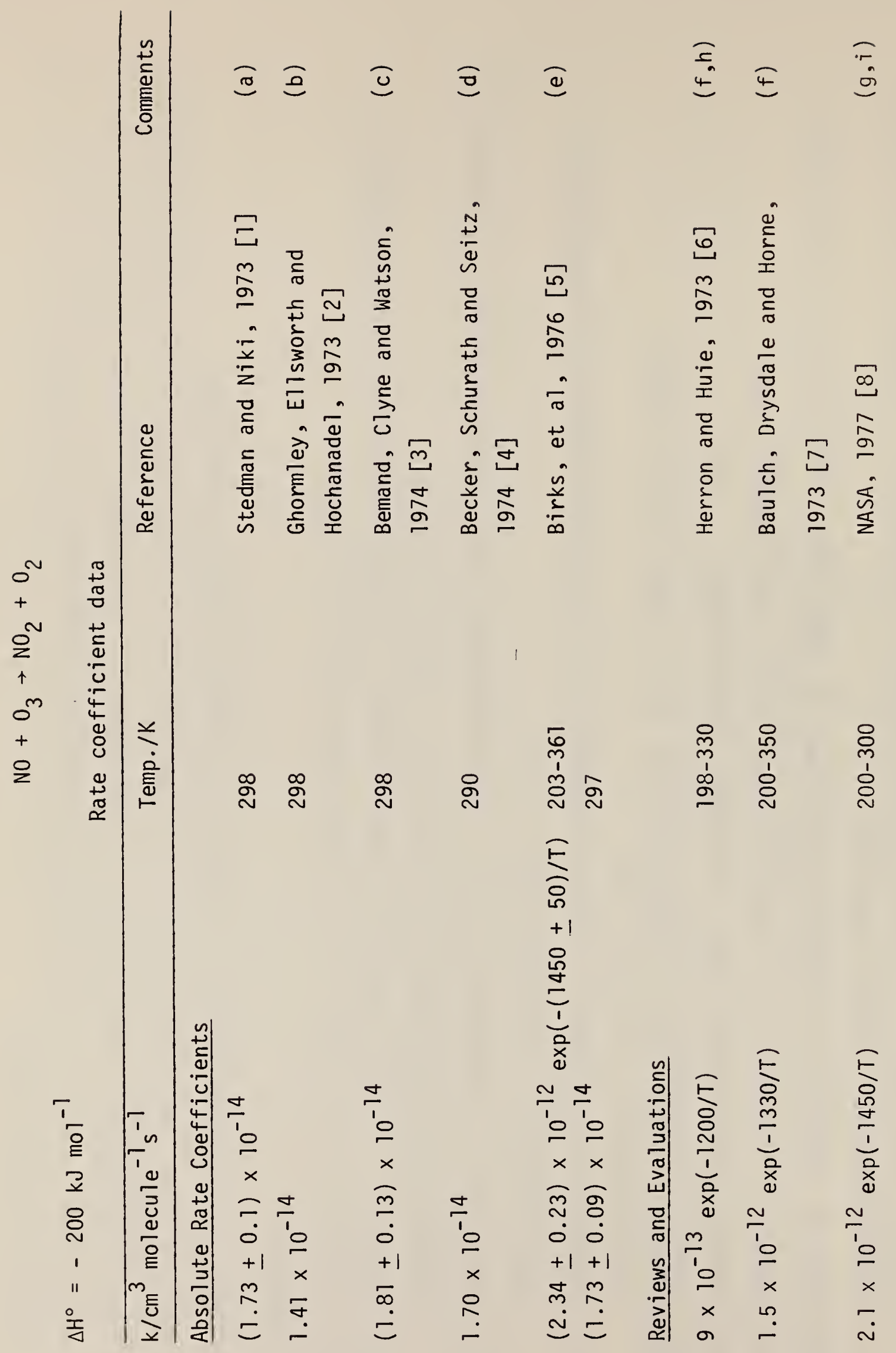




$$
\begin{aligned}
& \text { 苞 } \\
& \text { ֻั๊ }
\end{aligned}
$$

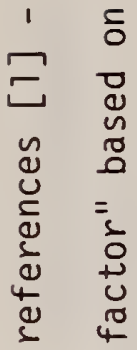

$$
\begin{aligned}
& \text { a } \therefore \leqq
\end{aligned}
$$

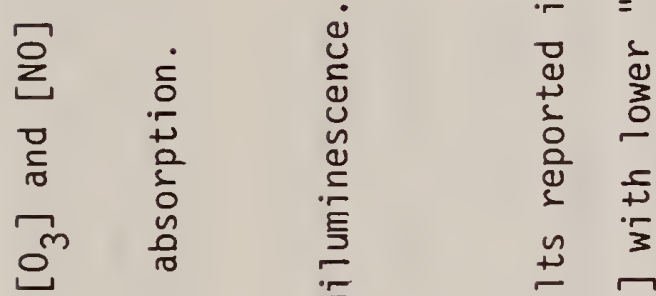

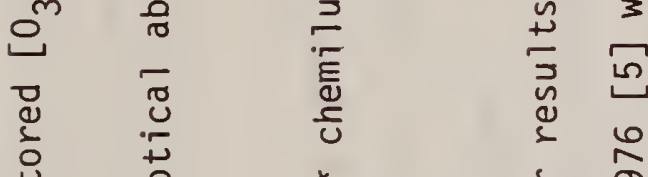

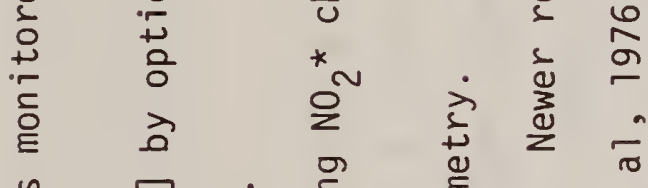

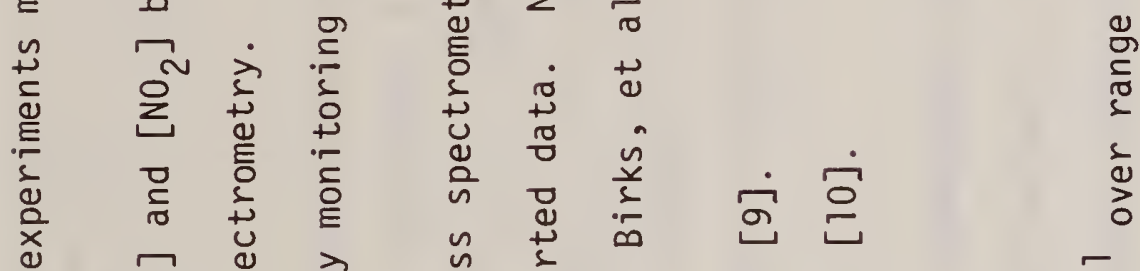

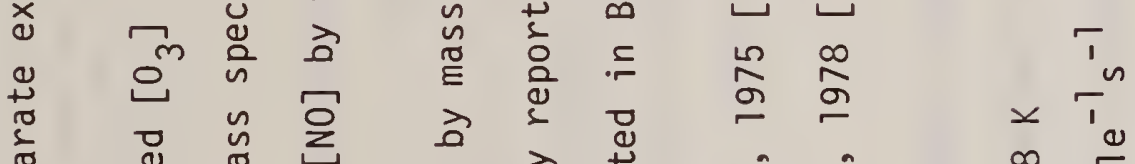

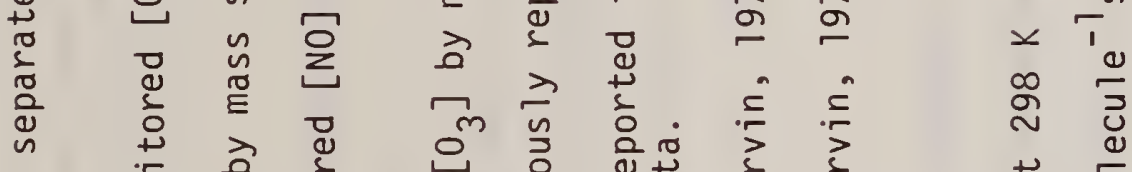

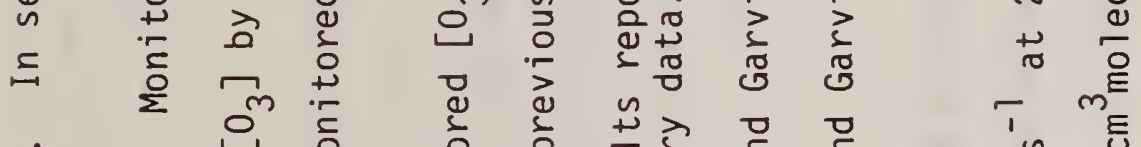

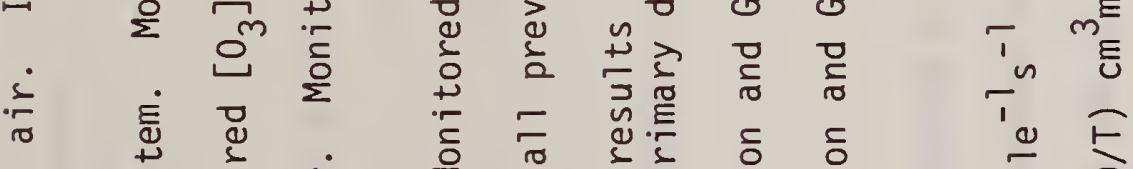

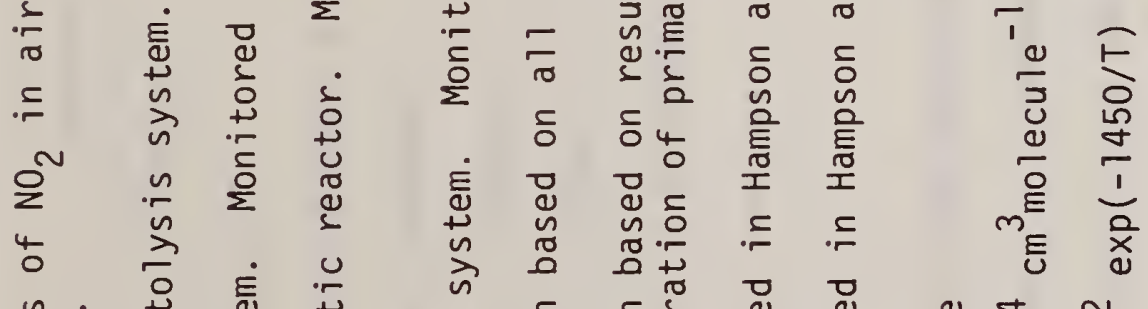

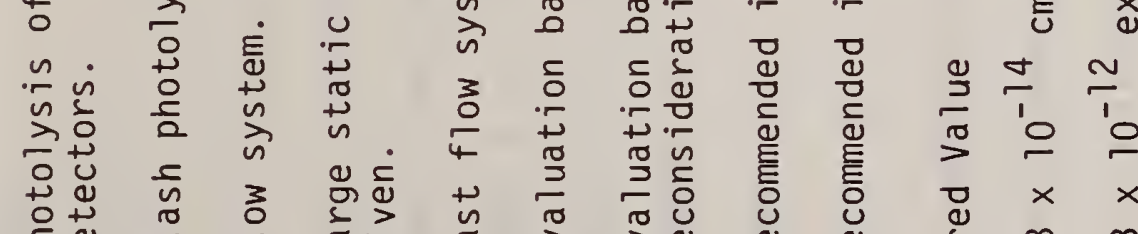

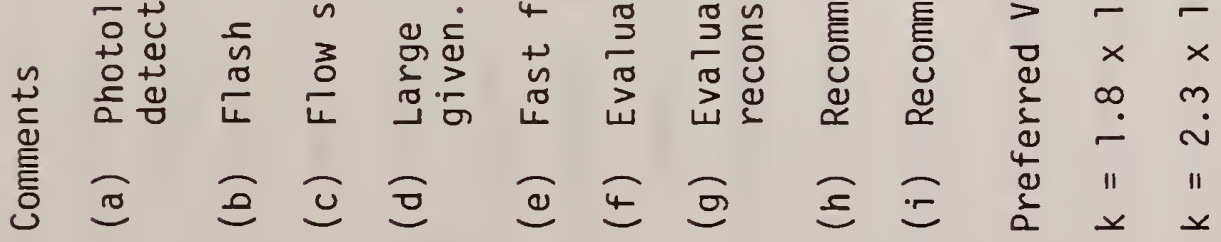




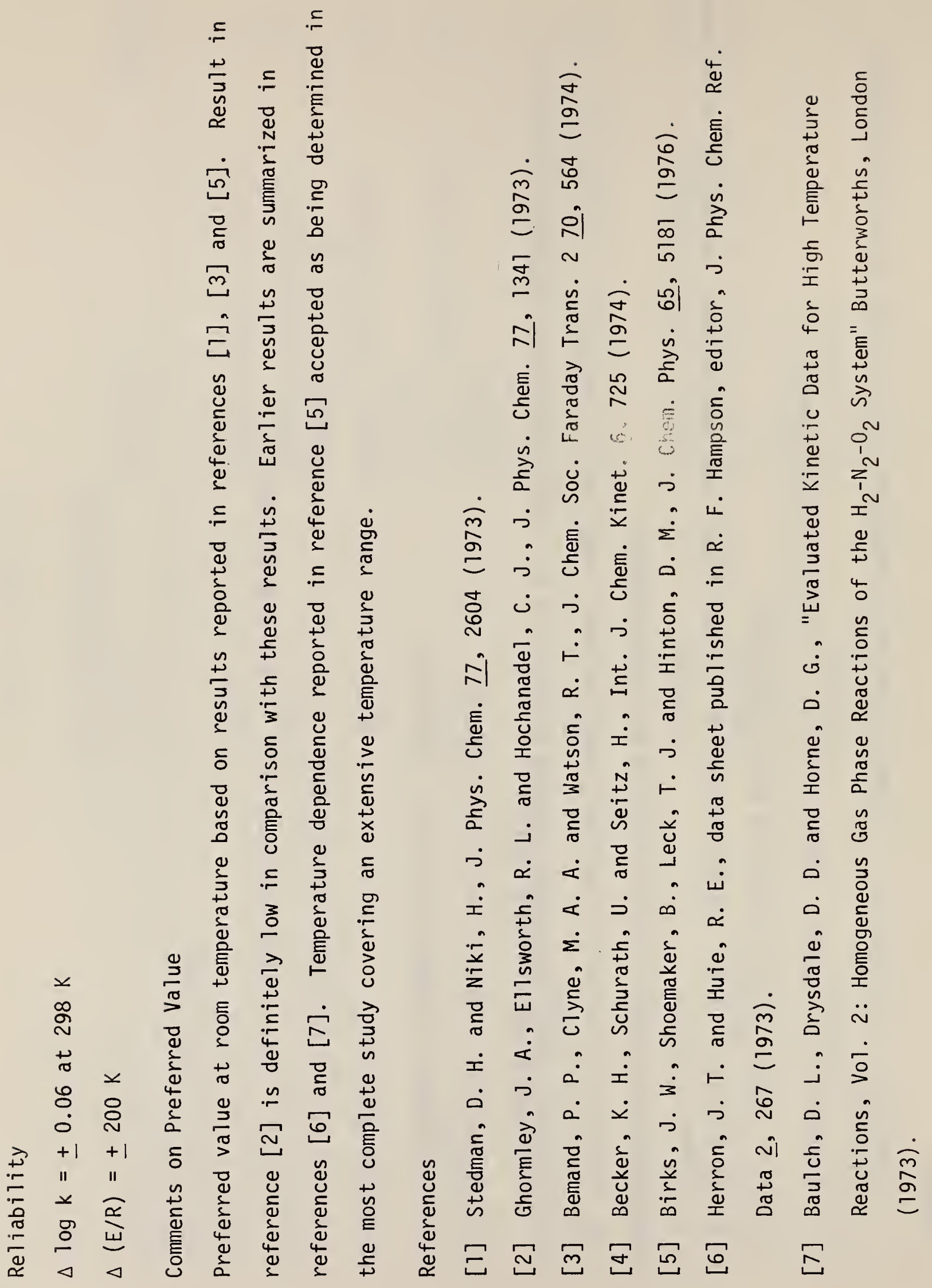




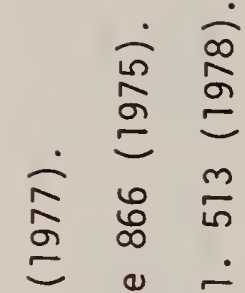

$$
\begin{aligned}
& \text { 雚 } \\
& \text { 离 } \\
& \text { के } \\
& \text { 范 } \dot{2}
\end{aligned}
$$

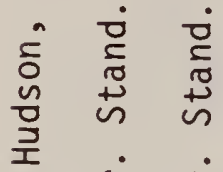

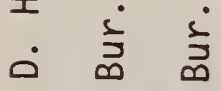

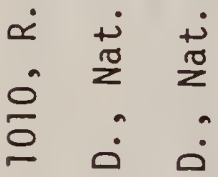

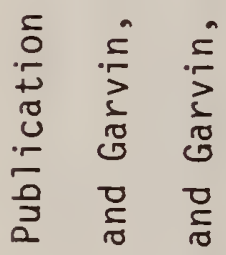

$$
\begin{aligned}
& \stackrel{\Xi}{\check{\Sigma}} \\
& \text { 这 } \propto \\
& \text { ฯ } \\
& \text { 芯長長 } \\
& \text { ๖ Б은 }
\end{aligned}
$$




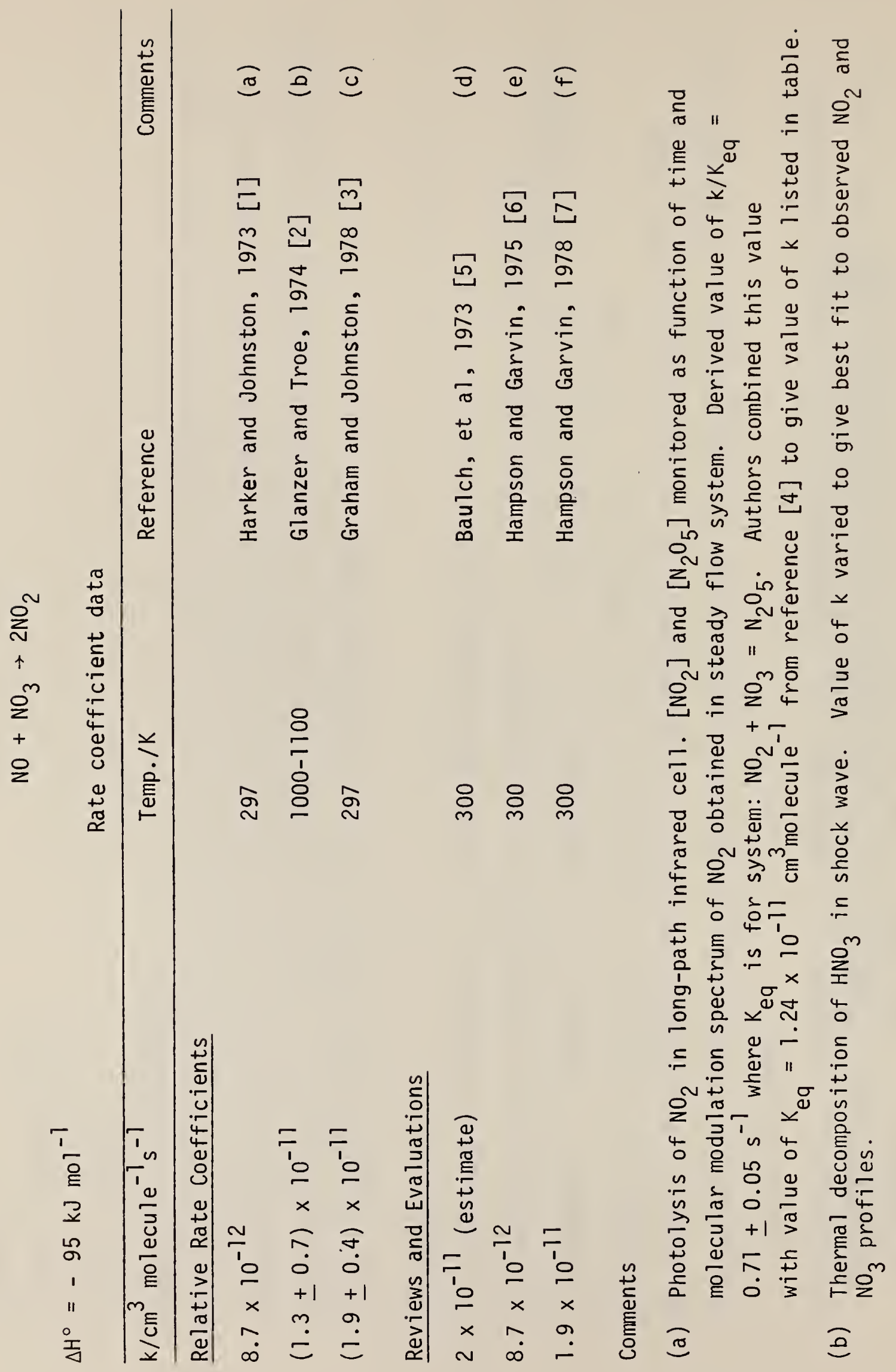




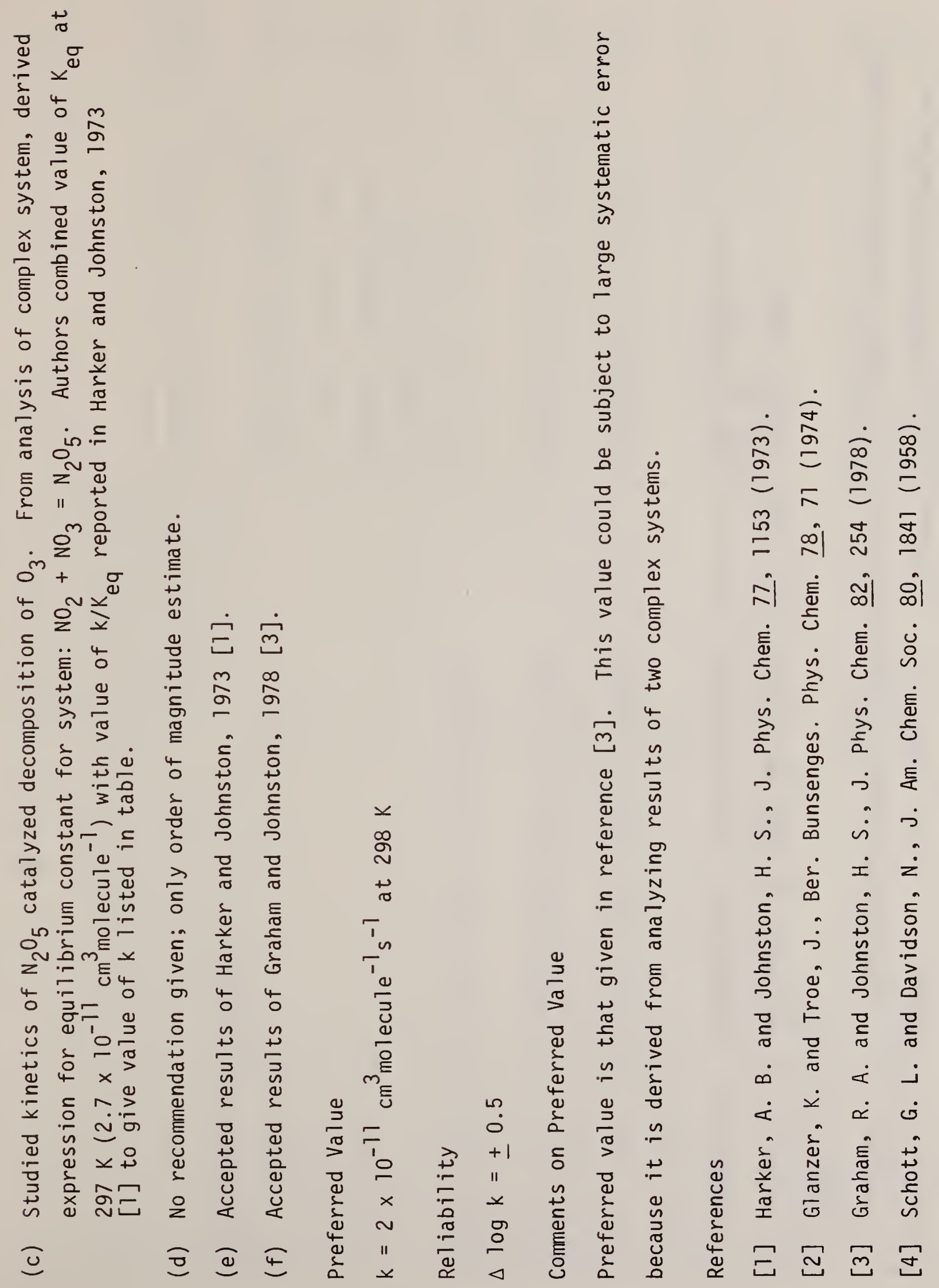




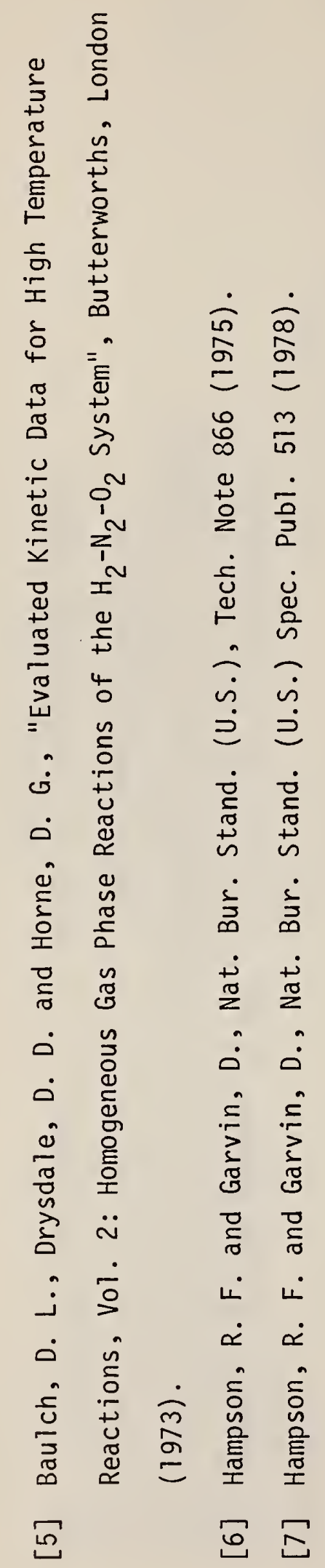




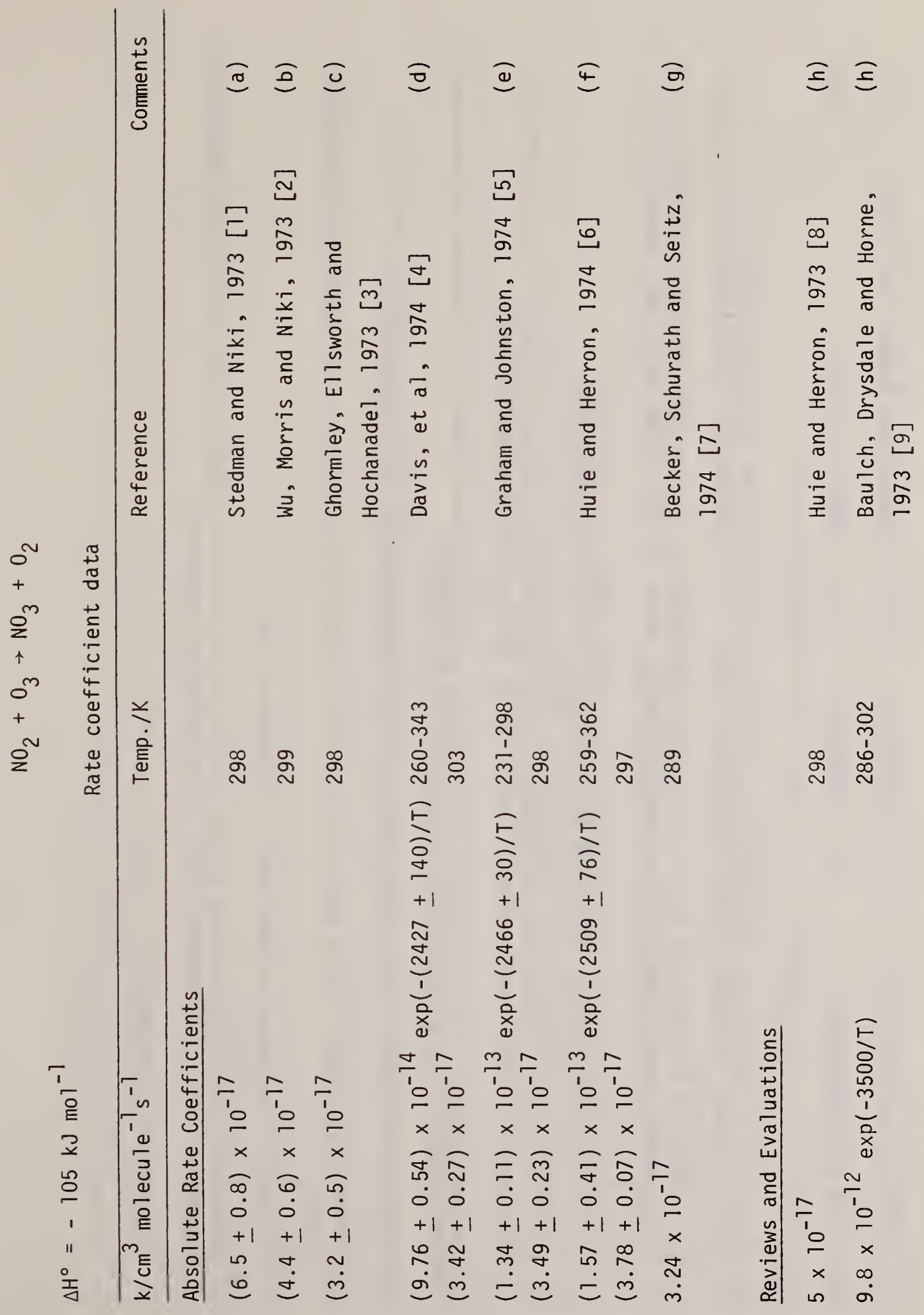




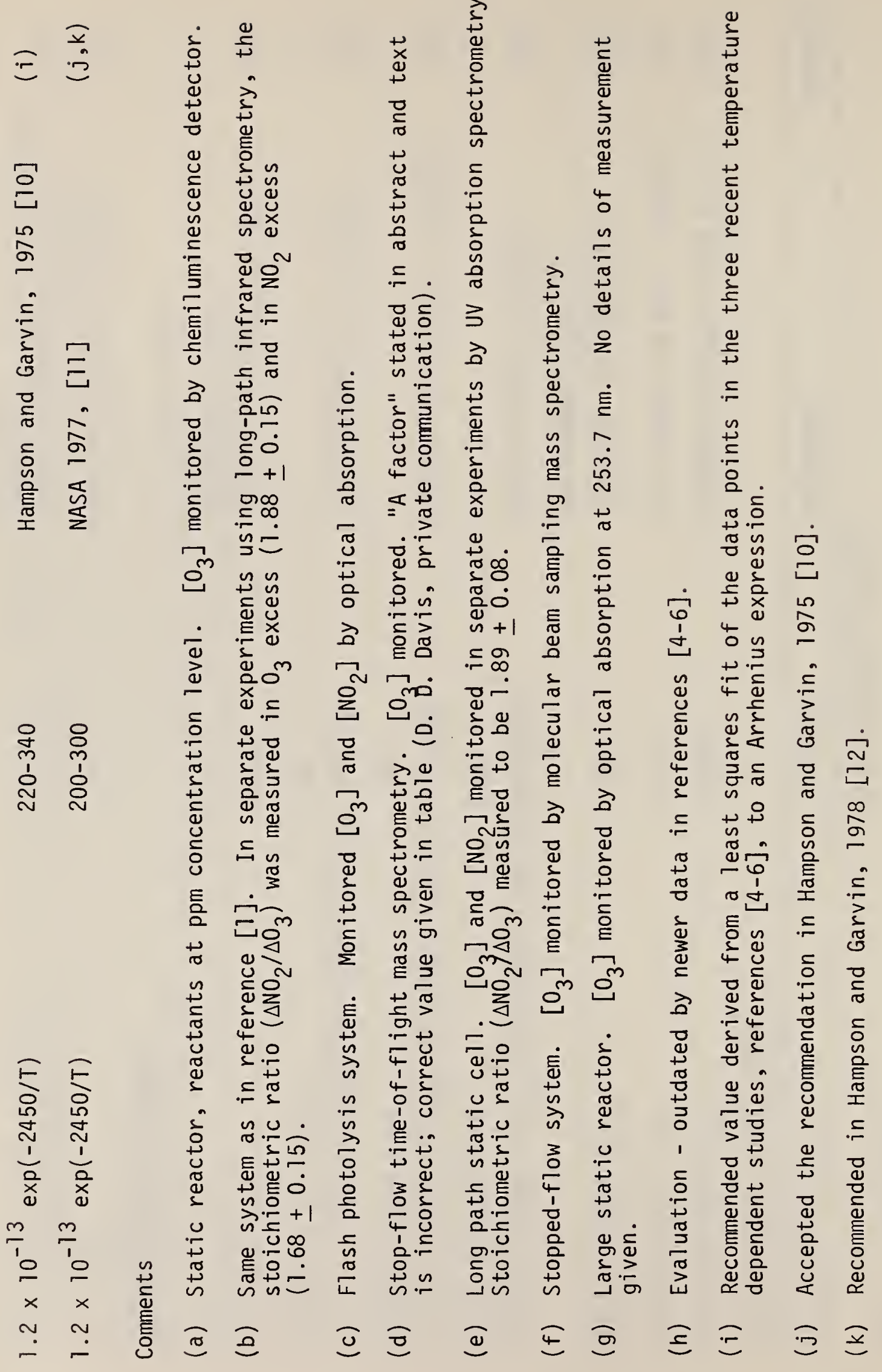




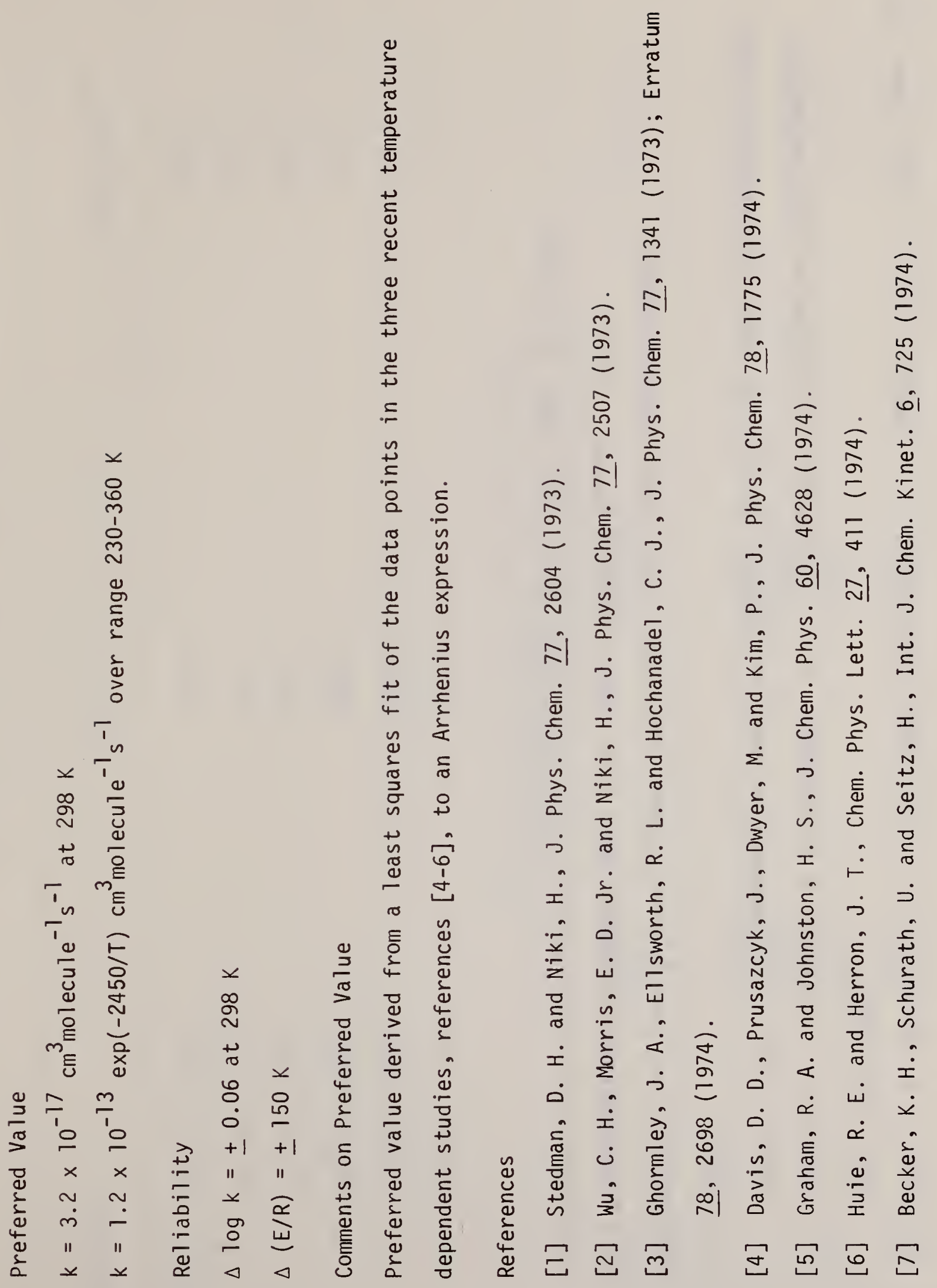




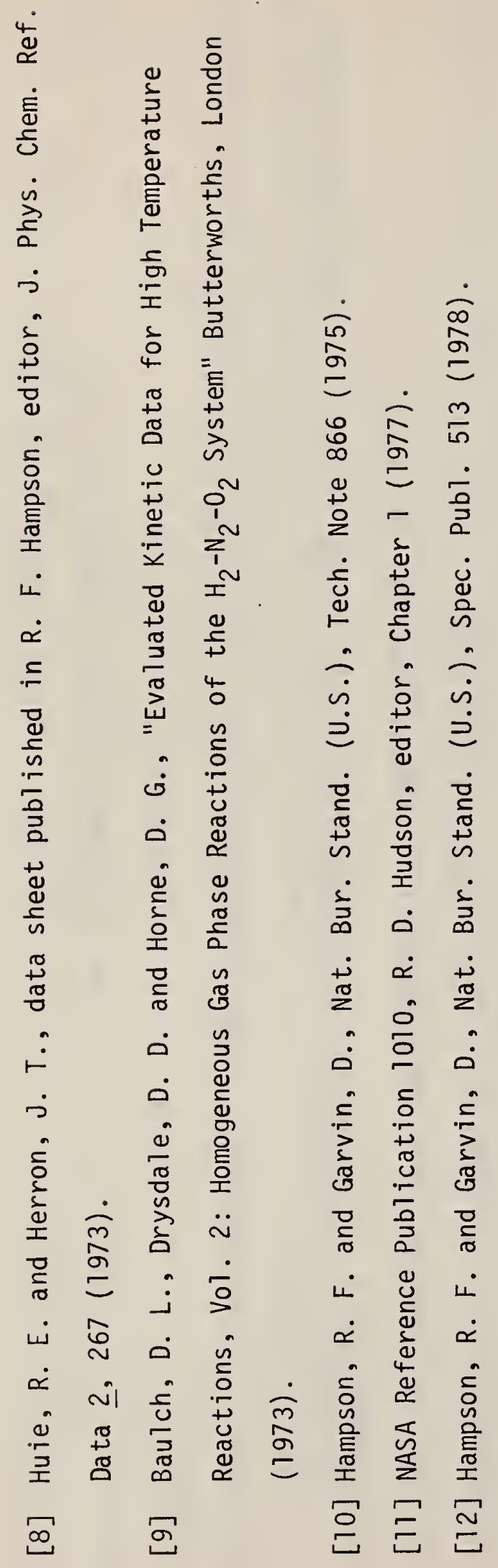




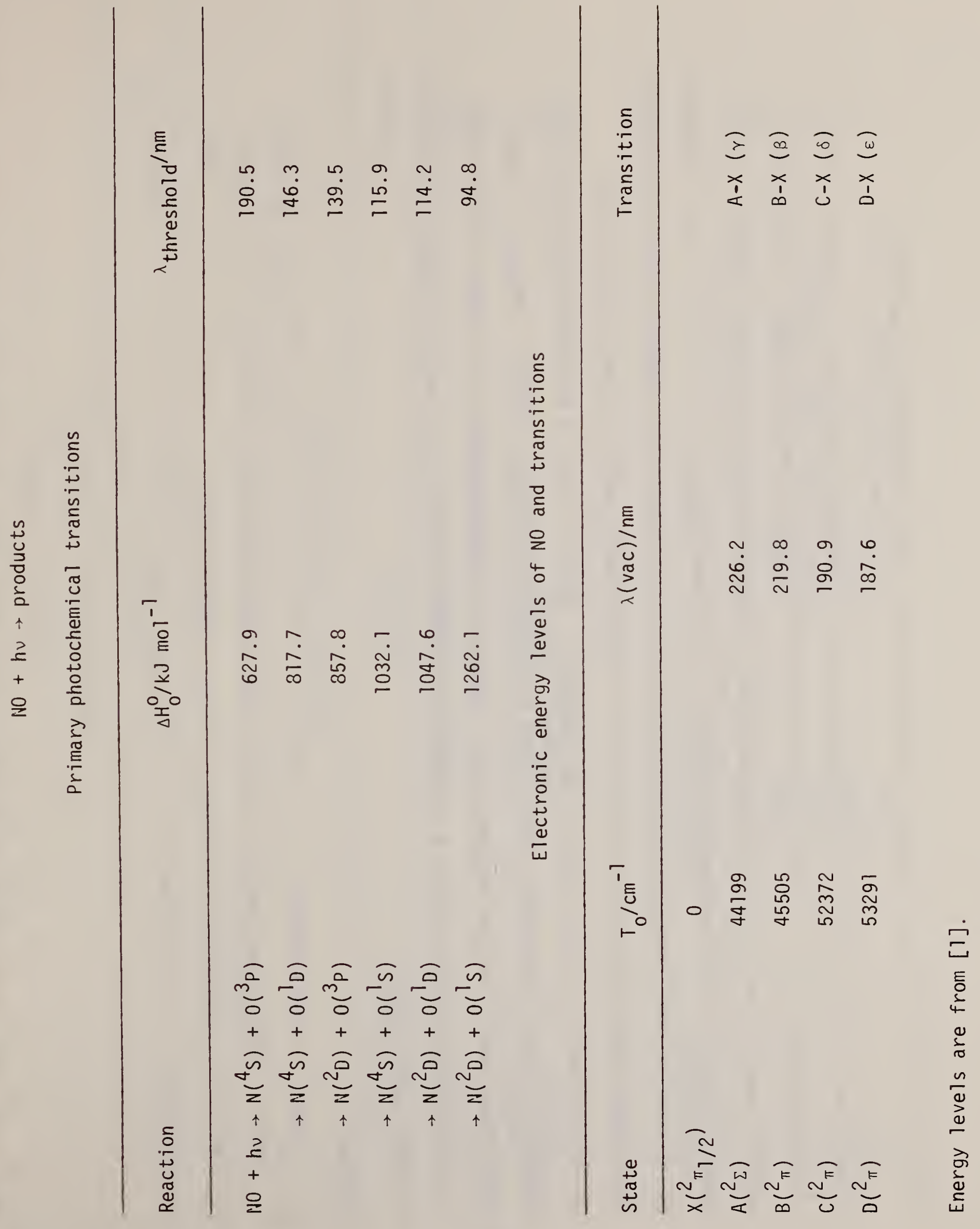




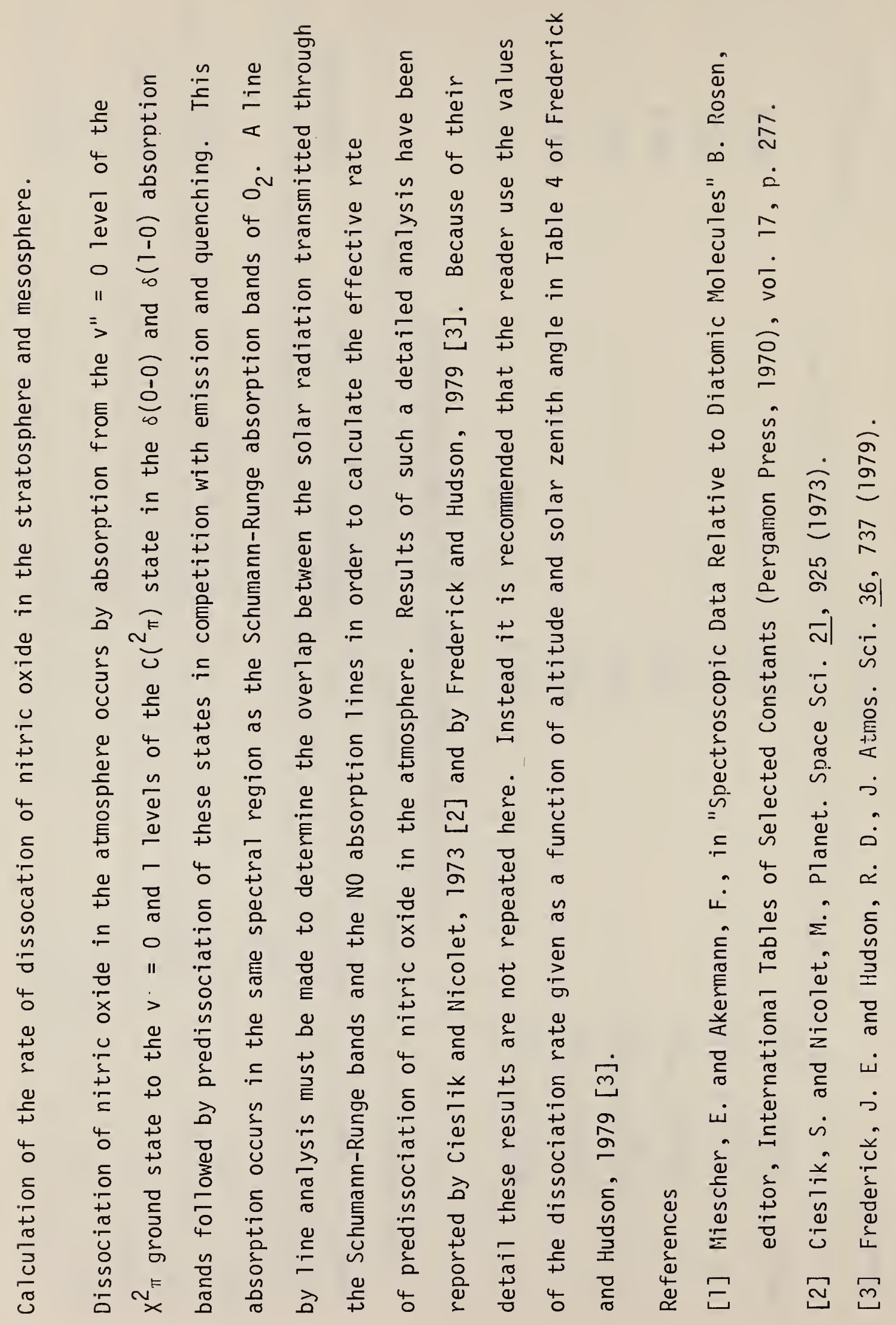




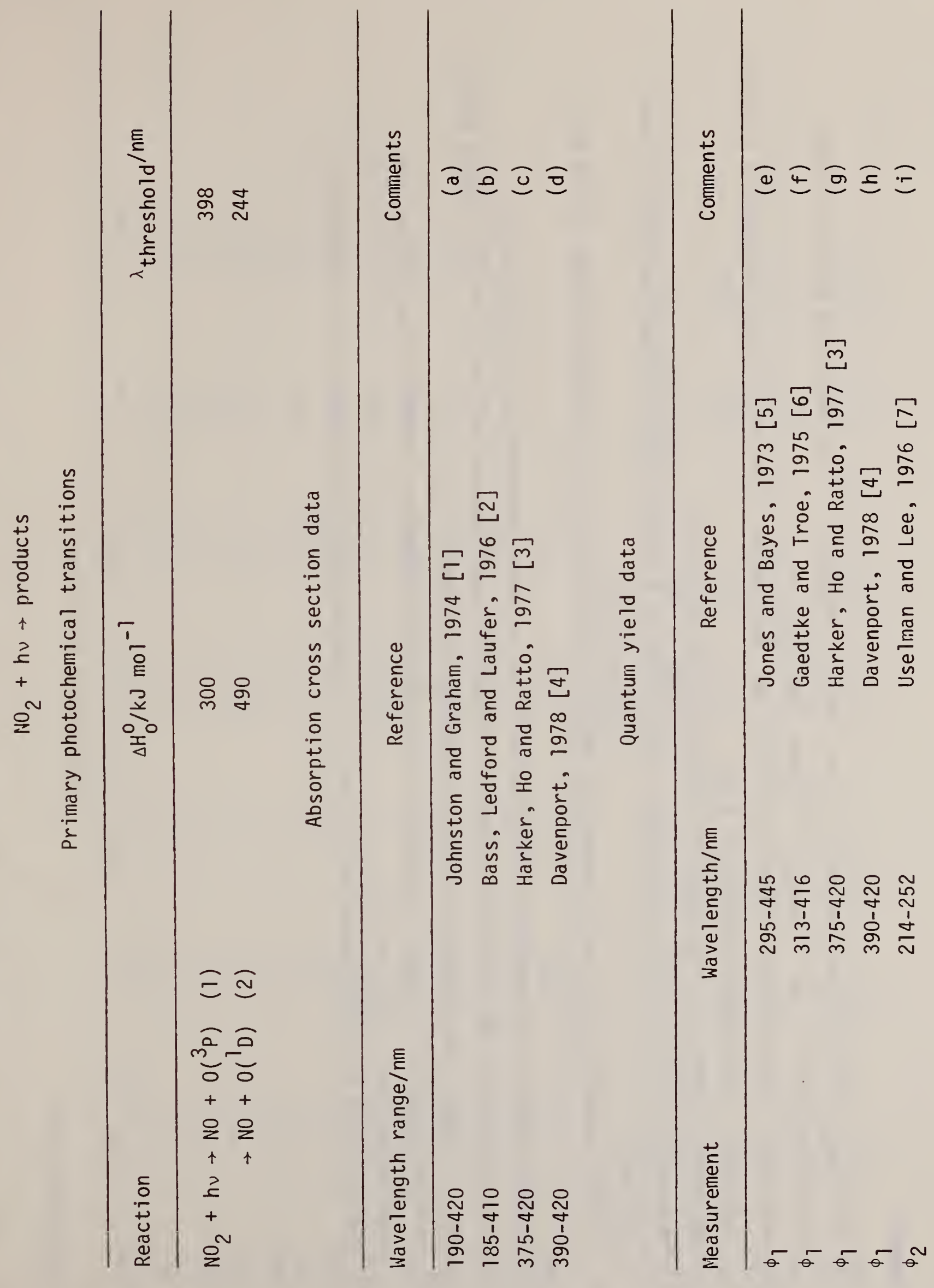




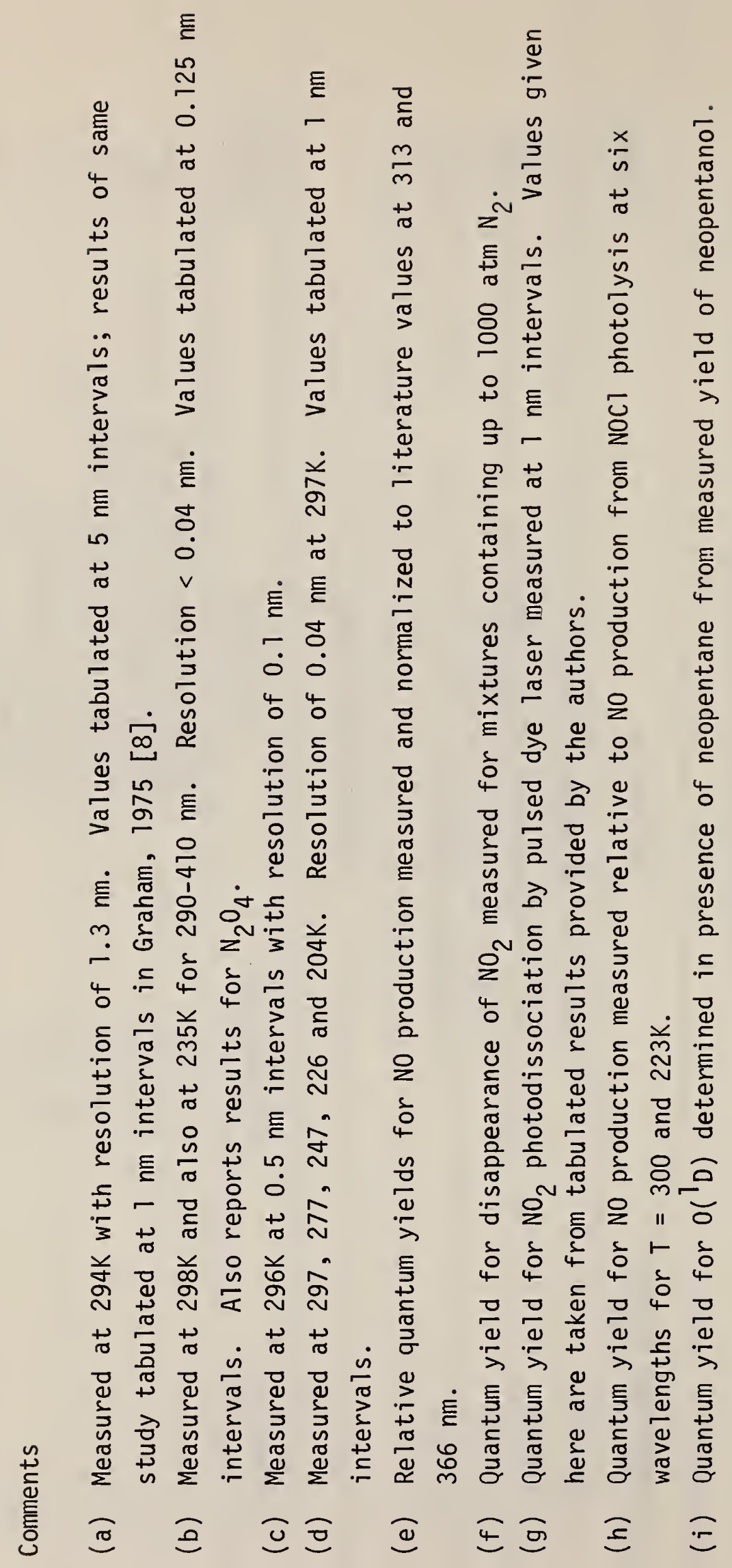




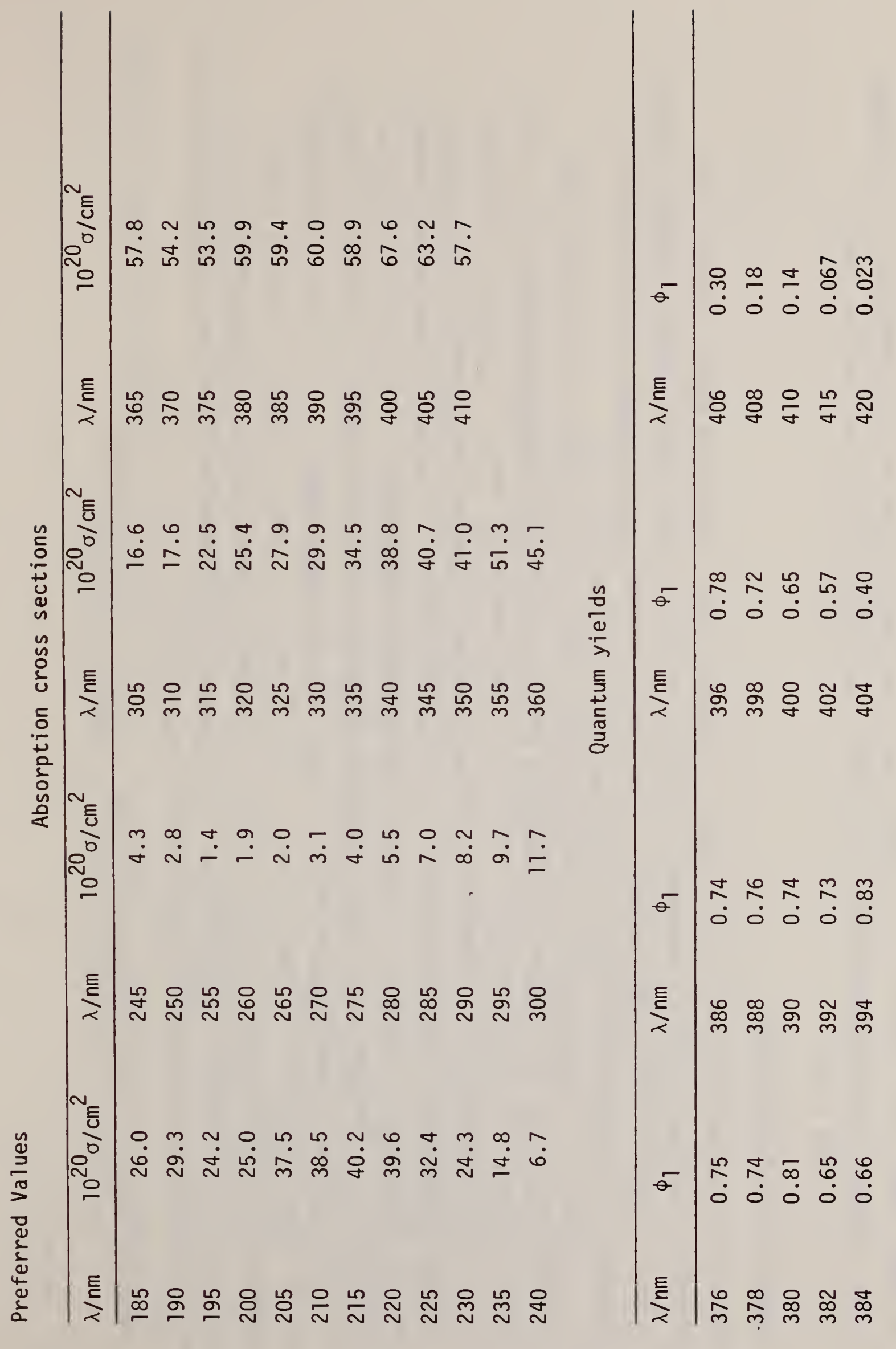




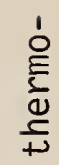

$\frac{\mathscr{c}}{+2}$

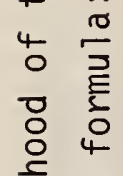

宅

함

๑

$\frac{\mathscr{E}}{\Phi}$

$\therefore \quad \stackrel{0}{0}$

थ

ב

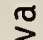

$>\frac{n}{+}$

둥

Ф)

¿

3

¿ั ષิ

․․ㅇํㅇ

\section{ఫิ}

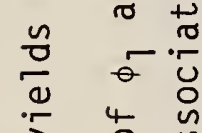

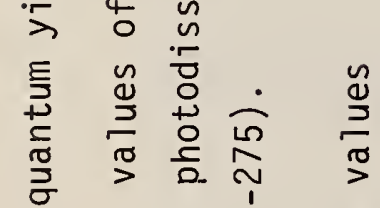

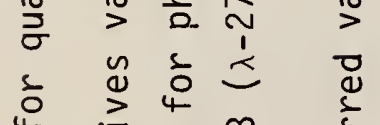

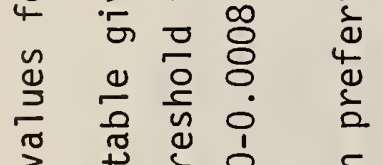

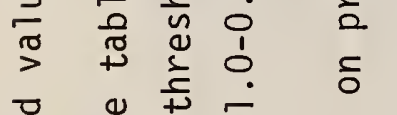

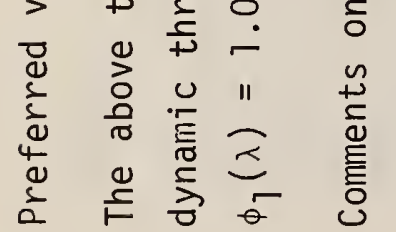

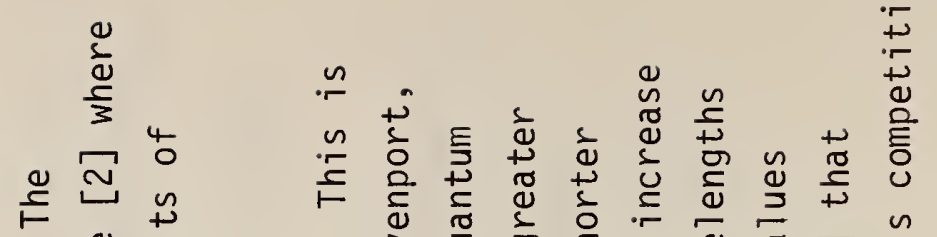

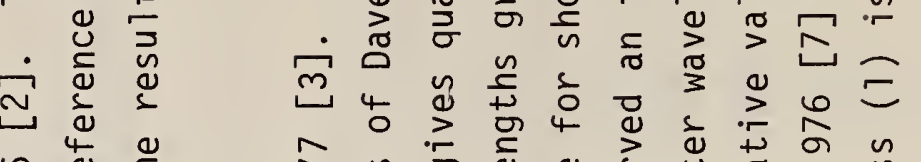

0 \&

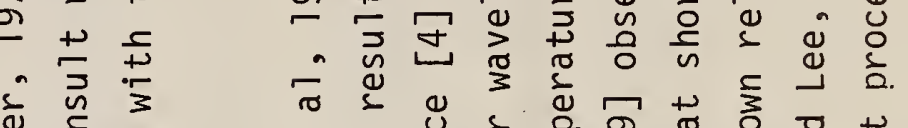

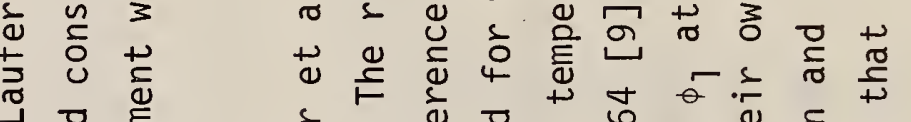

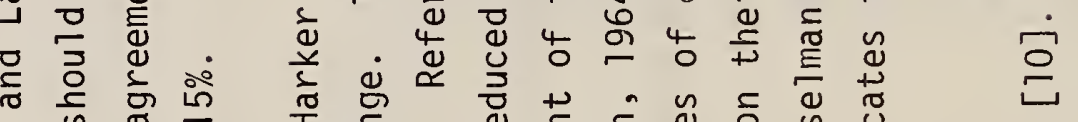

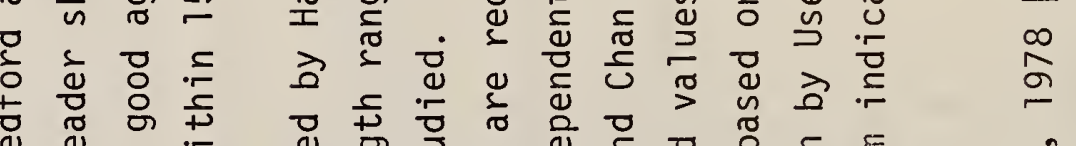

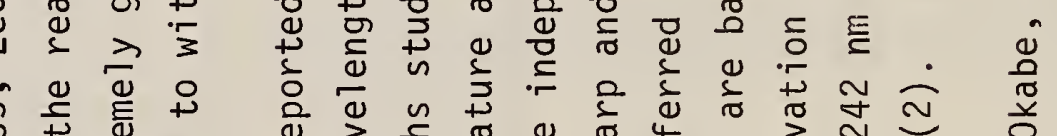

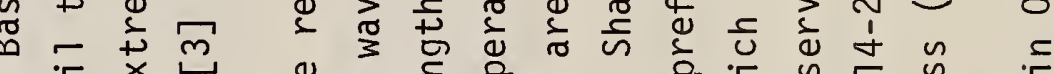
崩 $\cong$ व

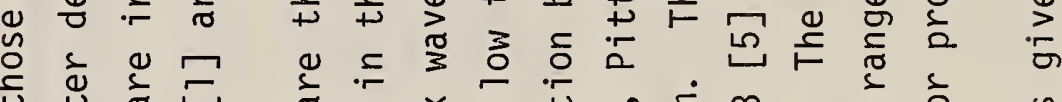

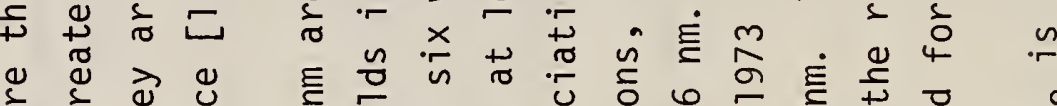

先 $\delta$.

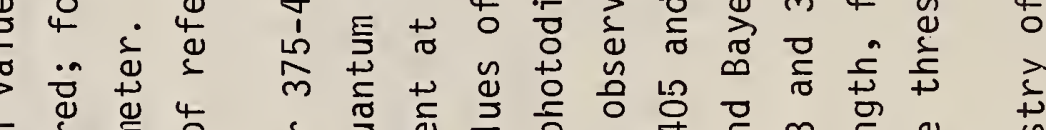

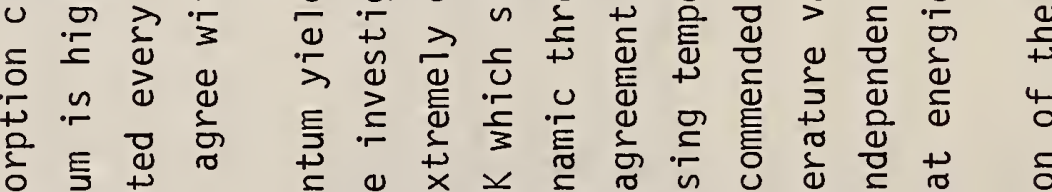

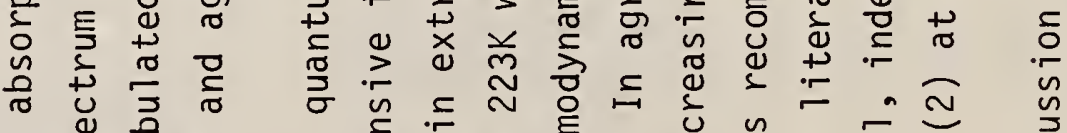

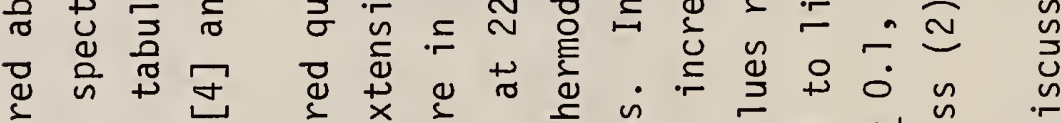

¿

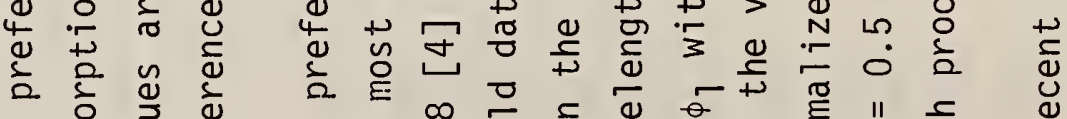

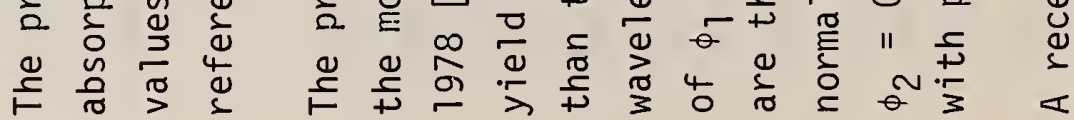




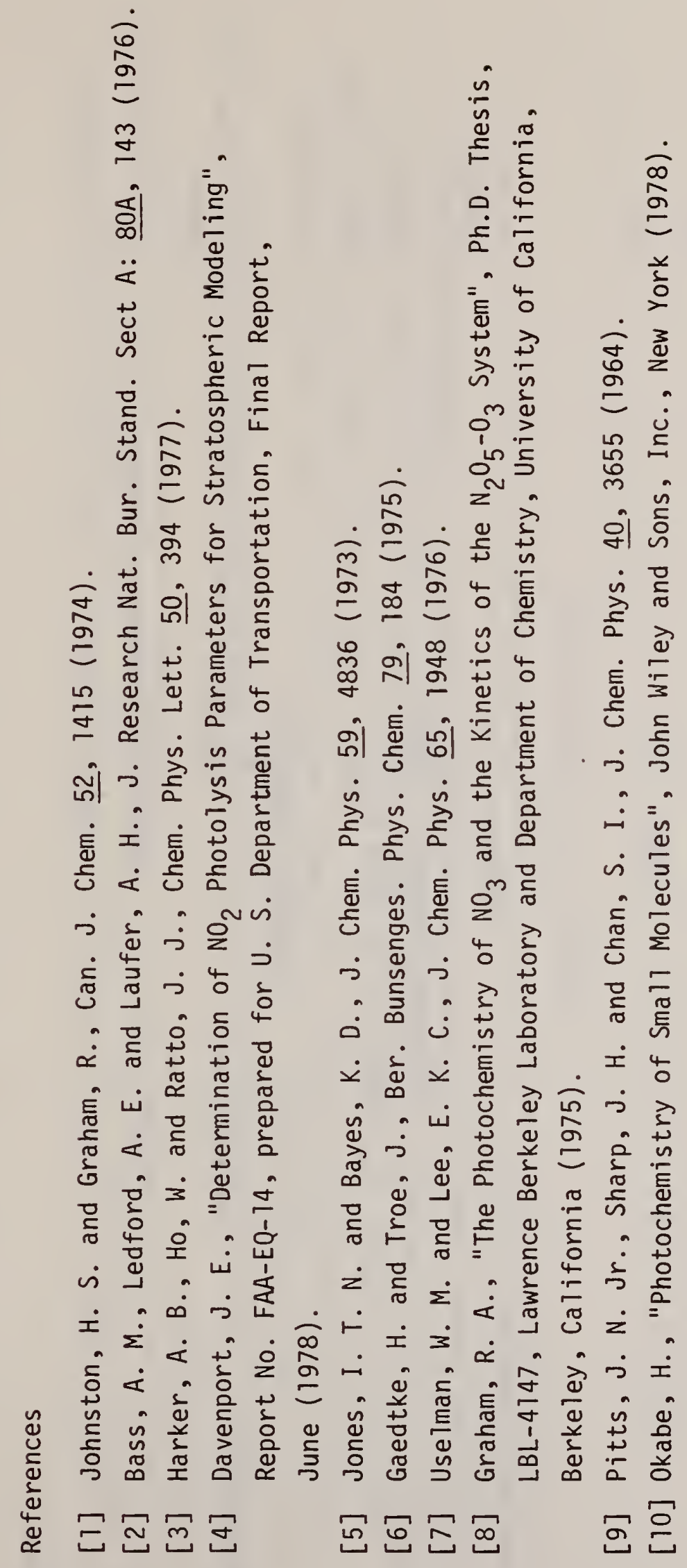




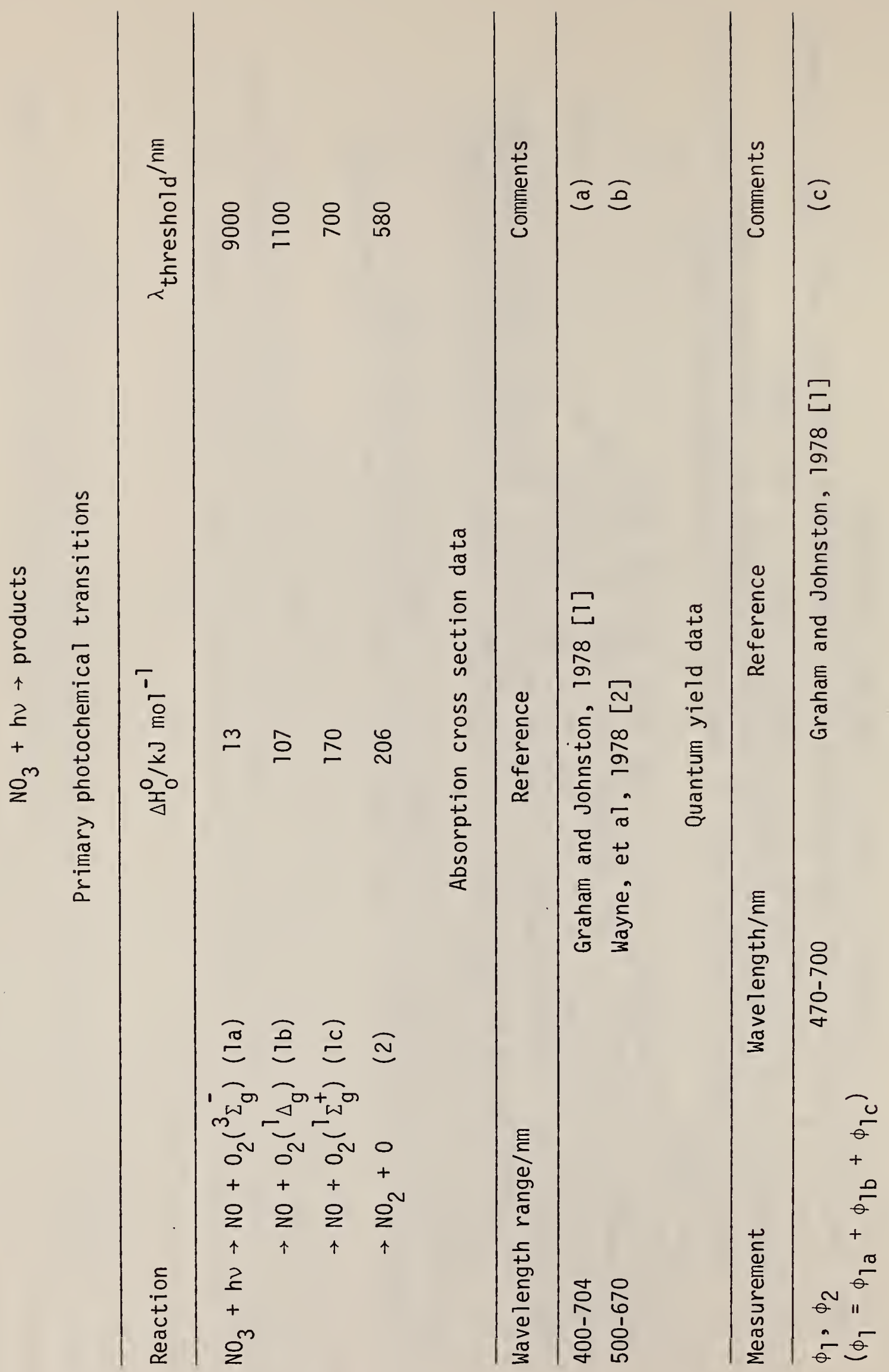




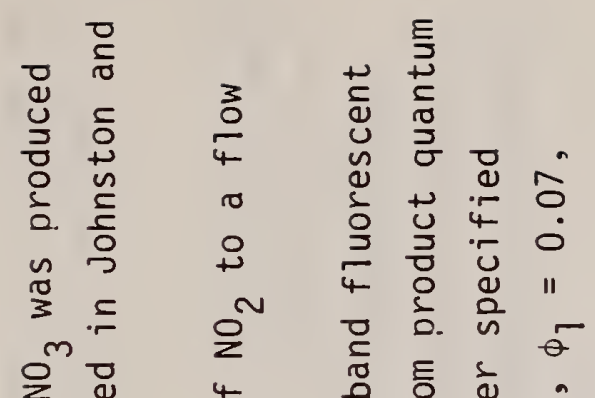

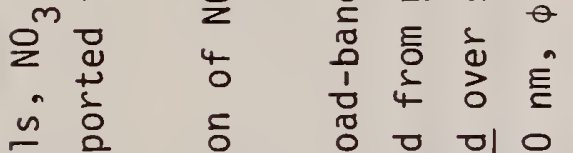

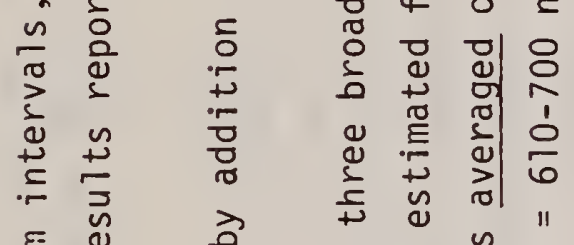

$$
\begin{aligned}
& \text { घ }
\end{aligned}
$$

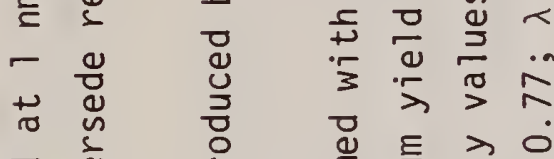

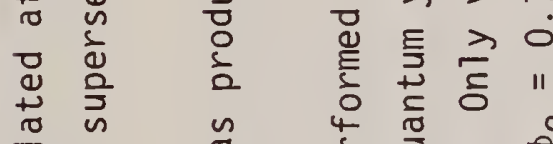

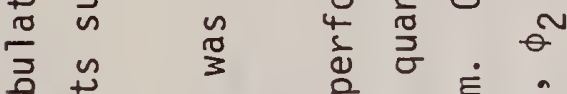

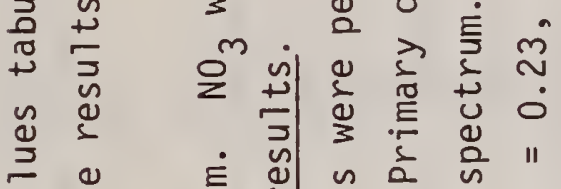

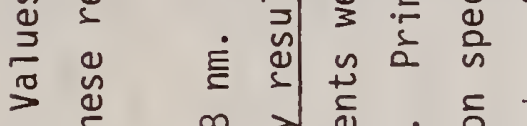

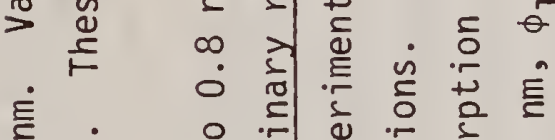

$$
\begin{aligned}
& \text { m }
\end{aligned}
$$

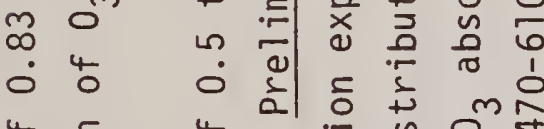

$$
\begin{aligned}
& \text { 4 ᄃ } \\
& \text { ᄃㄷำ }
\end{aligned}
$$

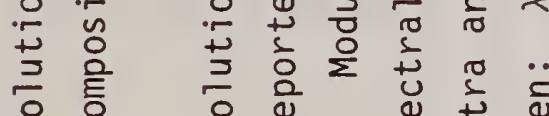

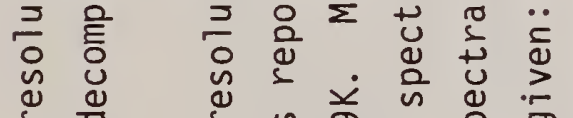

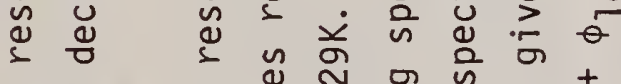

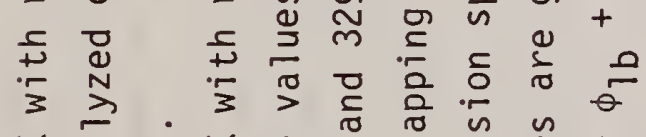

$$
\begin{aligned}
& \text { 并 } \\
& \text { กิ }
\end{aligned}
$$

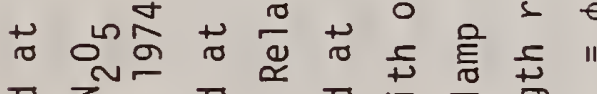

$$
\begin{aligned}
& \text { ¿ }
\end{aligned}
$$

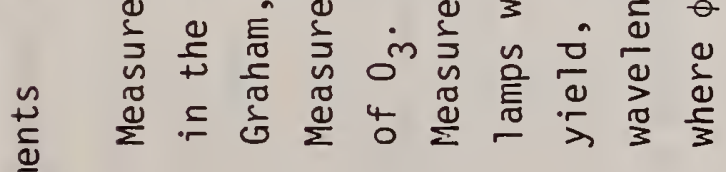

$$
\begin{aligned}
& \text { है ฮ } 0 \text { ป }
\end{aligned}
$$




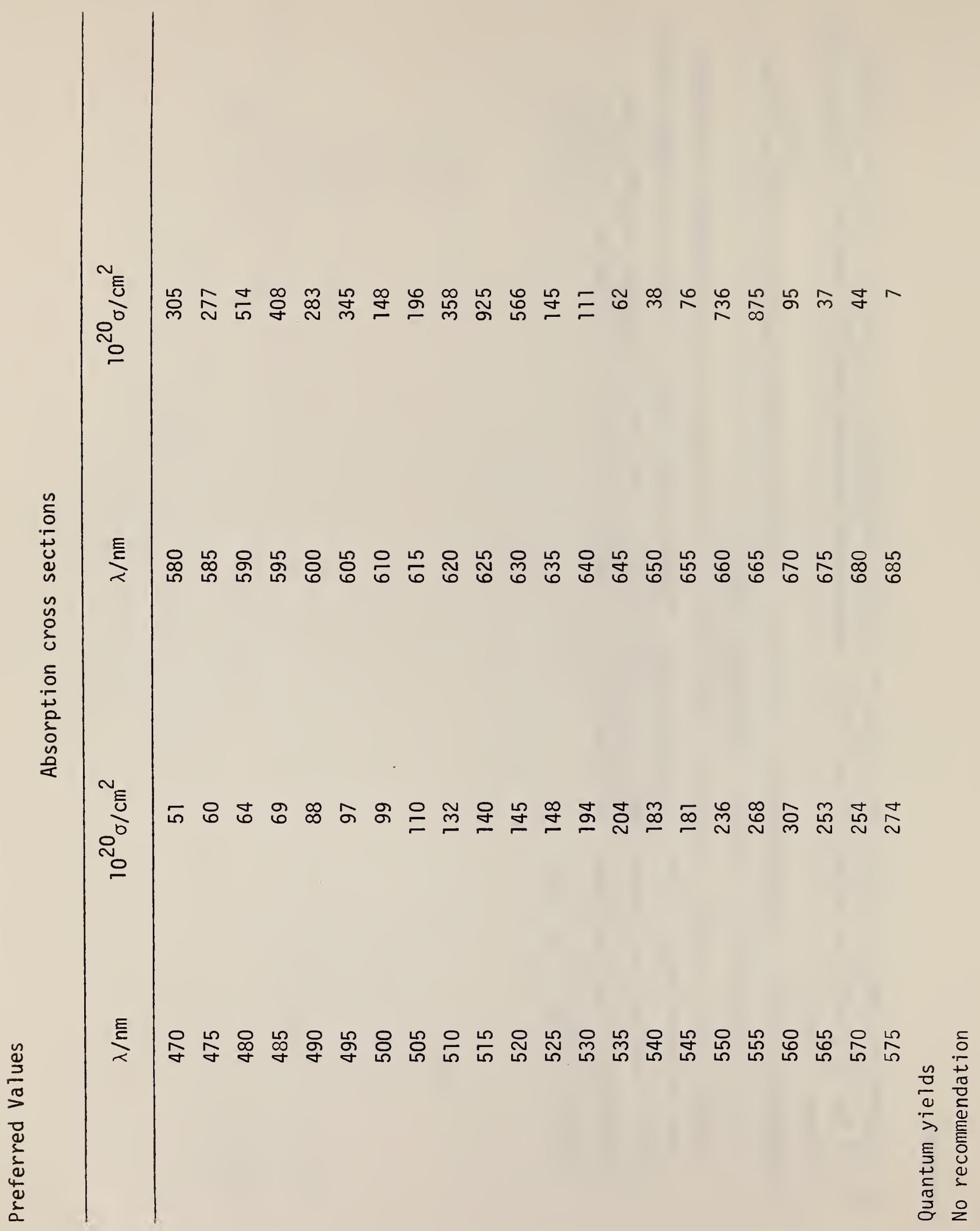




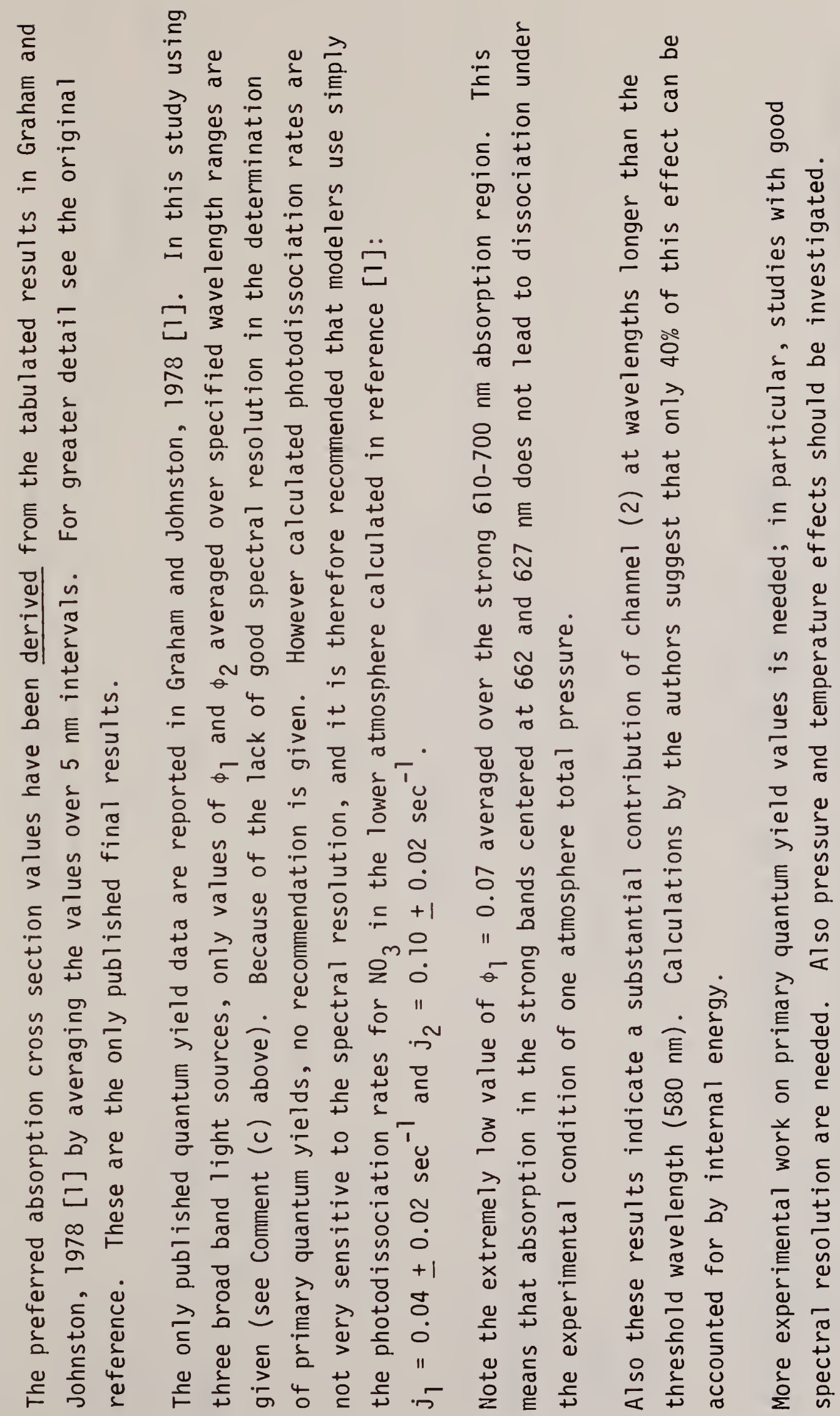




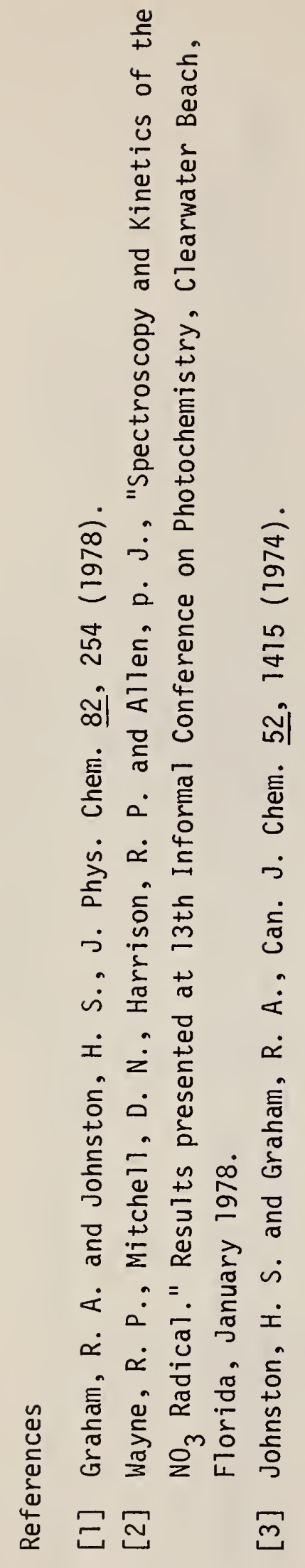




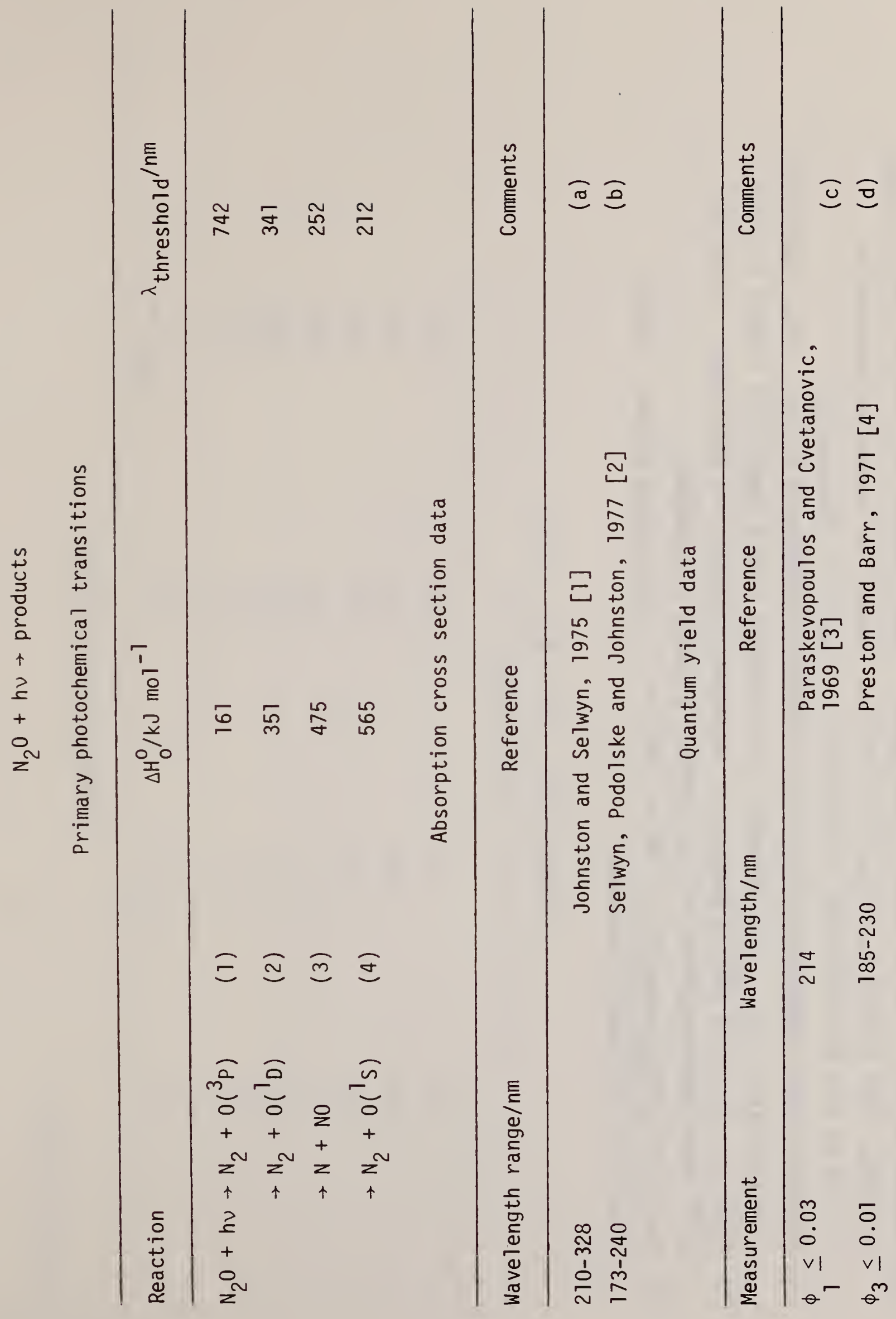




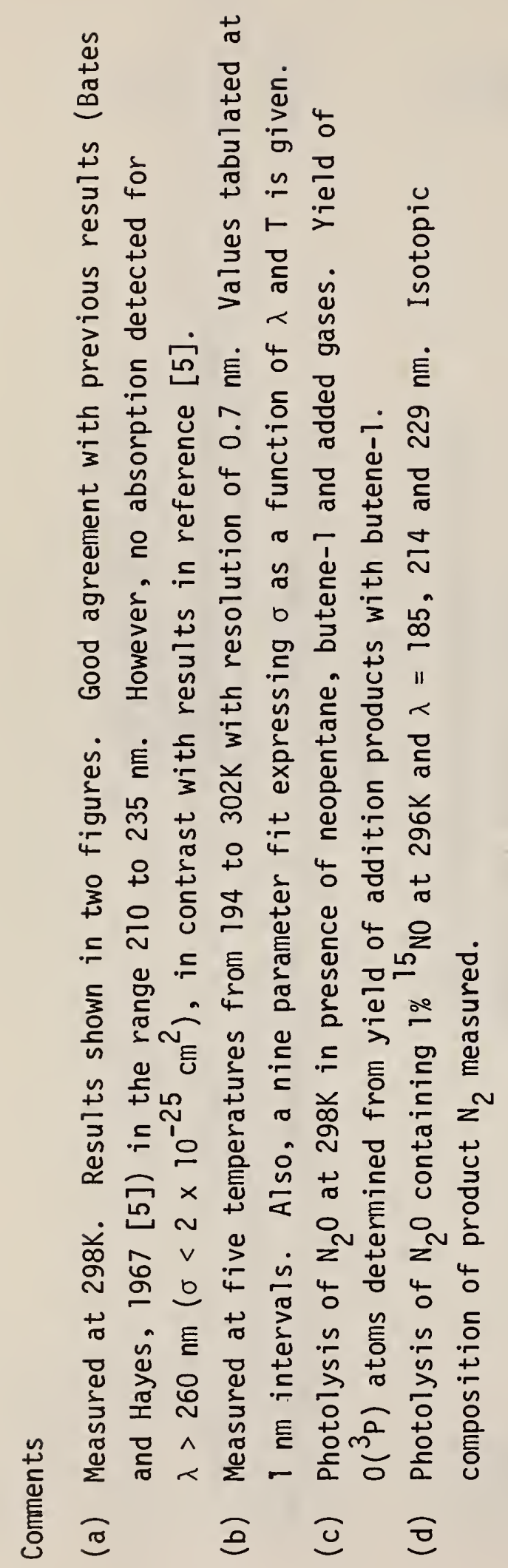




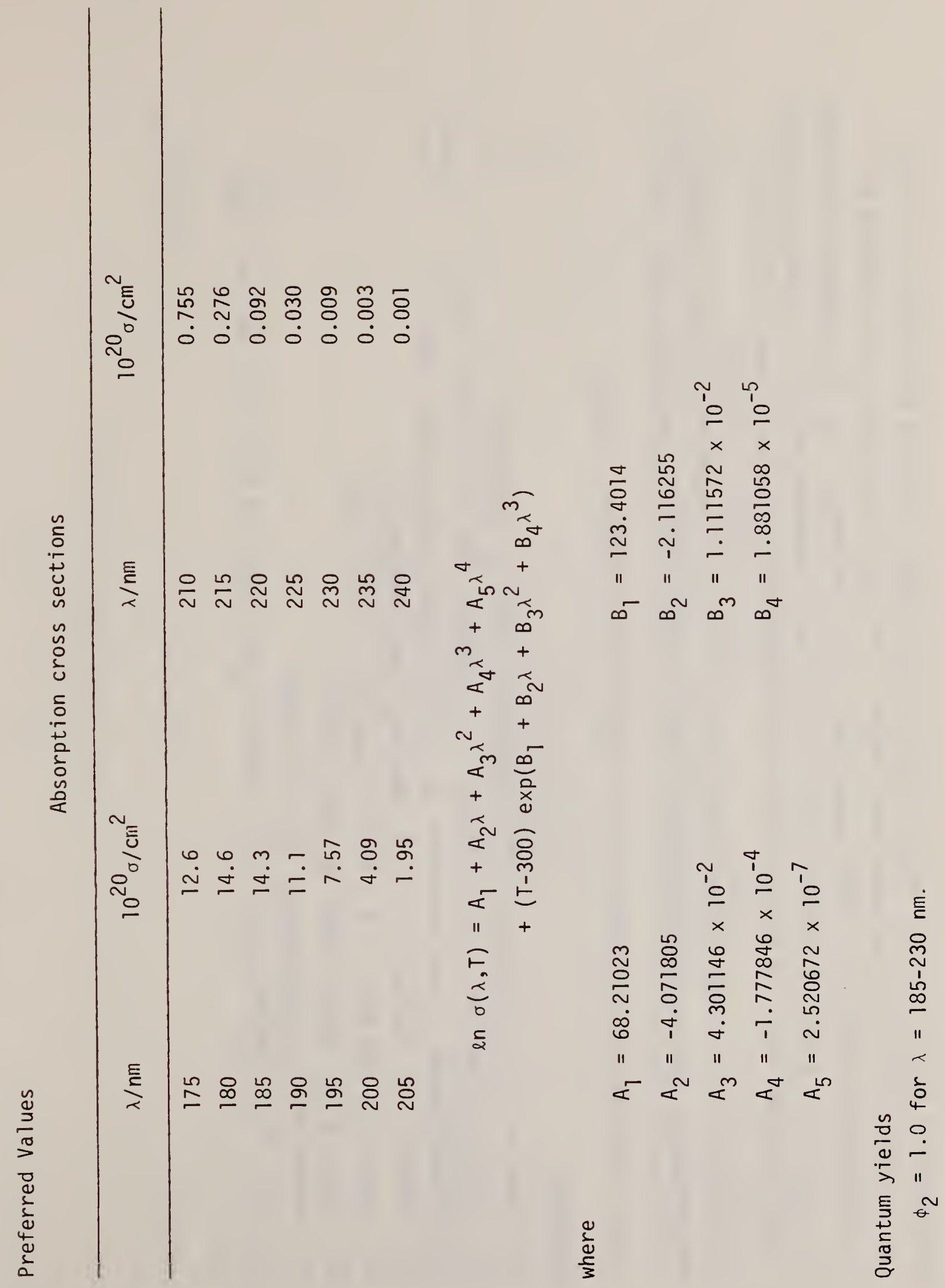




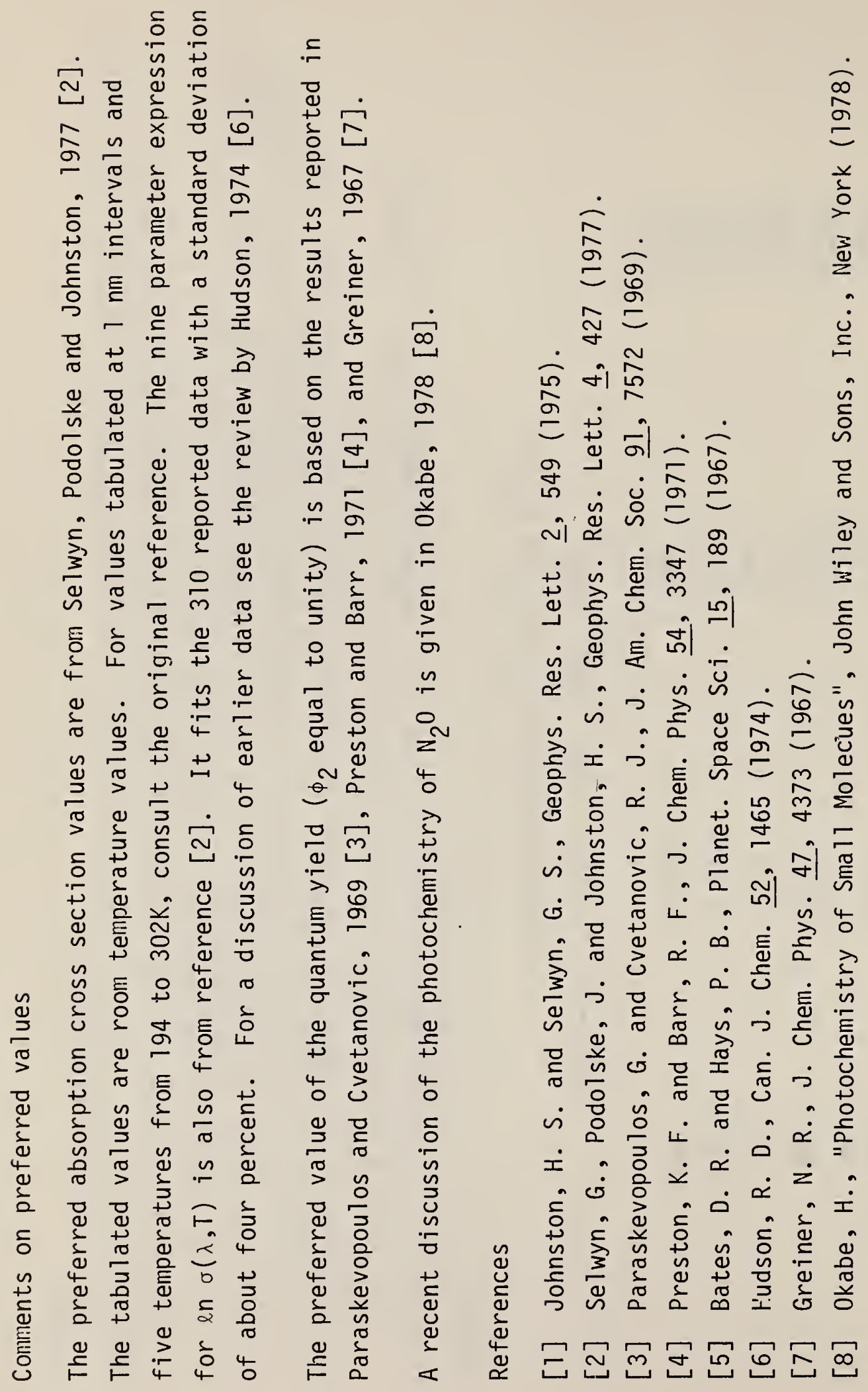




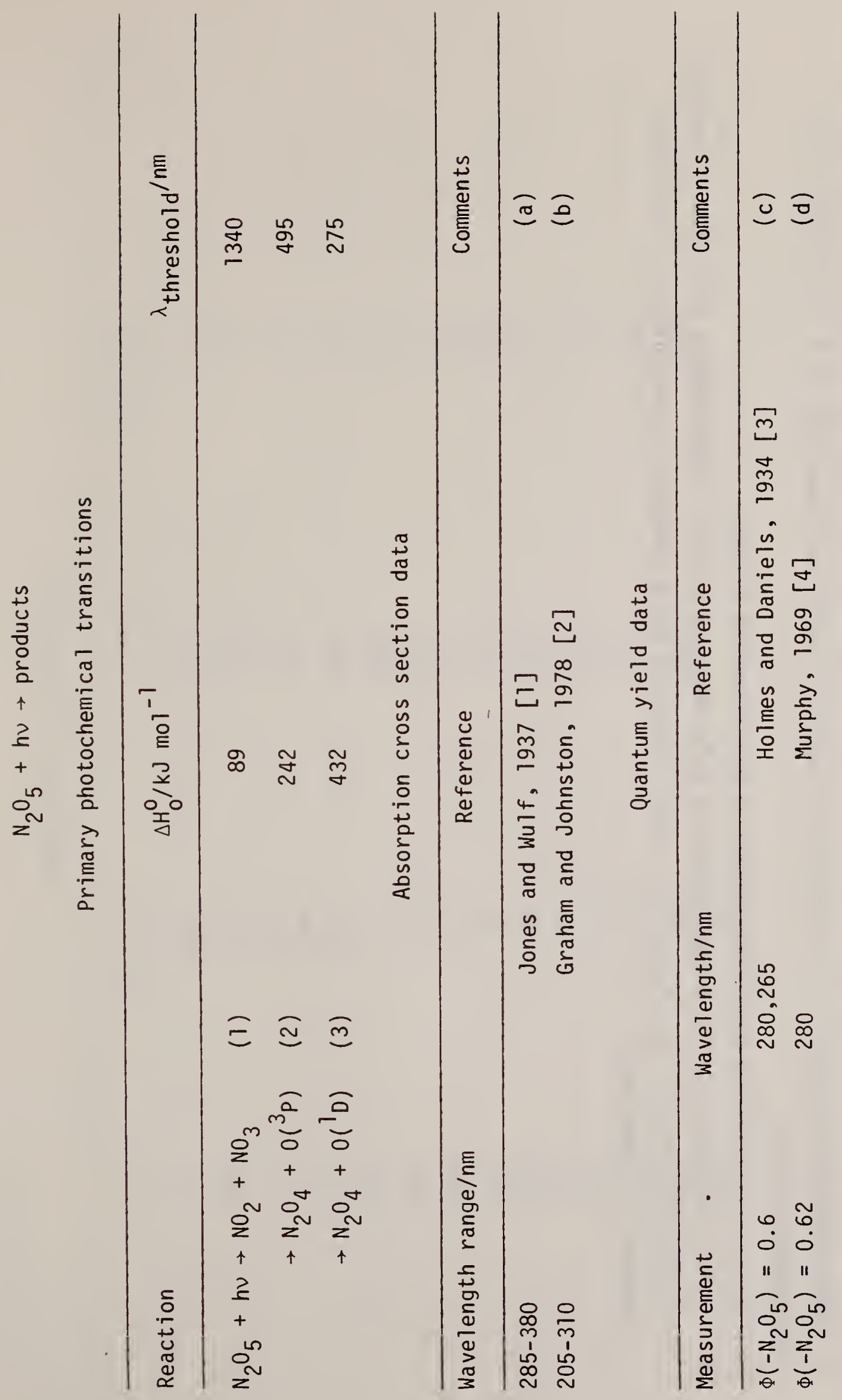




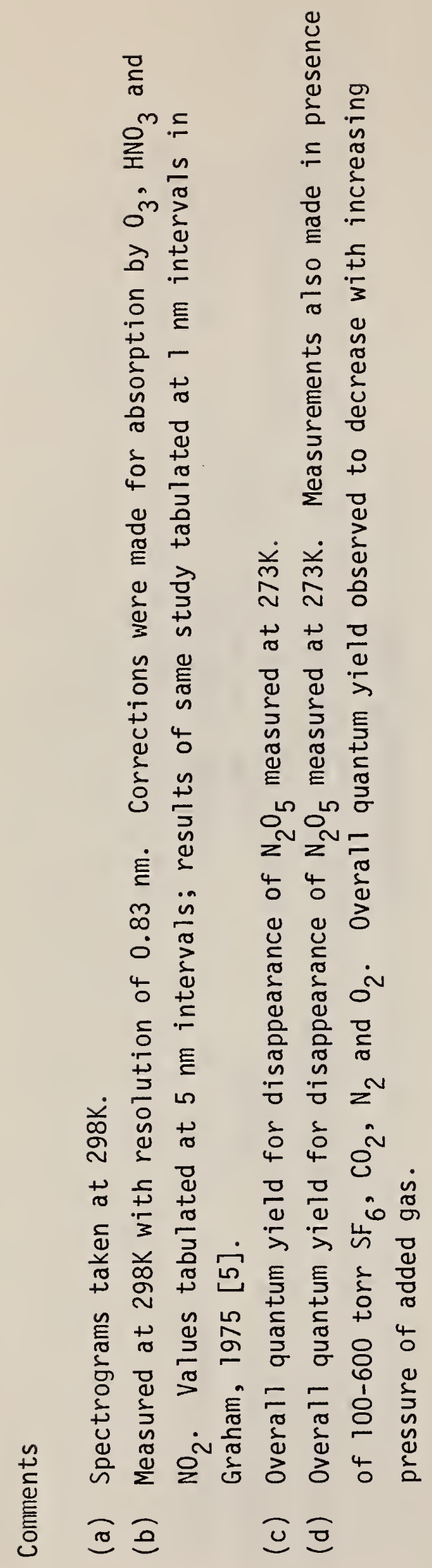




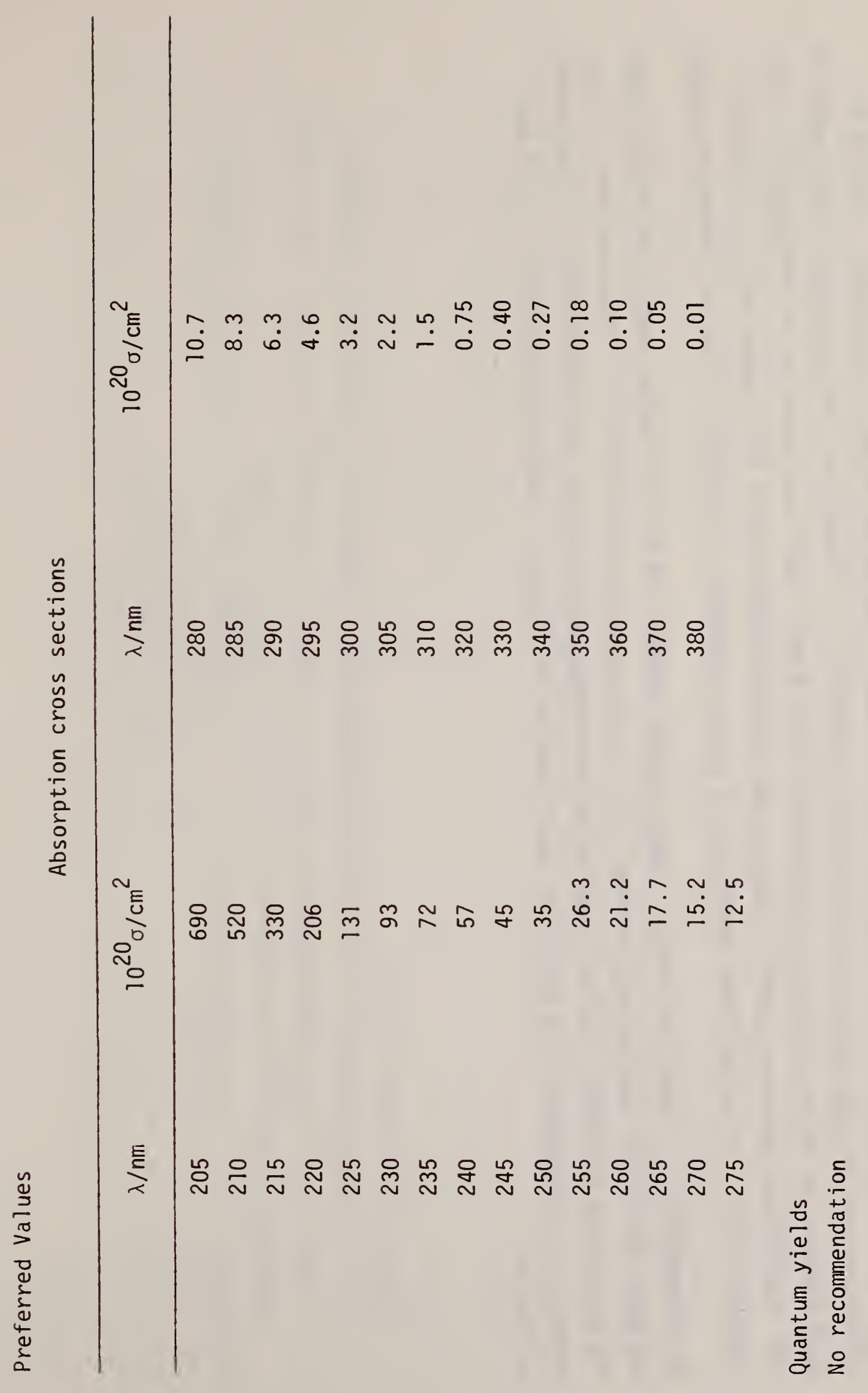




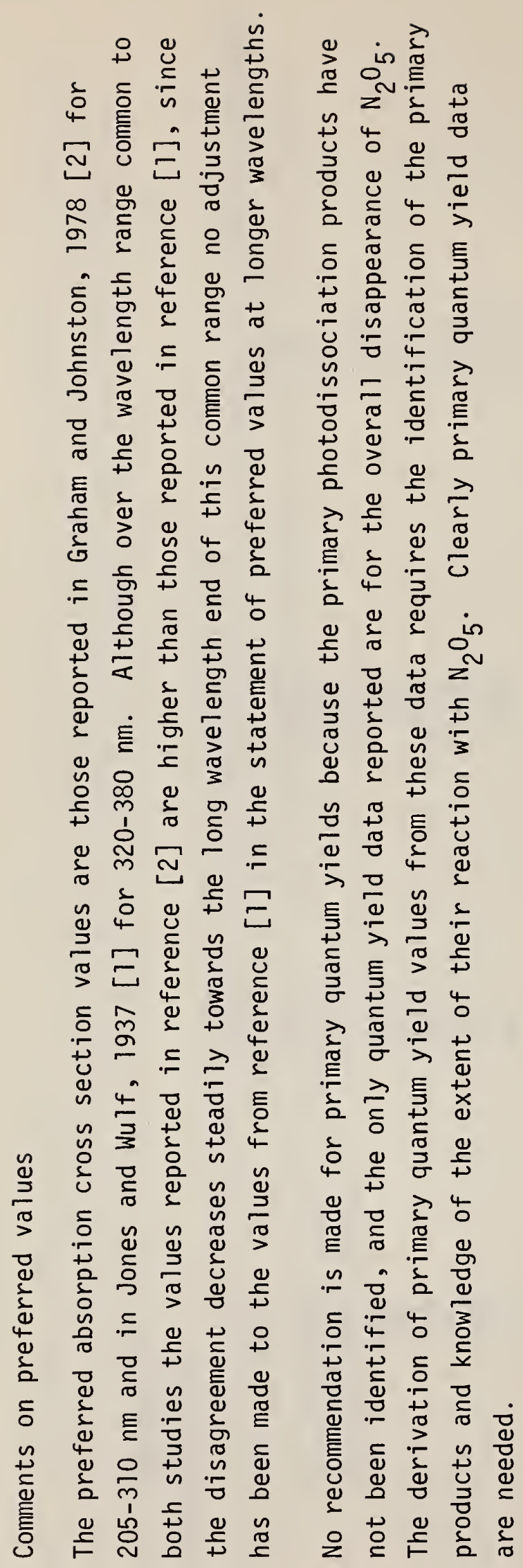




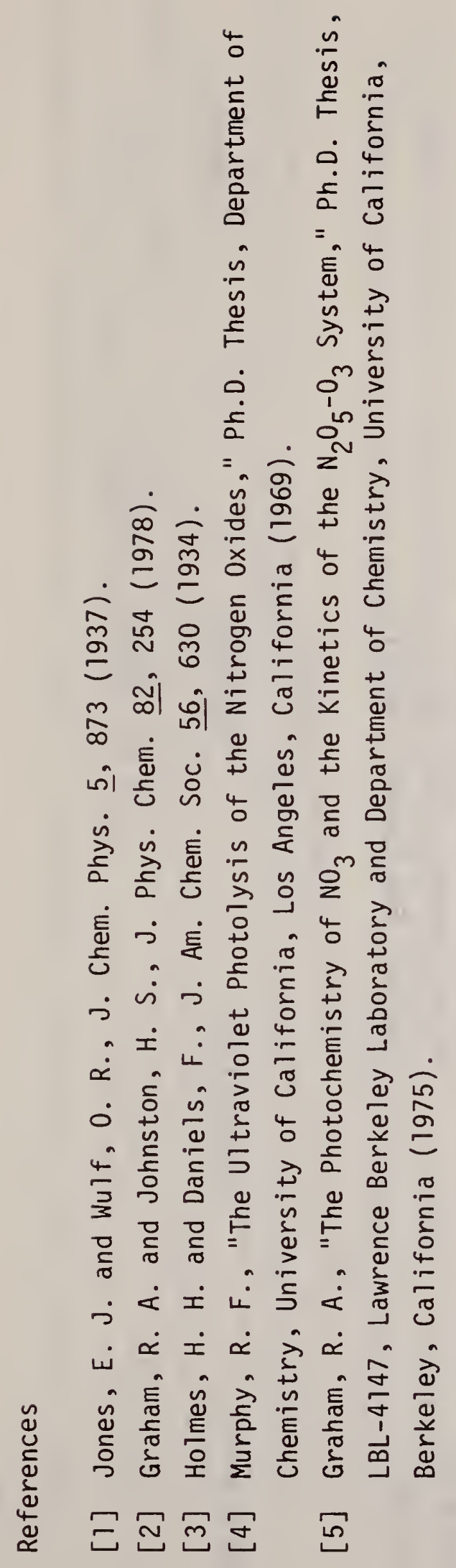




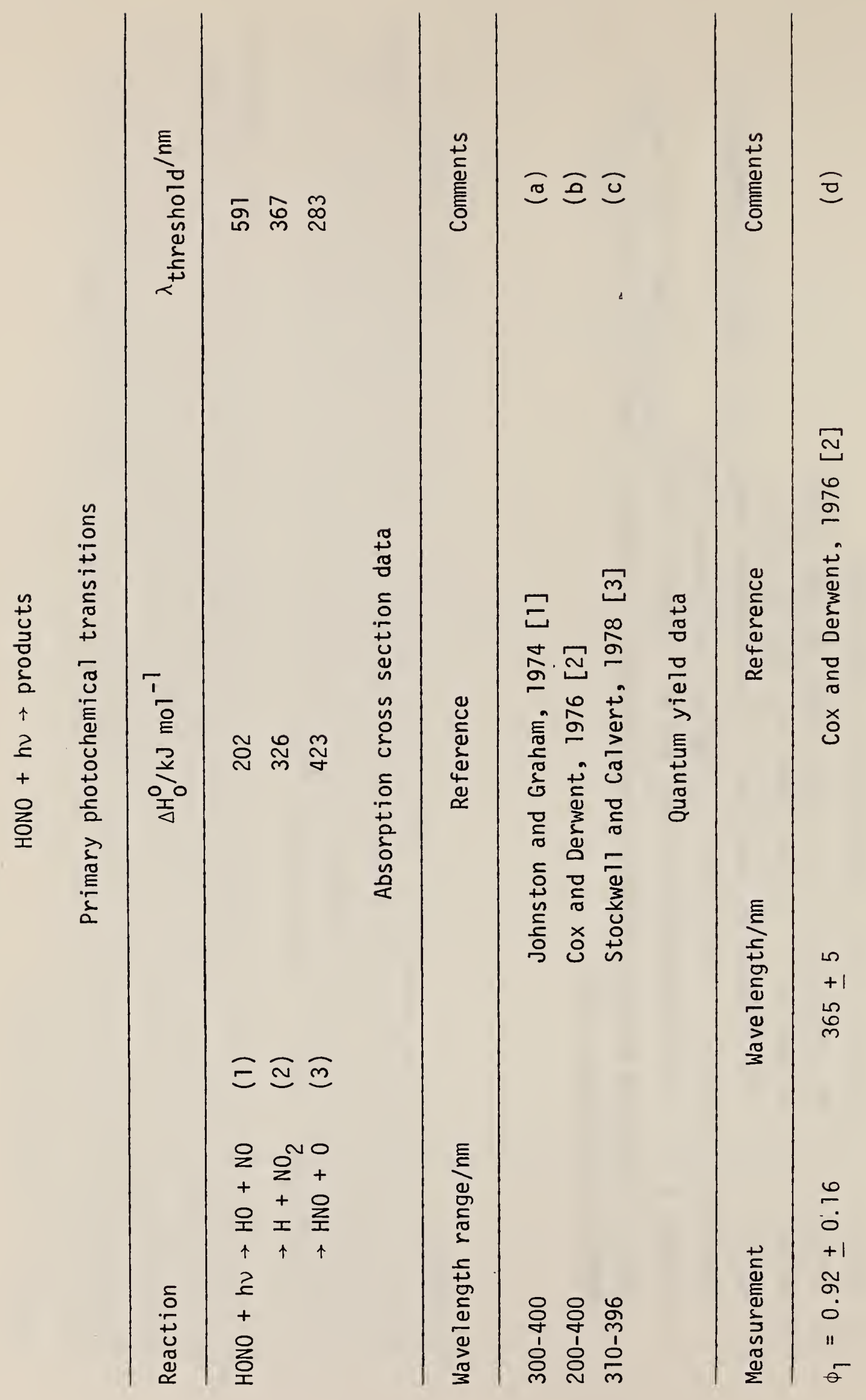




$$
\begin{aligned}
& \text { 产喜高 }
\end{aligned}
$$

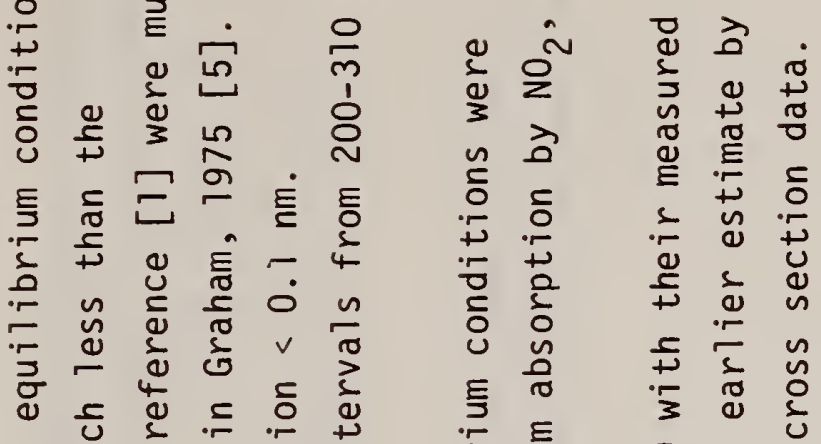

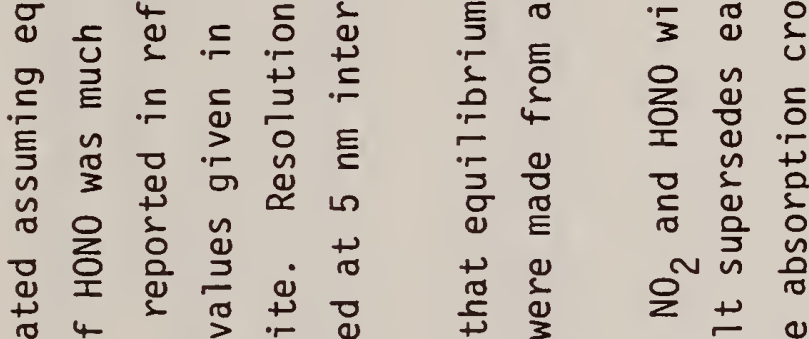

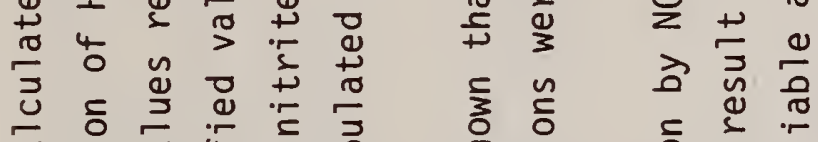

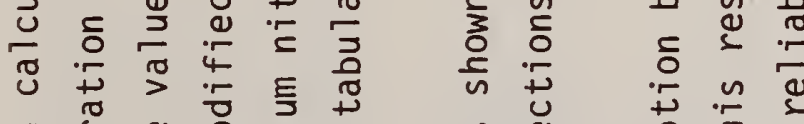

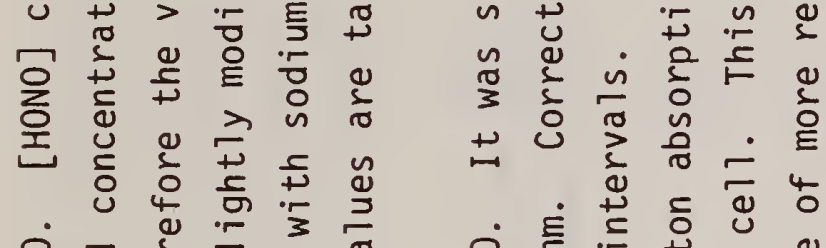

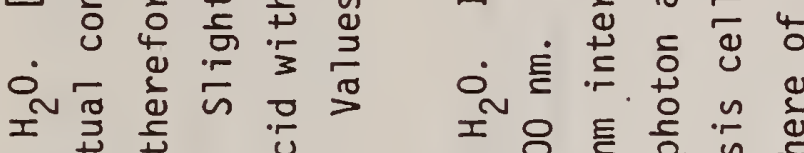

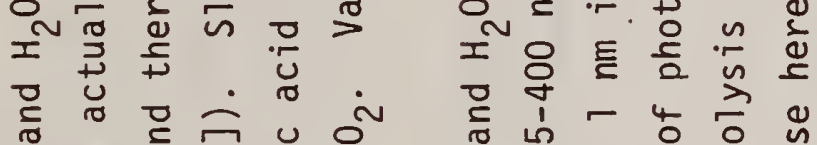

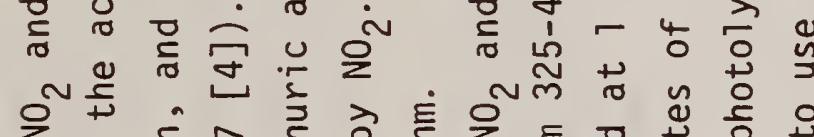

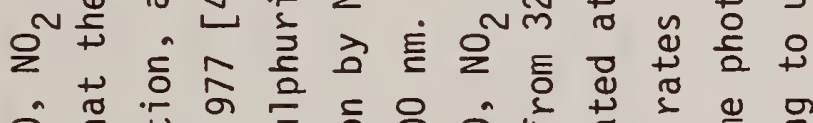

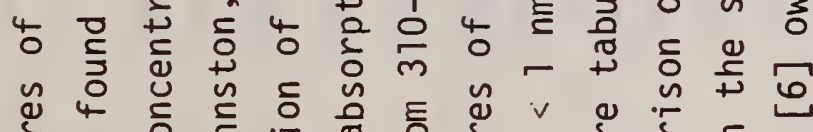

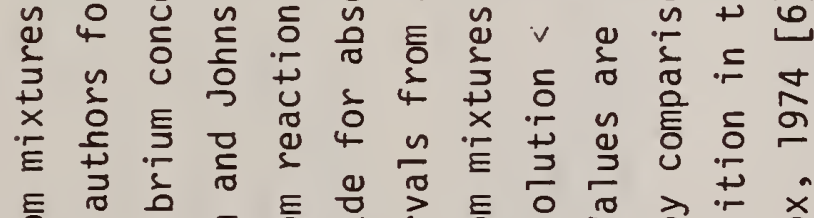

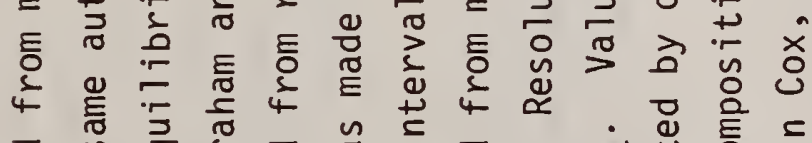

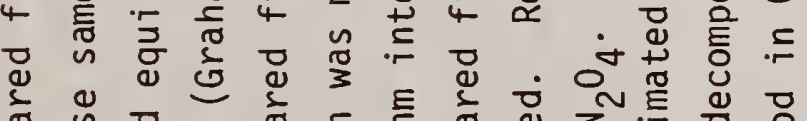

$$
\begin{aligned}
& \text { 売 } \\
& \text { 崩 站 }
\end{aligned}
$$

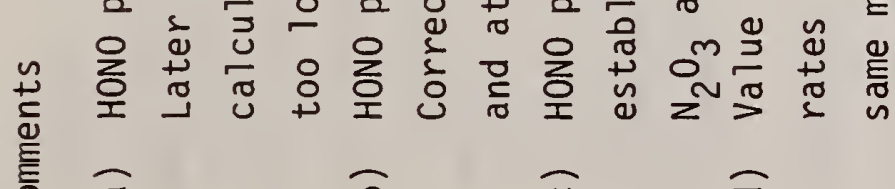

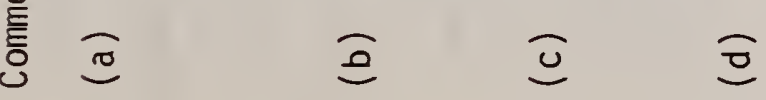




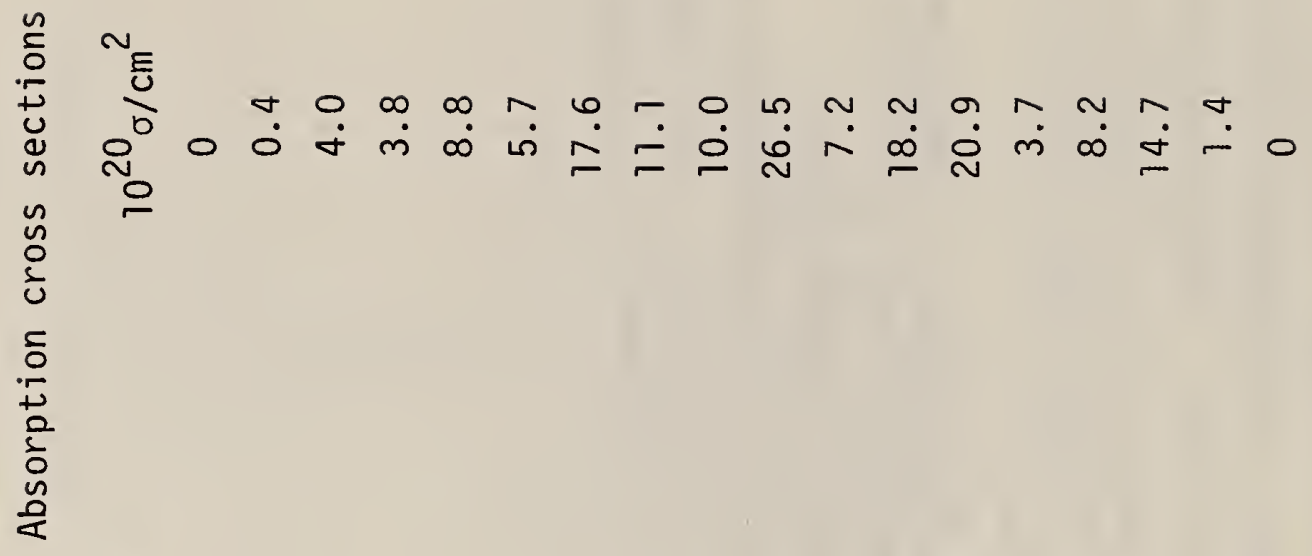

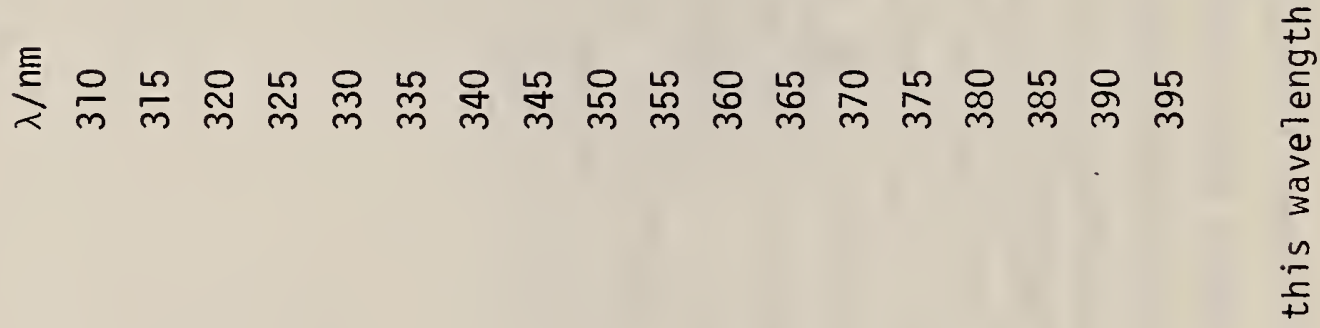

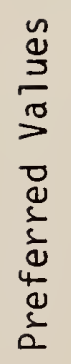




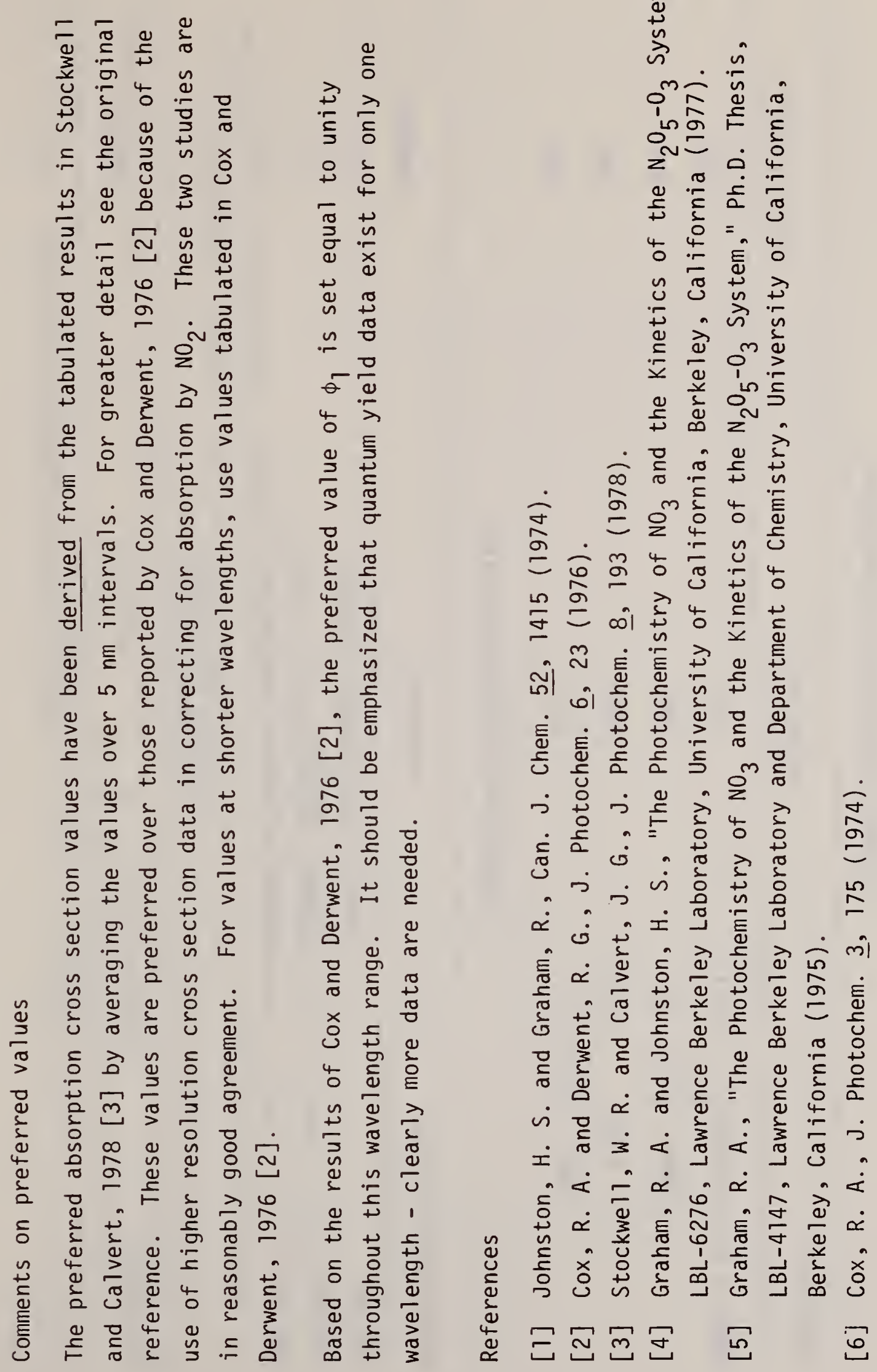




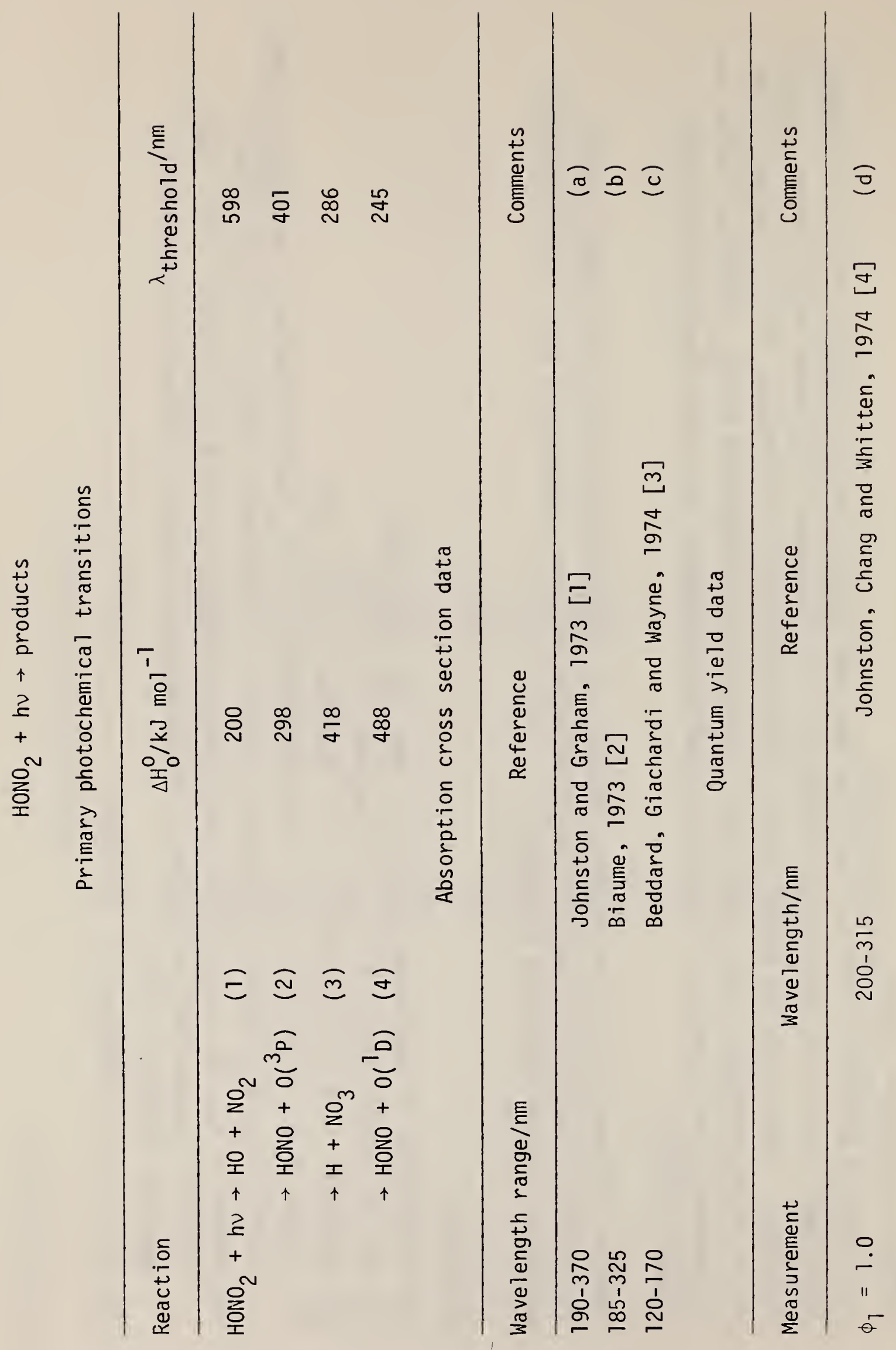




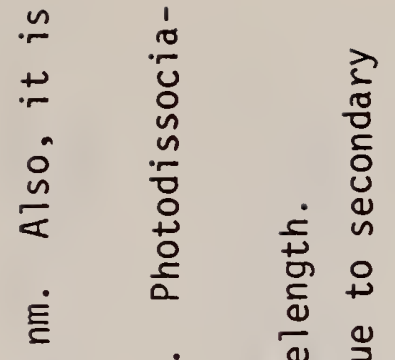

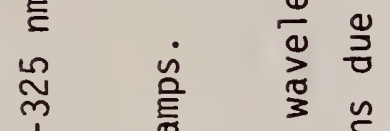

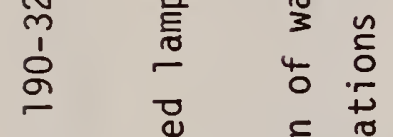

$$
\begin{aligned}
& \text { 焉 }
\end{aligned}
$$

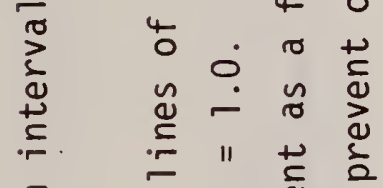

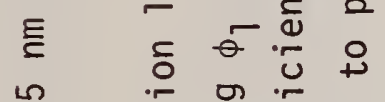

$$
\begin{aligned}
& \text { 品 }
\end{aligned}
$$

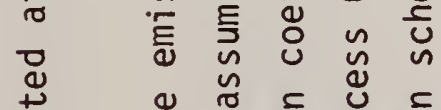

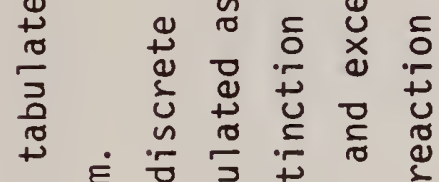

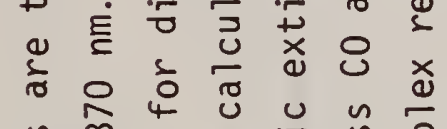

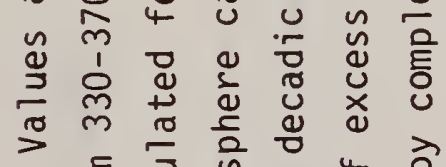

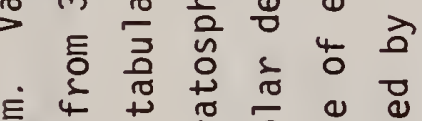

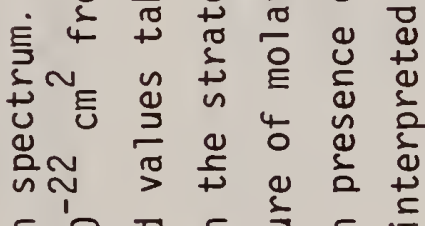

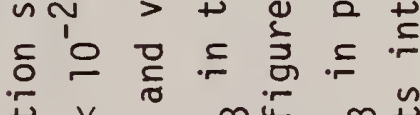

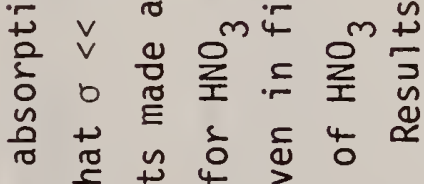

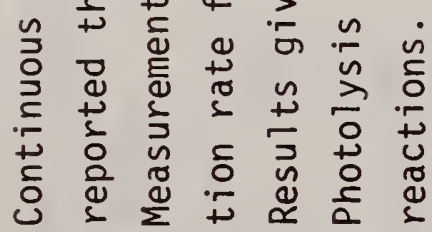

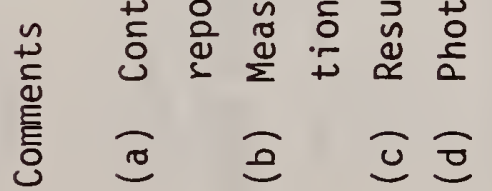




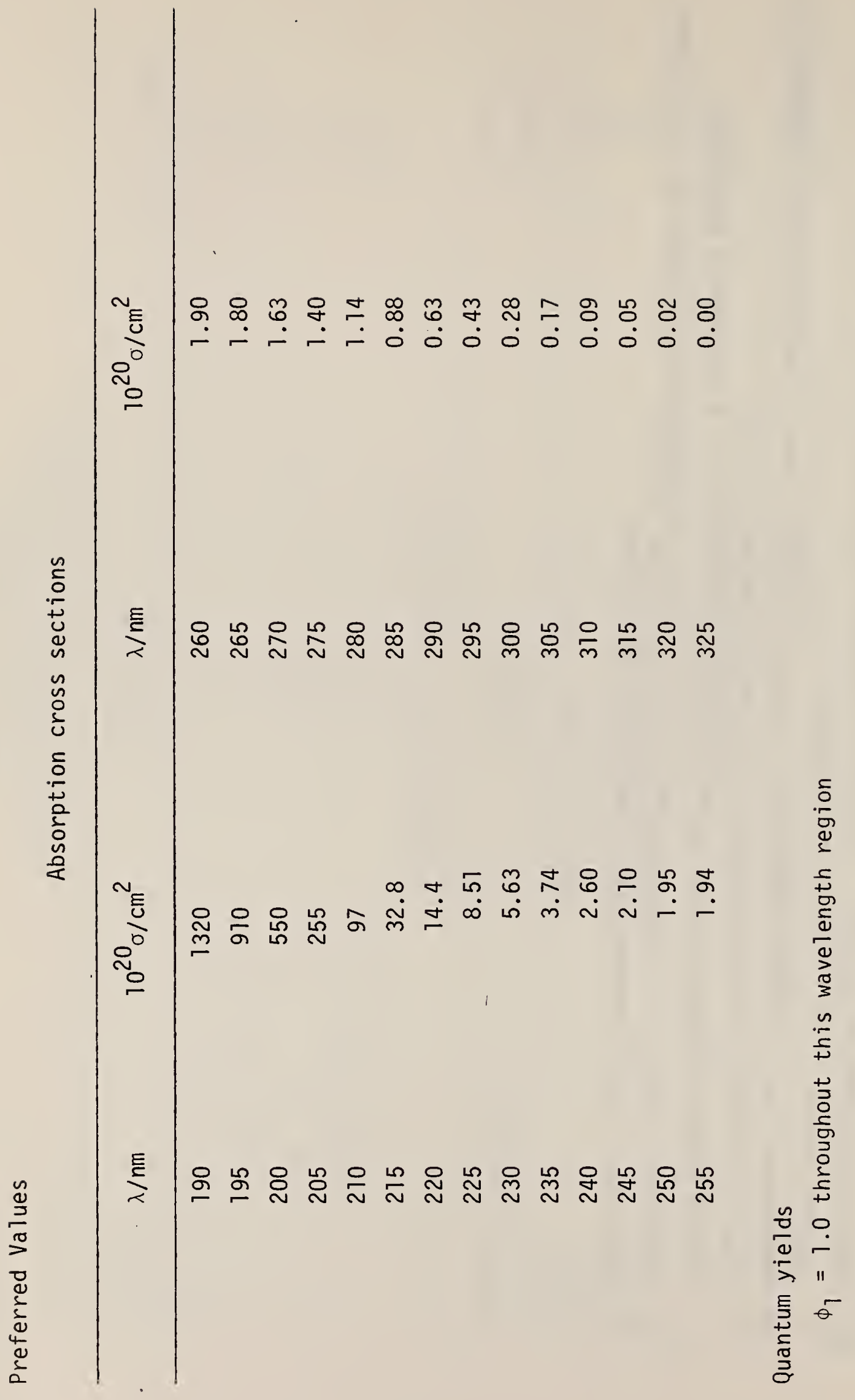




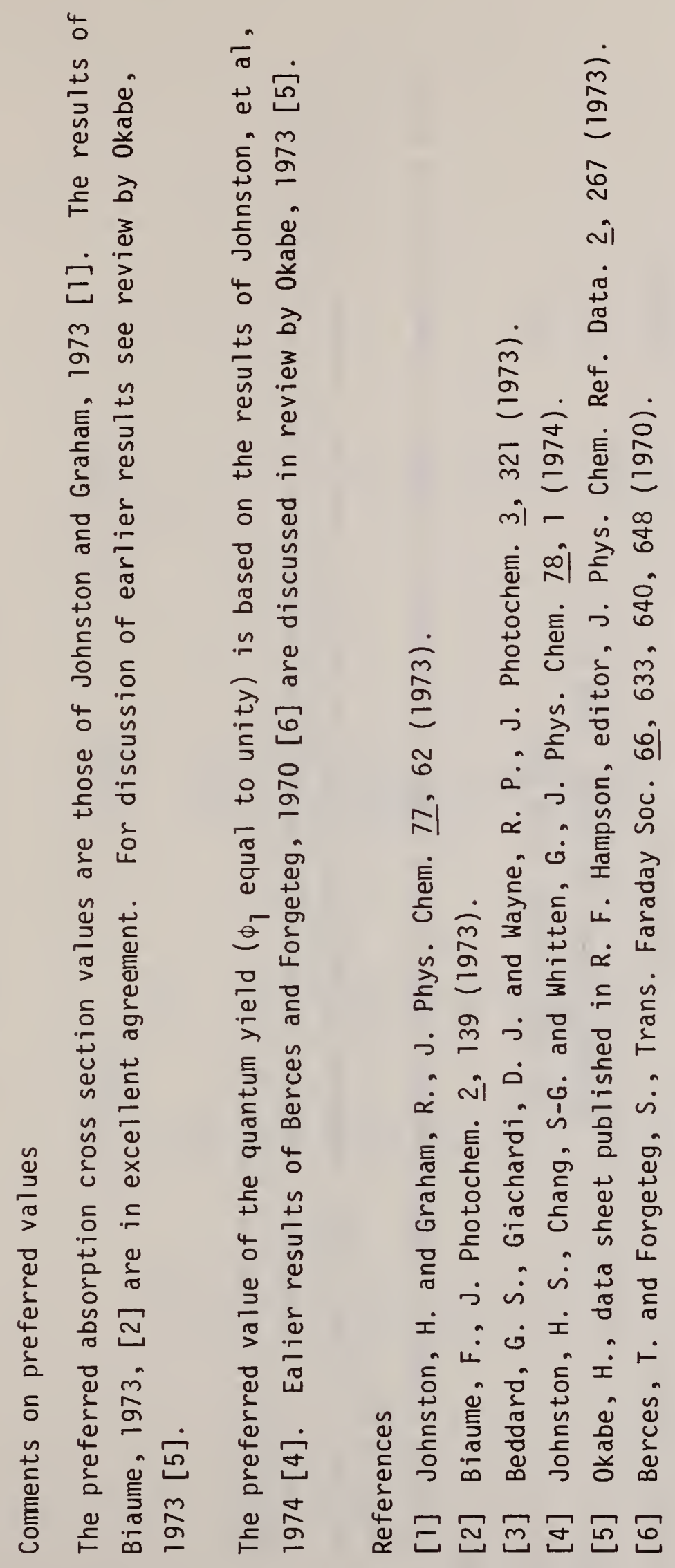




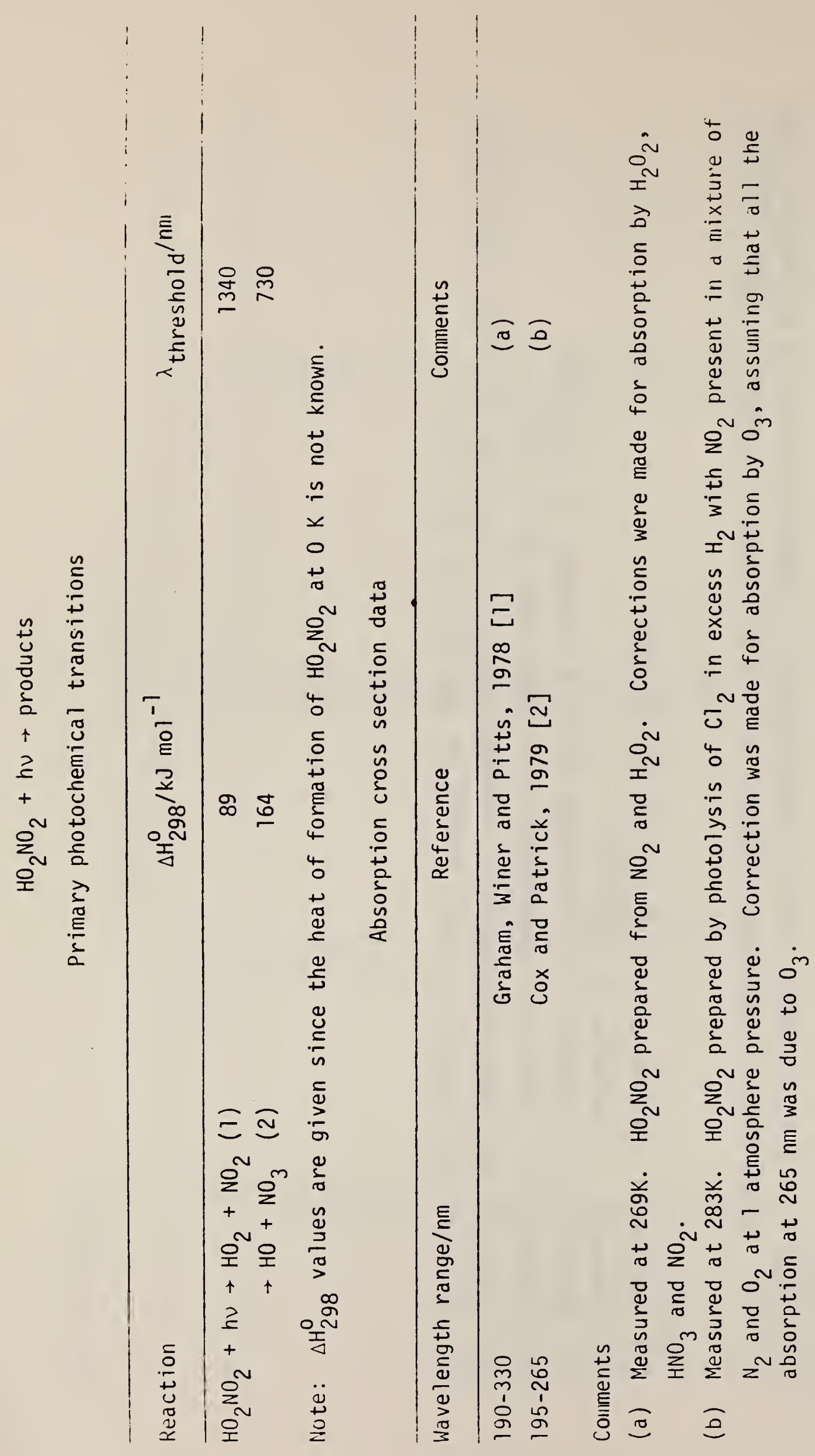




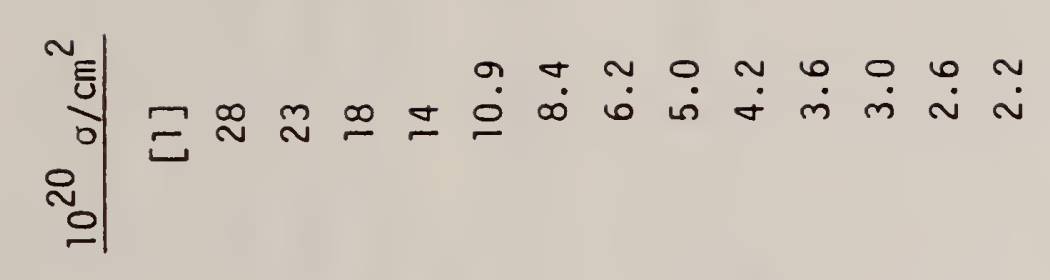

:

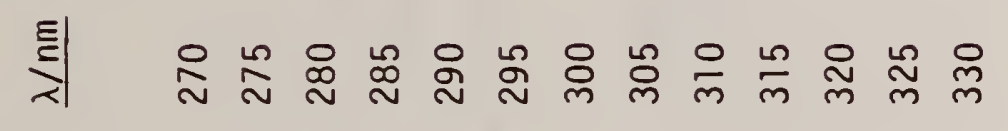

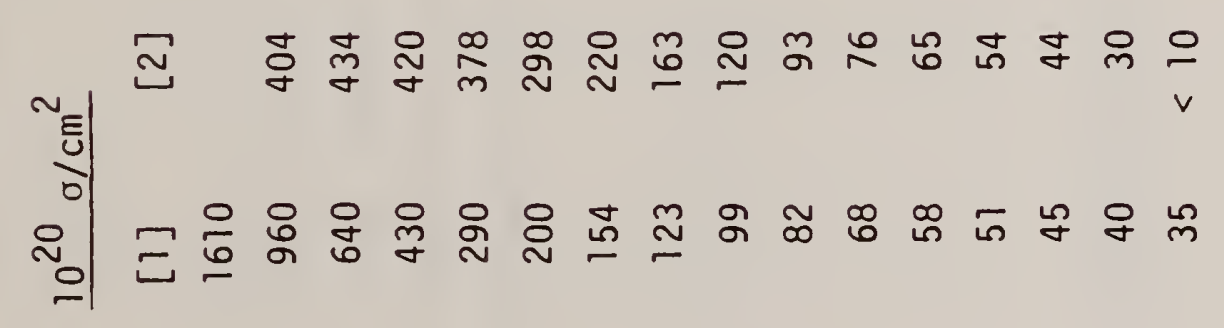

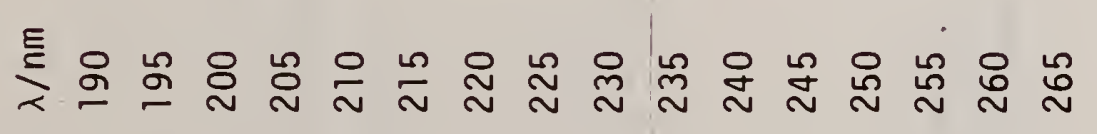




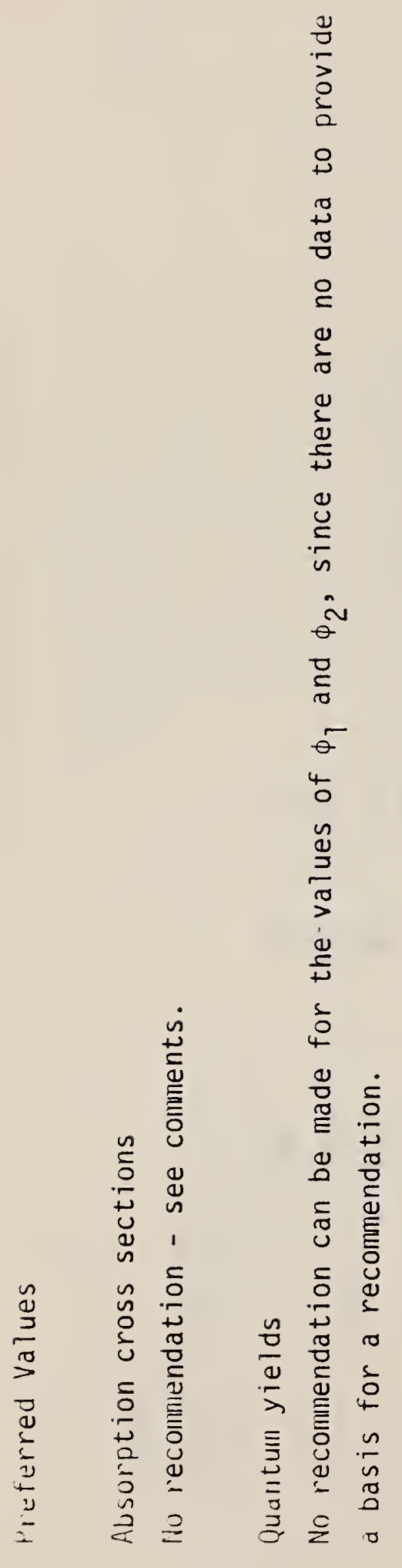




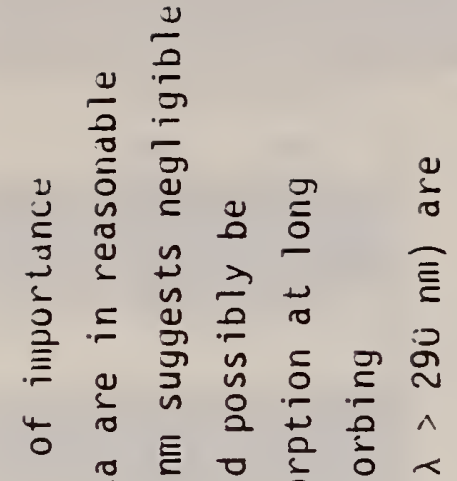

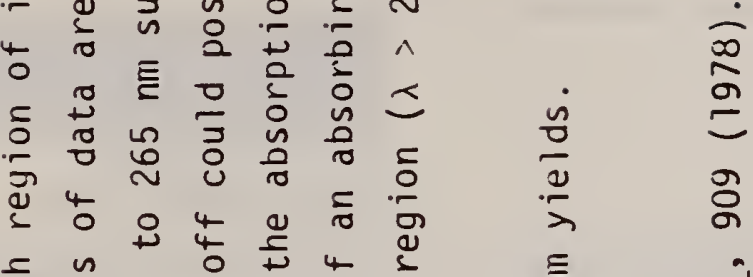

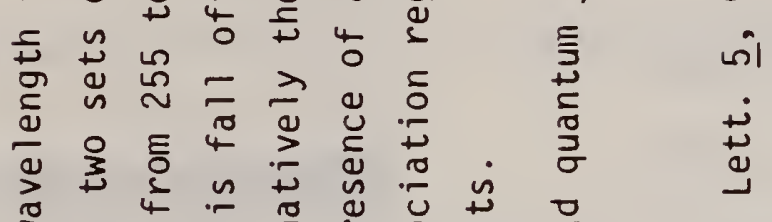

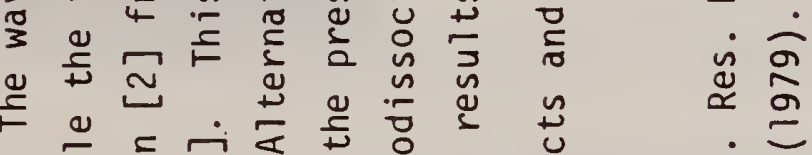

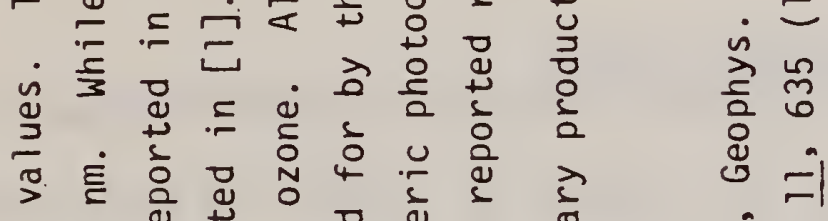

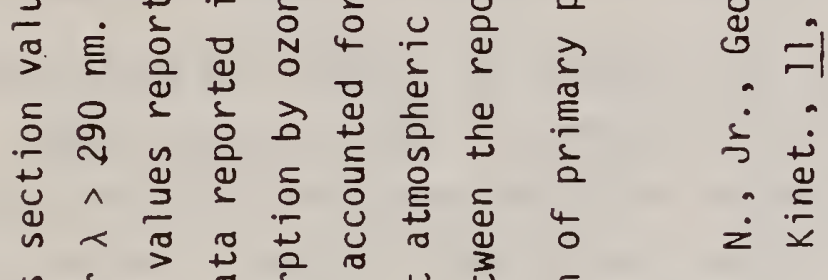

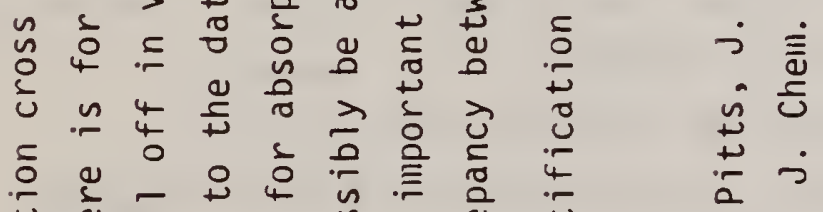

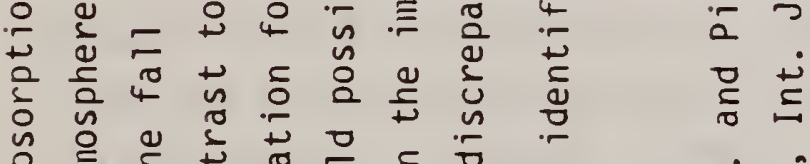

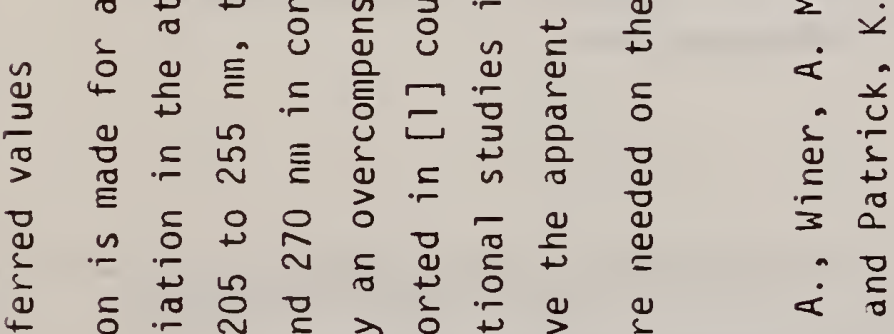

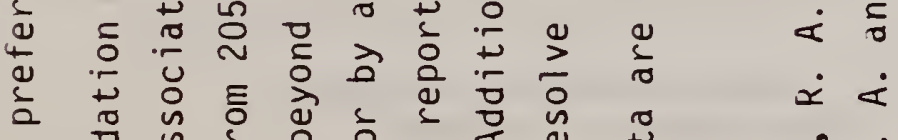

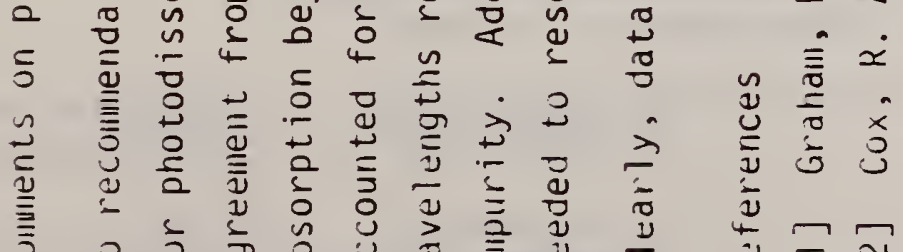

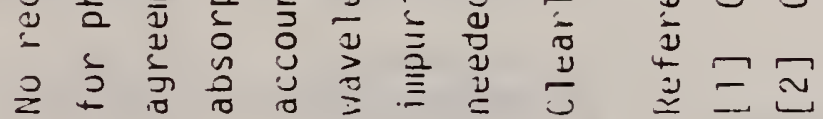




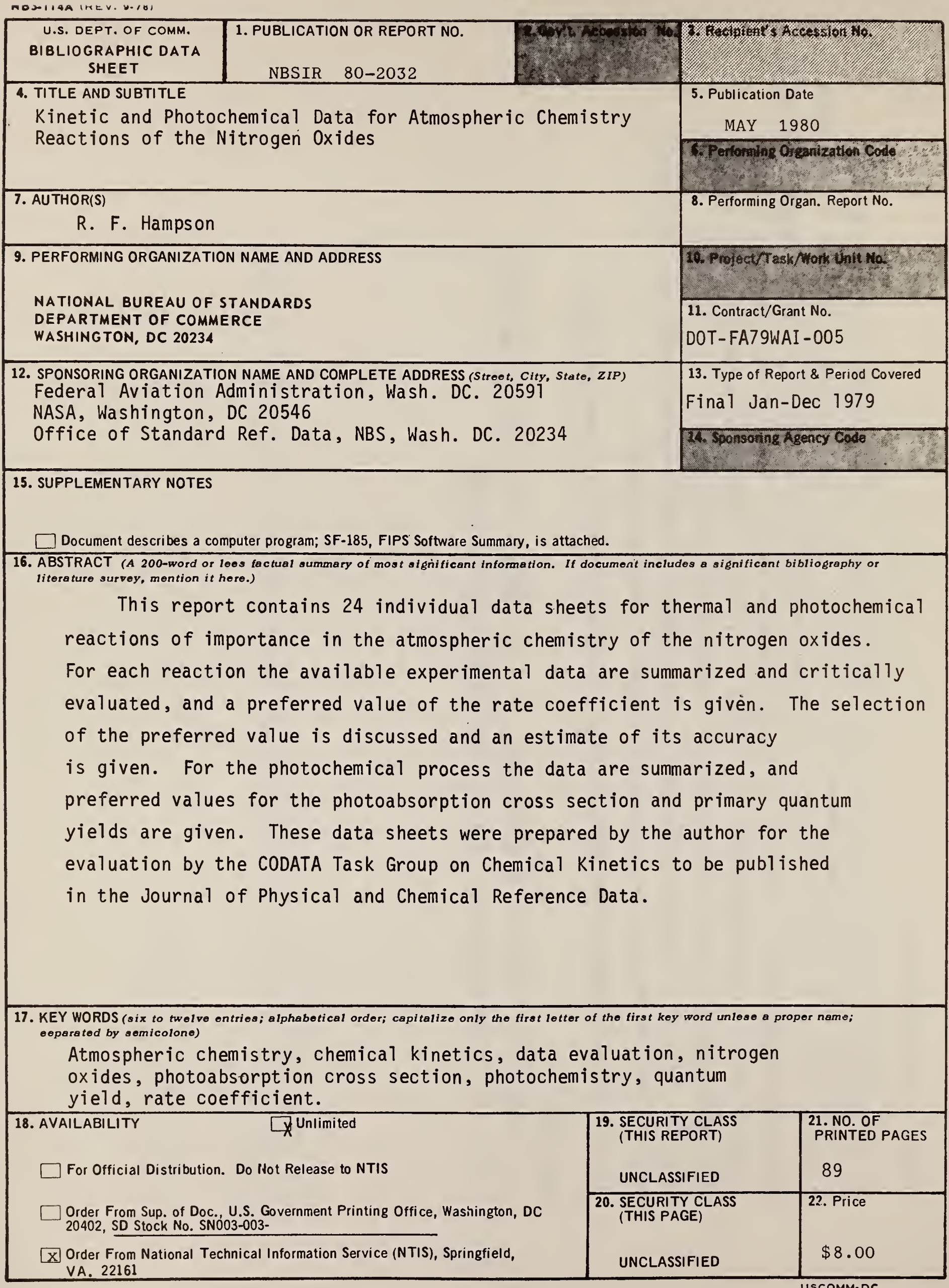





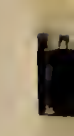

,

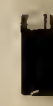

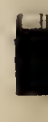

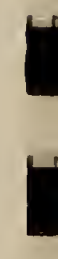

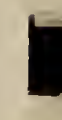

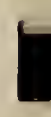

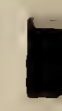

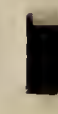

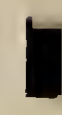

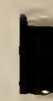

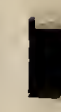

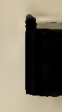

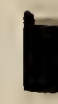

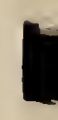

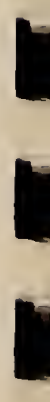

